\title{
Multi-component Tether Catalysis Synthesis of Highly Functionalized 4-(Pyridin-2-ylmethyl)-2-amino-pyrroles via Cascade Reaction is Accompanied by Decarboxylation
}

\author{
Kun Li, Li Chen, Yun-Xiang Fan, Yao Wei and Sheng-Jiao Yan* \\ Key Laboratory of Medicinal Chemistry for Natural Resources (Yunnan University), \\ Ministry of Education, School of Chemical Science and Technology, Yunnan \\ University, Kunming, 650091, P. R. China \\ *E-mail: yansj@ynu.edu.cn
}

\section{Supporting Information}

\section{Table of Contents}

Scheme S1. Alkylglyoxals were used as substrates to explore the reaction ..................................S4

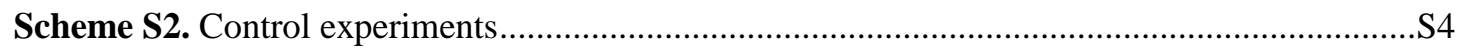

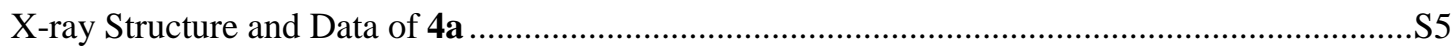

Figure S1. X-Ray crystal structure of $4 \mathrm{a}$, ellipsoids are drawn at the $30 \%$ probability level.........S5

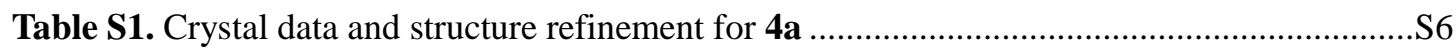

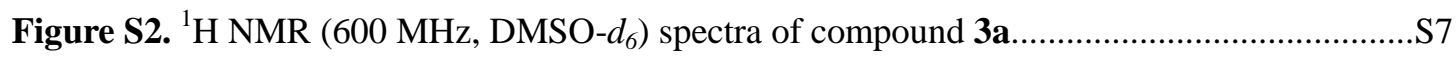

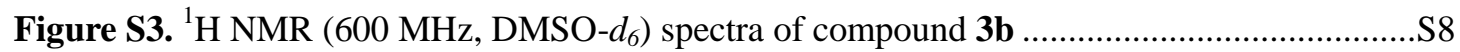

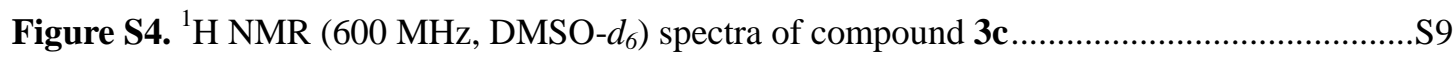

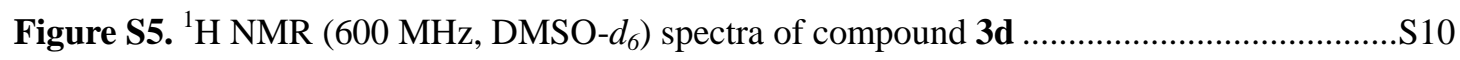

Figure S6. ${ }^{1} \mathrm{H}$ NMR $\left(600 \mathrm{MHz}\right.$, DMSO- $d_{6}$ ) spectra of compound 3e ........................................S11

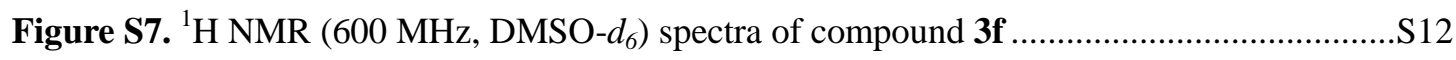

Figure S8. ${ }^{1} \mathrm{H}$ NMR $\left(600 \mathrm{MHz}, \mathrm{DMSO}-d_{6}\right)$ spectra of compound 3g........................................S13

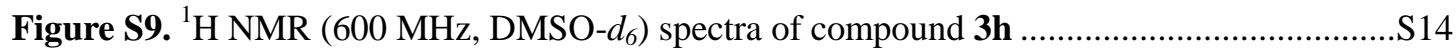

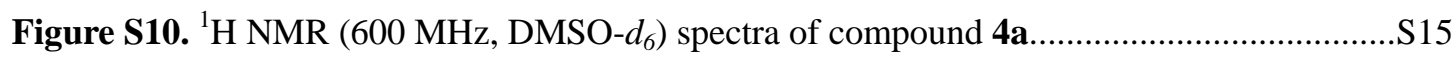

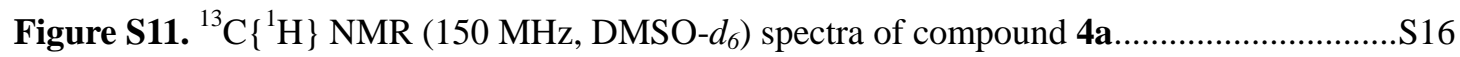

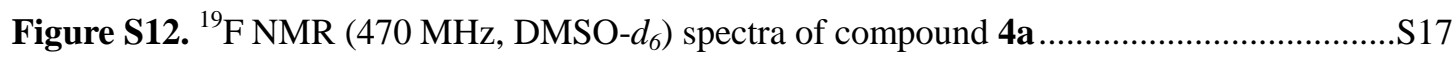

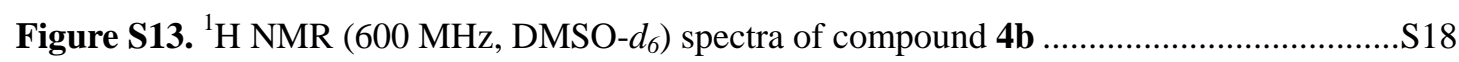

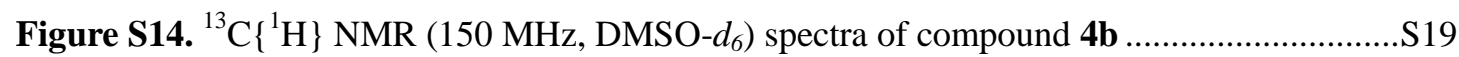

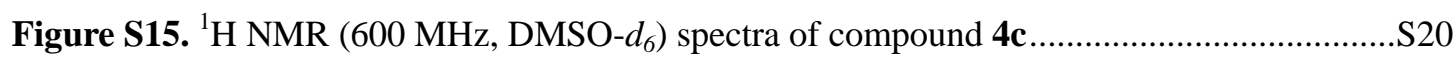




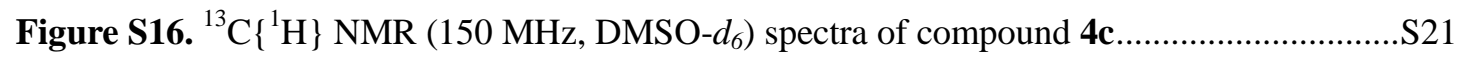

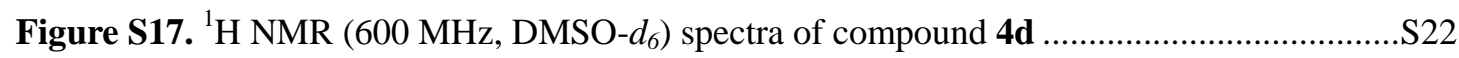

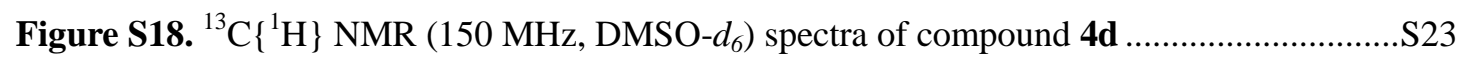

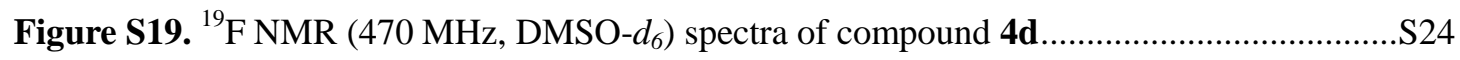

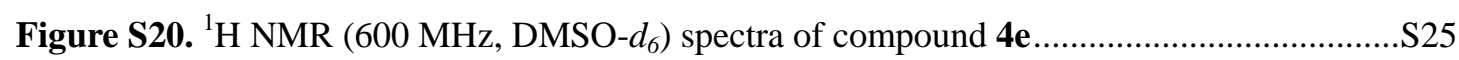

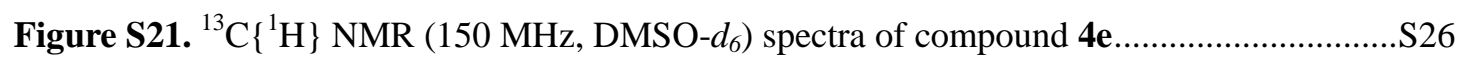

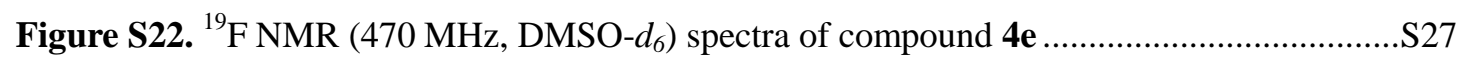

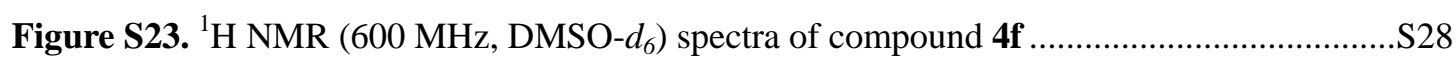

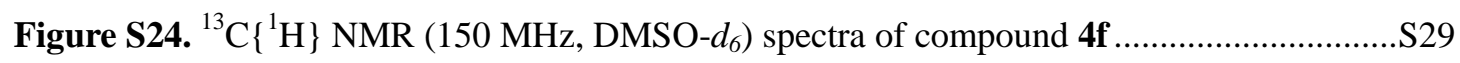

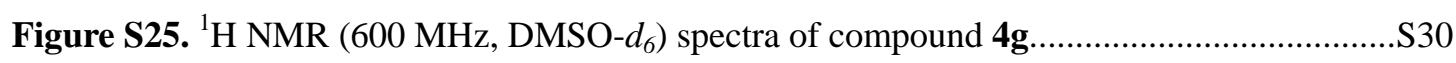

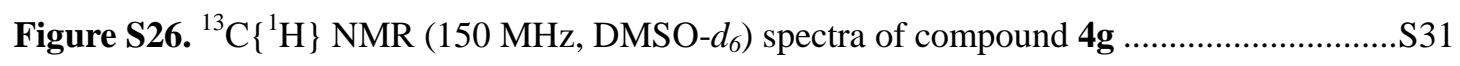

Figure S27. ${ }^{1} \mathrm{H}$ NMR (600 MHz, DMSO- $d_{6}$ ) spectra of compound $\mathbf{4 h}$.......................................S32

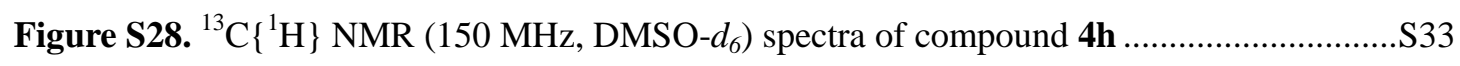

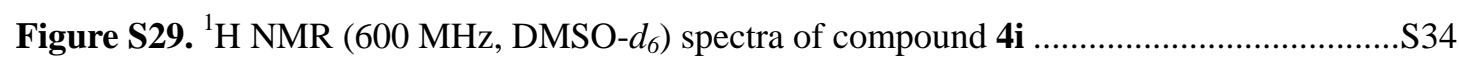

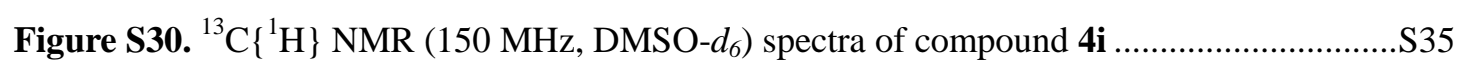

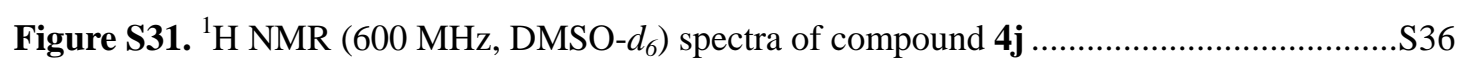

Figure S32. ${ }^{13} \mathrm{C}\left\{{ }^{1} \mathrm{H}\right\}$ NMR $\left(150 \mathrm{MHz}\right.$, DMSO- $\left.d_{6}\right)$ spectra of compound $\mathbf{4 j}$..............................S37

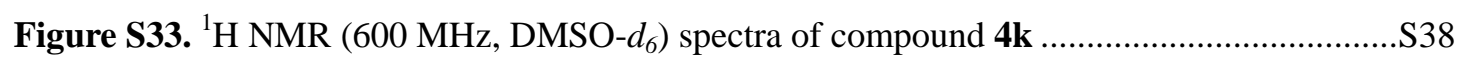

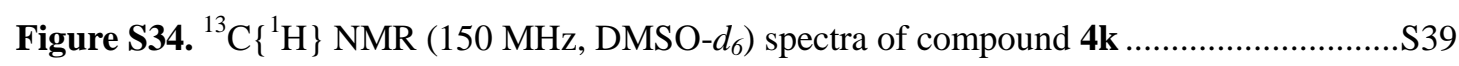

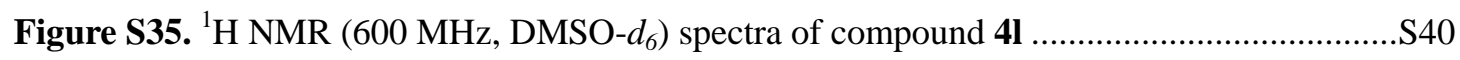

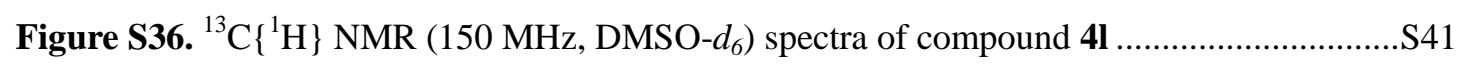

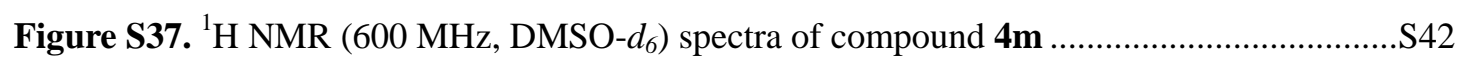

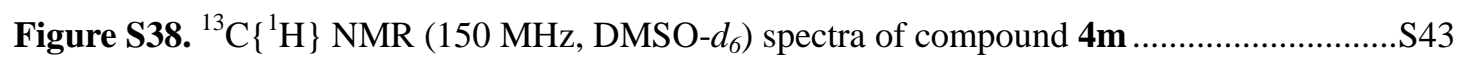

Figure S39. ${ }^{1} \mathrm{H}$ NMR (600 MHz, DMSO- $\left.d_{6}\right)$ spectra of compound $4 \mathbf{n}$......................................S44

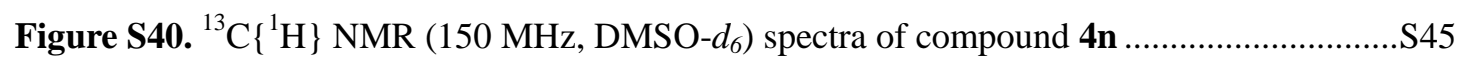

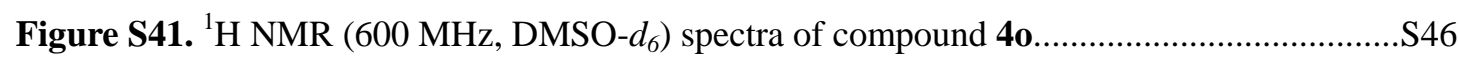

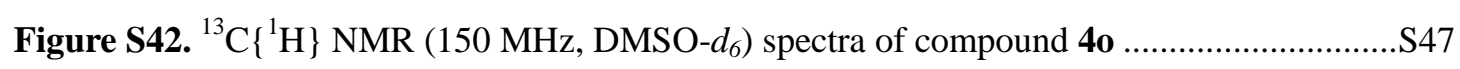

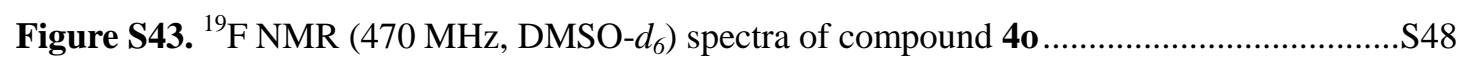

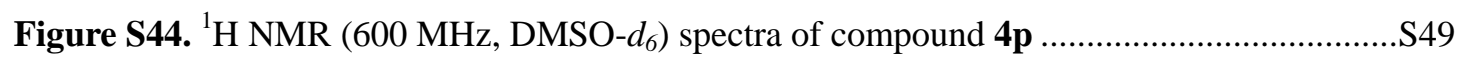

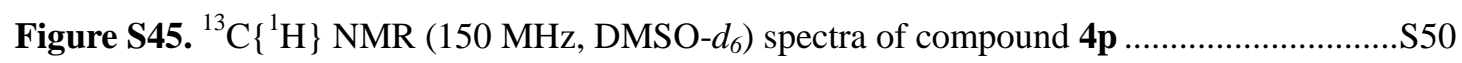

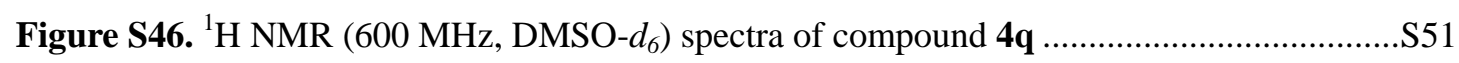

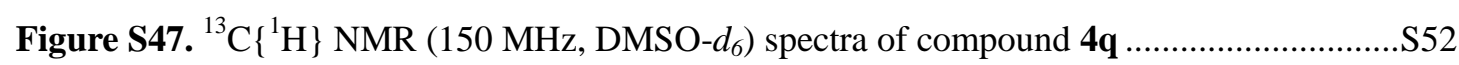

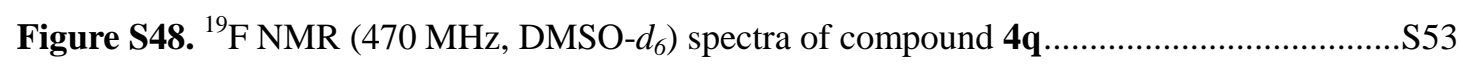

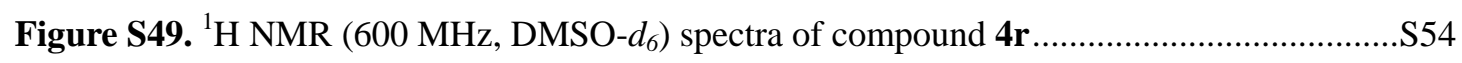

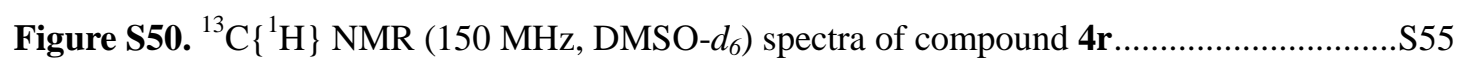

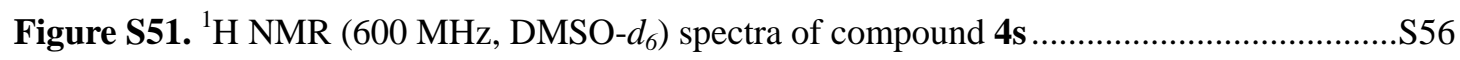

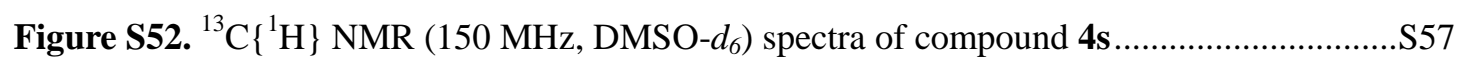

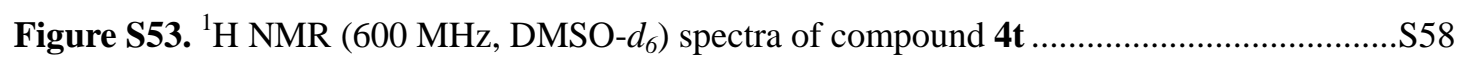

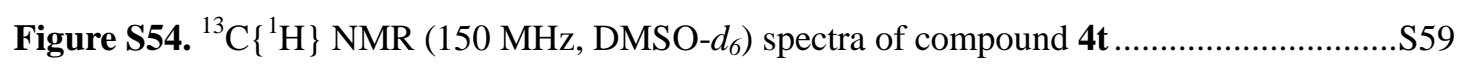

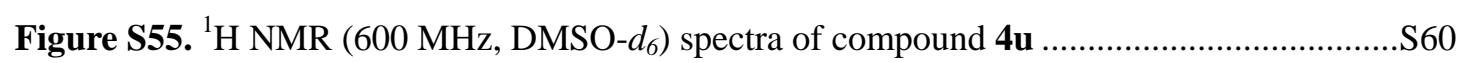




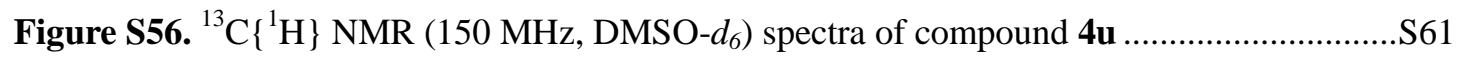

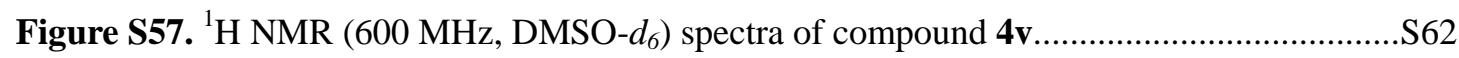

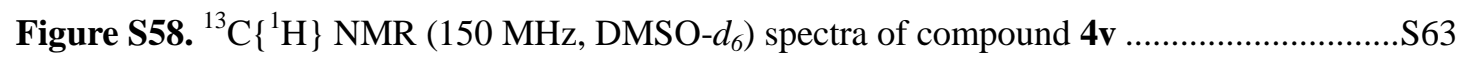

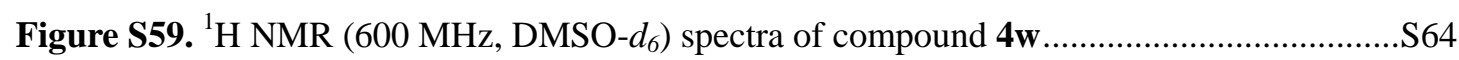

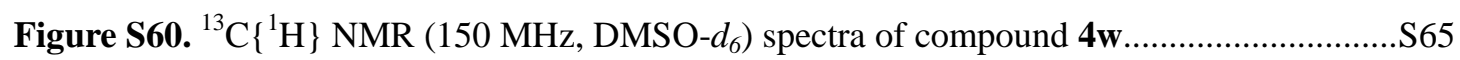

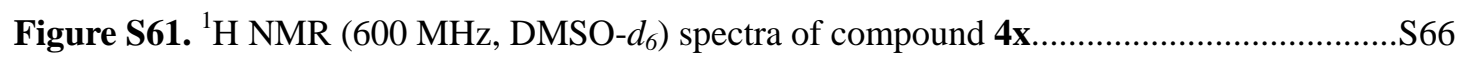

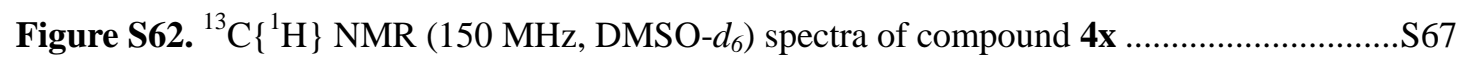

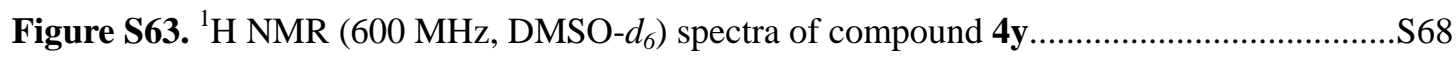

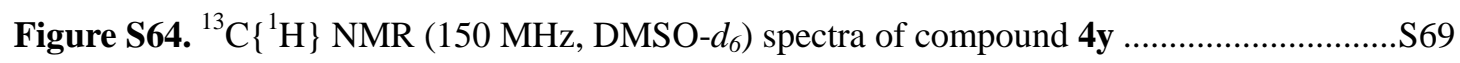

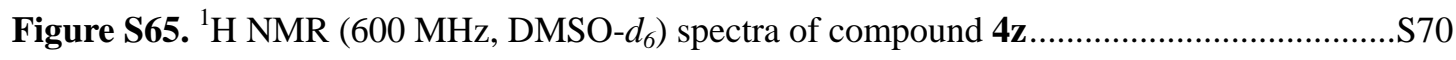

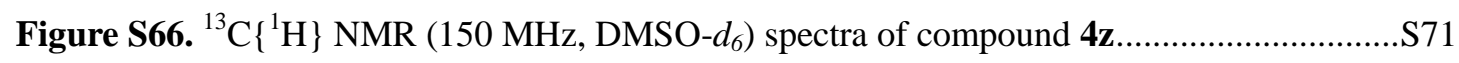

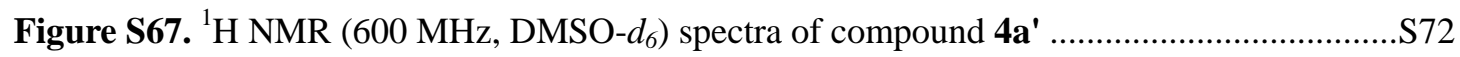

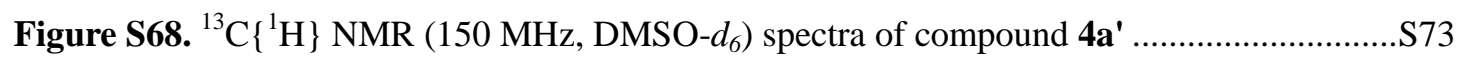

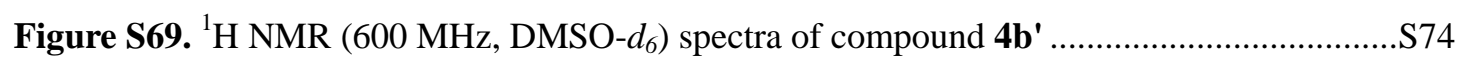

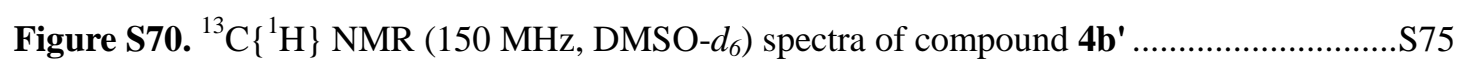

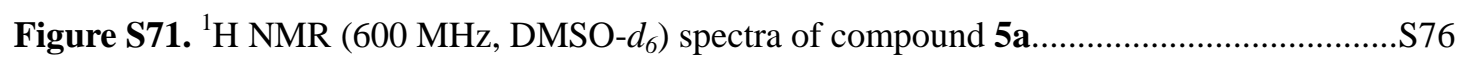

Figure S72. ${ }^{13} \mathrm{C}\left\{{ }^{1} \mathrm{H}\right\}$ NMR $\left(150 \mathrm{MHz}\right.$, DMSO- $\left.d_{6}\right)$ spectra of compound 5a .............................S77

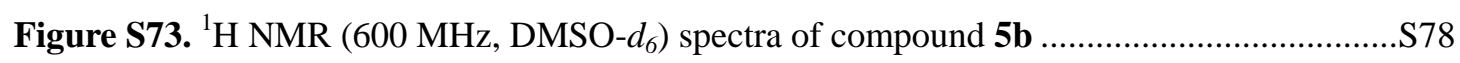

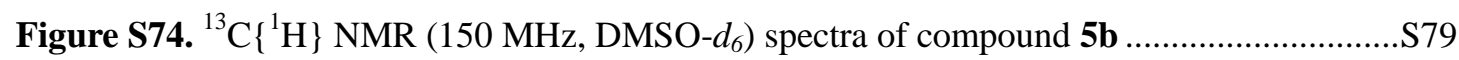

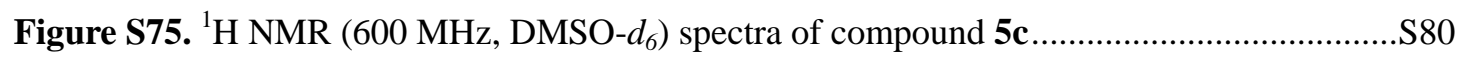

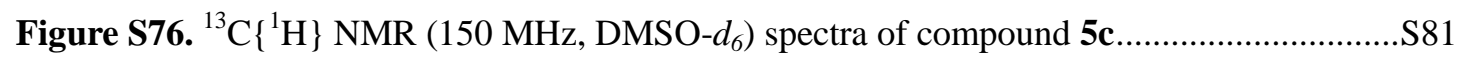

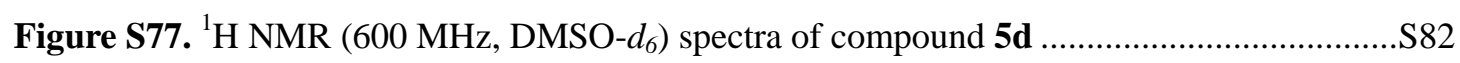

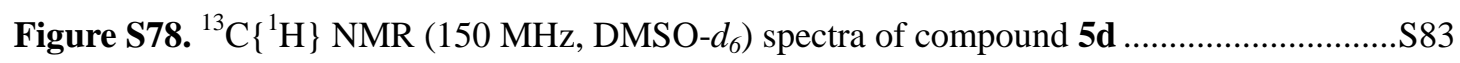

Figure S79. ${ }^{1} \mathrm{H}$ NMR (600 MHz, DMSO- $\left.d_{6}\right)$ spectra of compound 5e .......................................S8

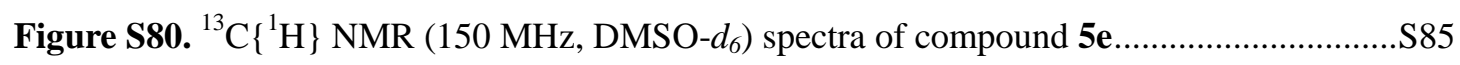

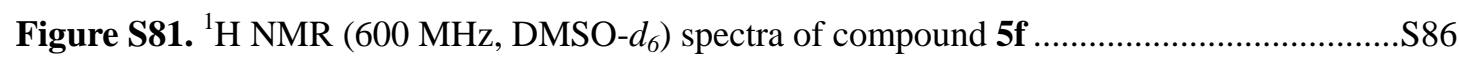

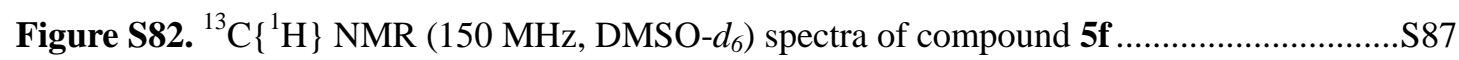

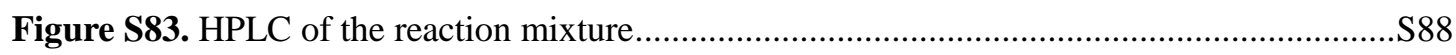

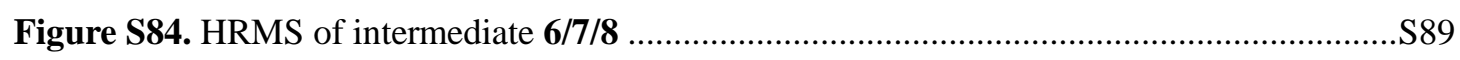

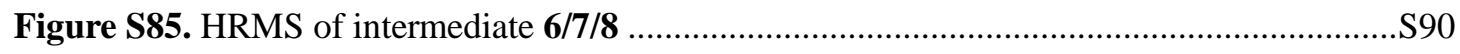

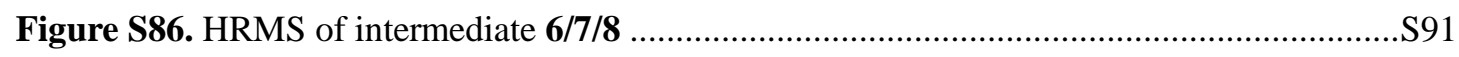

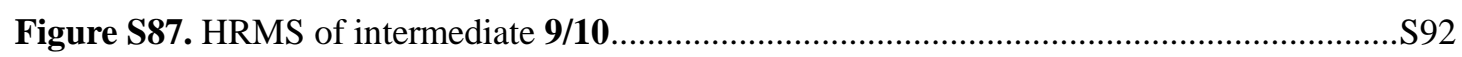

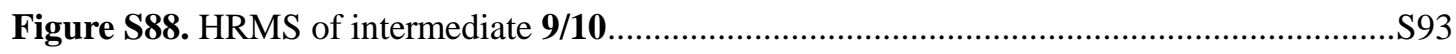

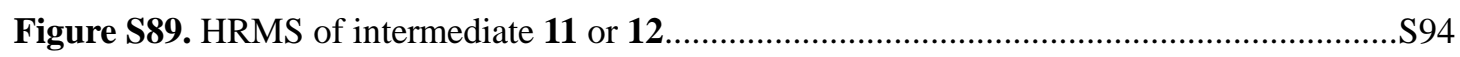

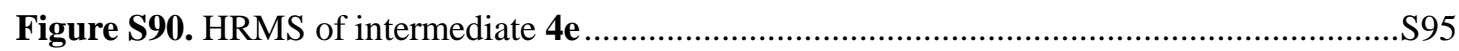

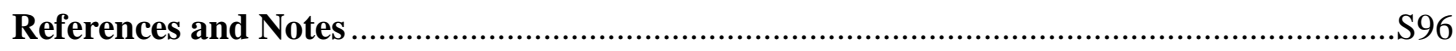


$\overbrace{\mathrm{N}}$<smiles>[R]C(=O)C(O)O</smiles>

2e: $\mathrm{R}=\mathrm{H}$

2f: $\mathrm{R}=\mathrm{Me}$

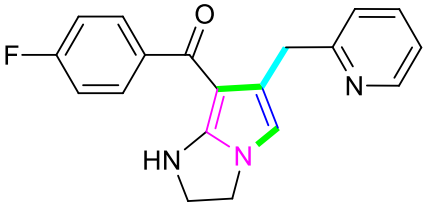

4c': yield=0\%

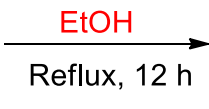

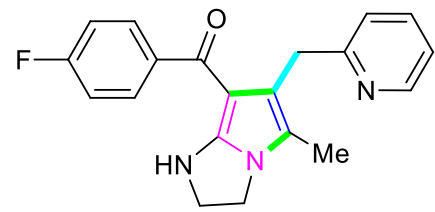

4d': yield=0\%

Scheme S1. Alkylglyoxals were used as substrates to explore the reaction<smiles>CCOC(=O)Cc1ccccc1</smiles>

1d: $Z=\mathrm{N}, \mathrm{Y}=\mathrm{CH}$

1e: $Z=\mathrm{CH}, \mathrm{Y}=\mathrm{N}$

1f: $Z=\mathrm{CH}, Y=\mathrm{CH}$ 1g: $Z=\mathrm{CH}, \mathrm{Y}=\mathrm{C}-\mathrm{NO}_{2}$

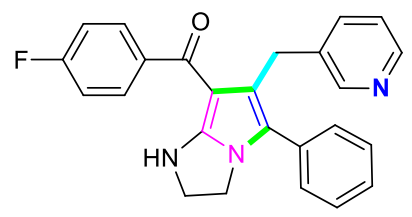

4d': yield $=0 \%$

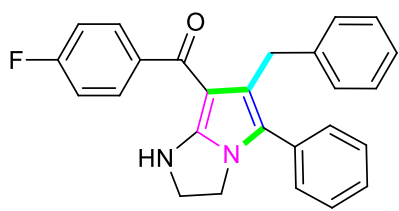

4f': yield $=0 \%$<smiles>O=C(C=C1NCCN1)c1ccc(F)cc1</smiles>

$\mathrm{EtOH}$ Reflux, $12 \mathrm{~h}$<smiles>O=C(c1ccccc1)C(O)O</smiles>
2b<smiles>[Y][X]c1[Z]cc(Cc2c(C(=O)c3ccc(F)cc3)c3n(c2-c2ccccc2)CCN3)cc1</smiles><smiles>O=C(c1ccc(F)cc1)c1c(Cc2ccncc2)c(-c2ccccc2)n2c1NCC2</smiles>

$4 e^{\prime}$ : yield $=0 \%$

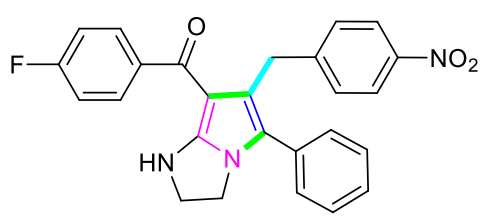

$\mathbf{4 g}^{\prime}$ : yield $=0 \%$

Scheme S2. Control experiments 


\section{$\underline{\text { X-ray Structure and Data of } 4 \mathbf{a}}$}

Single crystal culture and confirmation: First, compound $\mathbf{4 a}$ was added to a bottle and dissolved by the addition of ethyl acetate. Then, the bottle was placed in a jar, and petroleum ether $(15 \mathrm{~mL})$ was added to the bottle. The jar was sealed, and the bottle within the jar remained at room temperature for 6 days. Some crystals appeared, and for single crystal parsing, crystals were selected with sizes of $0.25 \mathrm{~mm} \times 0.23 \mathrm{~mm} \times$ $0.20 \mathrm{~mm}$. The APEX DUO diffractometer was used to obtain single crystal diffraction at $296.15 \mathrm{~K}$ with the use of graphite monochromatic Mo K (lambda $=0.71073 \mathrm{~A}$ ) for diffraction intensity data collection, using $\Phi$ and omega scanning. Multi-scan correction was performed for all intensity data. The crystal structure was solved by the atomic method using the SHELXT program (Supporting Information, Figure S1, CCDC 1920258) $)^{1}$.

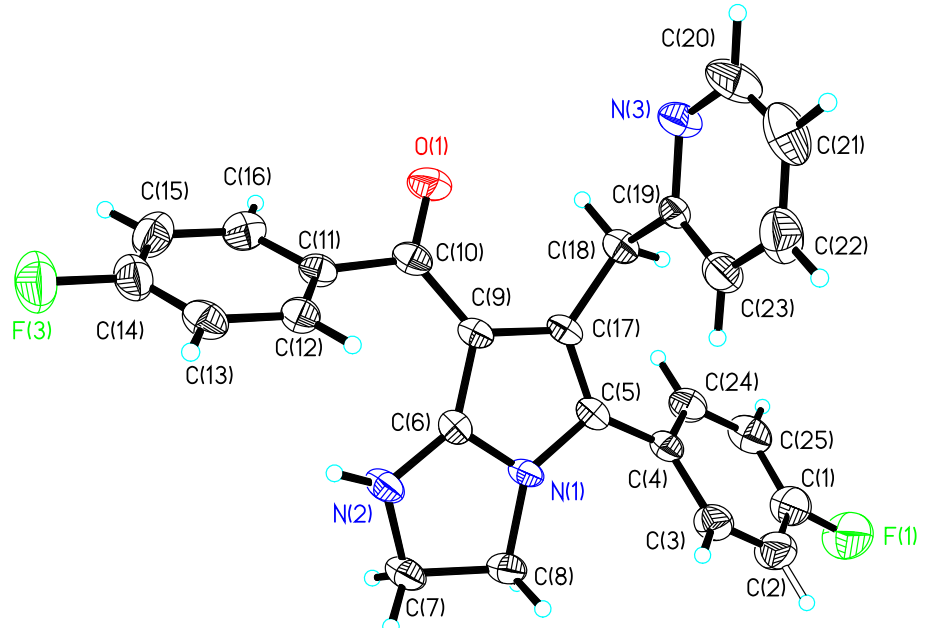

Figure S1. X-Ray crystal structure of $\mathbf{4 a}$, ellipsoids are drawn at the $30 \%$ probability level. 
Table S1. Crystal data and structure refinement for $\mathbf{4 a}$

\begin{tabular}{|c|c|}
\hline Identification code & 181116ysj_0m \\
\hline Empirical formula & C25 H18 F3 N3 O \\
\hline Formula weight & 433.42 \\
\hline Temperature & $296.15 \mathrm{~K}$ \\
\hline Wavelength & $0.71073 \AA$ \\
\hline Crystal system & Orthorhombic \\
\hline Space group & Pbca \\
\hline \multirow[t]{3}{*}{ Unit cell dimensions } & $\mathrm{a}=15.480(5) \AA$ \\
\hline & $\mathrm{b}=11.486(4) \AA$ \\
\hline & $\mathrm{c}=23.094(8) \AA$ \\
\hline Volume & $4106(2) \AA^{3}$ \\
\hline $\mathrm{Z}$ & 8 \\
\hline Density (calculated) & $1.402 \mathrm{Mg} / \mathrm{m}^{3}$ \\
\hline Absorption coefficient & $0.106 \mathrm{~mm}^{-1}$ \\
\hline $\mathrm{F}(000)$ & 1792 \\
\hline Crystal size & $0.25 \times 0.23 \times 0.20 \mathrm{~mm}^{3}$ \\
\hline Theta range for data collection & 2.378 to $24.996^{\circ}$. \\
\hline Index ranges & $-17<=\mathrm{h}<=18,-13<=\mathrm{k}<=13,-11<=1<=27$ \\
\hline Reflections collected & 16108 \\
\hline Independent reflections & $3609[\mathrm{R}($ int $)=0.1718]$ \\
\hline Completeness to theta $=24.996^{\circ}$ & $99.9 \%$ \\
\hline Absorption correction & Semi-empirical from equivalents \\
\hline Max. and min. transmission & 0.7456 and 0.6374 \\
\hline Refinement method & Full-matrix least-squares on $\mathrm{F}^{2}$ \\
\hline Data / restraints / parameters & $3609 / 0 / 300$ \\
\hline Goodness-of-fit on $\mathrm{F}^{2}$ & 0.997 \\
\hline Final $R$ indices $[I>2 \operatorname{sigma}(I)]$ & $\mathrm{R} 1=0.0657, \mathrm{wR} 2=0.1057$ \\
\hline $\mathrm{R}$ indices (all data) & $\mathrm{R} 1=0.2030, \mathrm{wR} 2=0.1532$ \\
\hline Extinction coefficient & $0.0031(4)$ \\
\hline Largest diff. peak and hole & 0.191 and -0.208 e. $\AA^{-3}$ \\
\hline
\end{tabular}




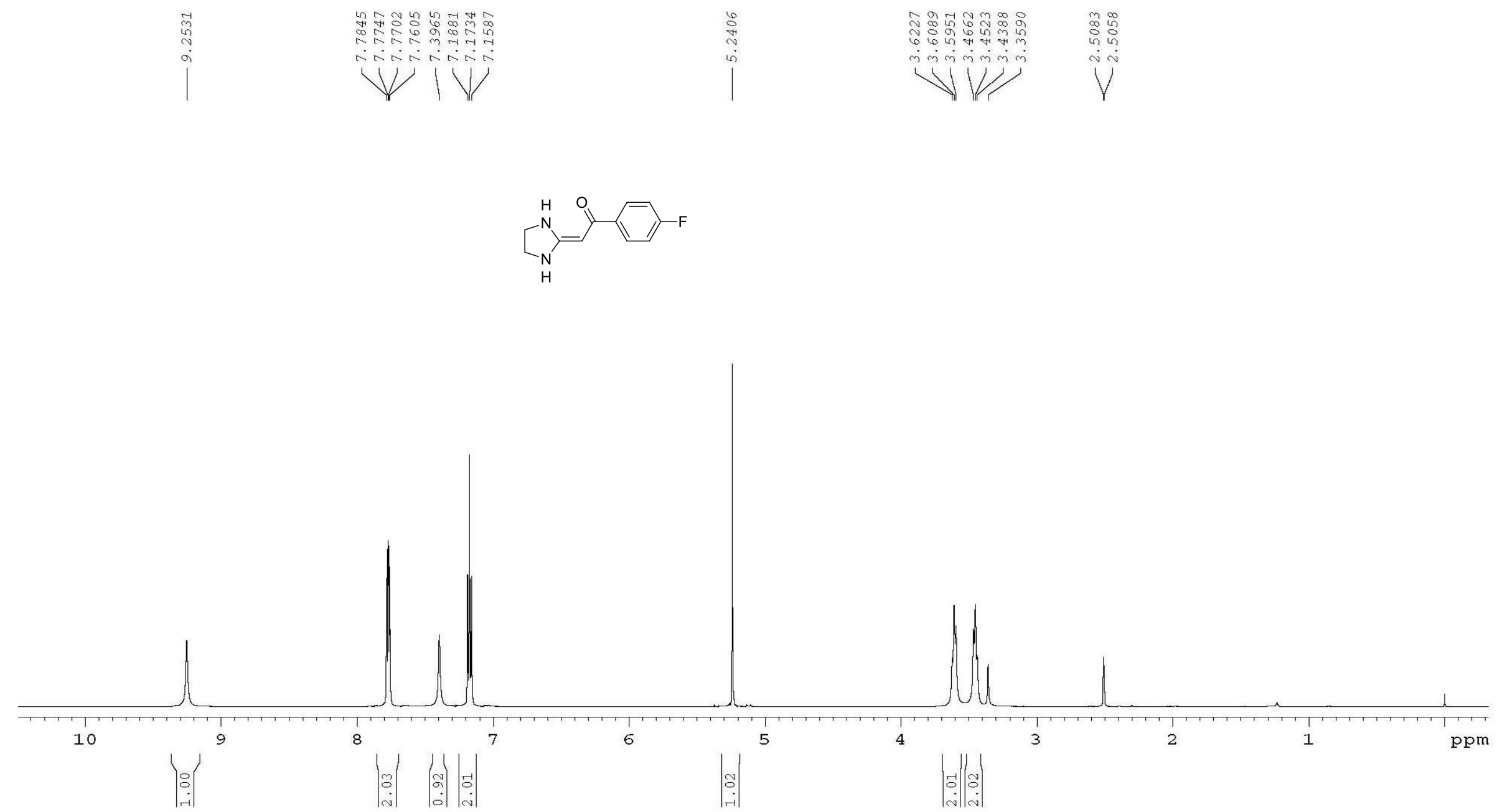

Figure S2. ${ }^{1} \mathrm{H}$ NMR $\left(600 \mathrm{MHz}, \mathrm{DMSO}-d_{6}\right)$ spectra of compound 3a 


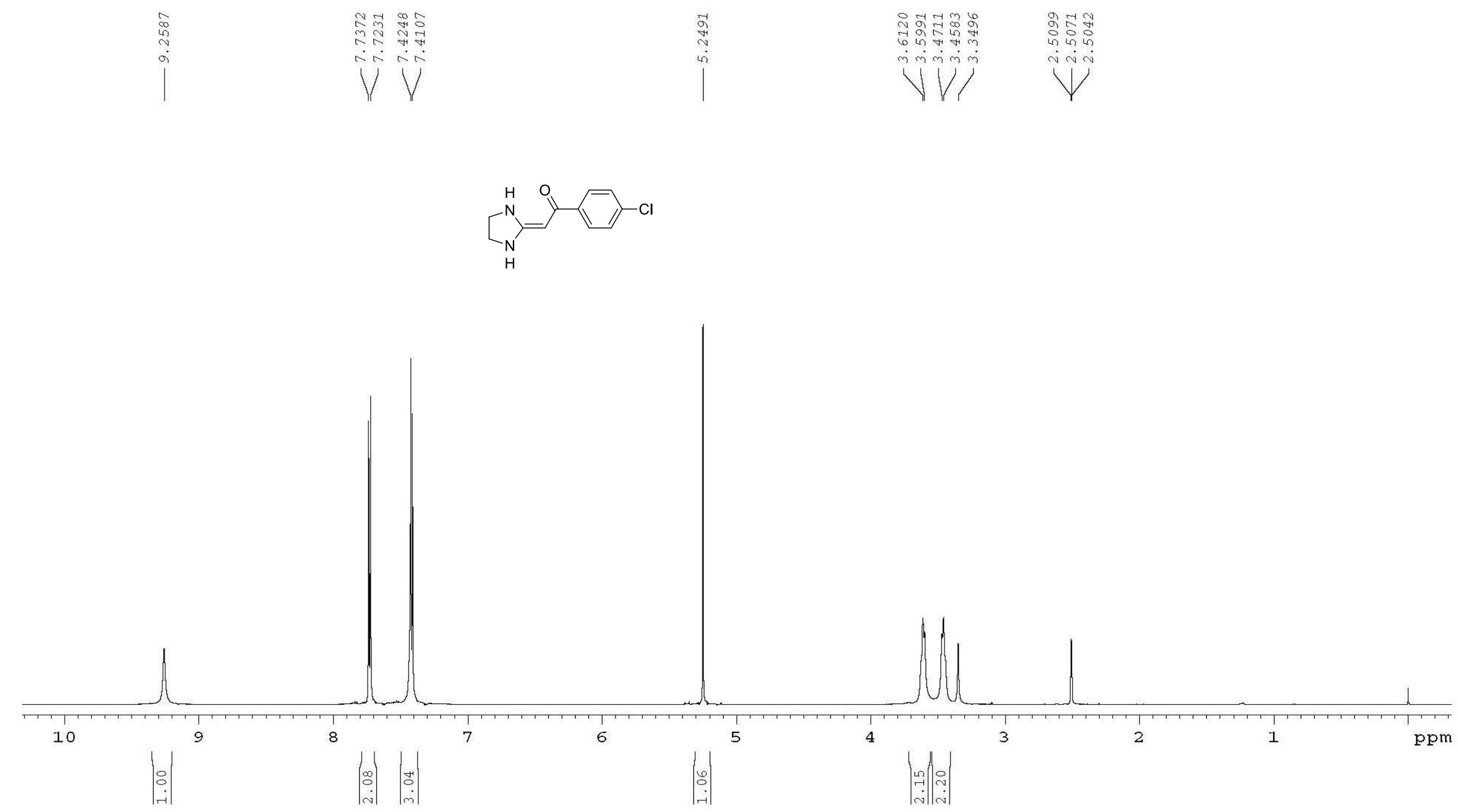

Figure S3. ${ }^{1} \mathrm{H}$ NMR $\left(600 \mathrm{MHz}, \mathrm{DMSO}-d_{6}\right)$ spectra of compound 3b 


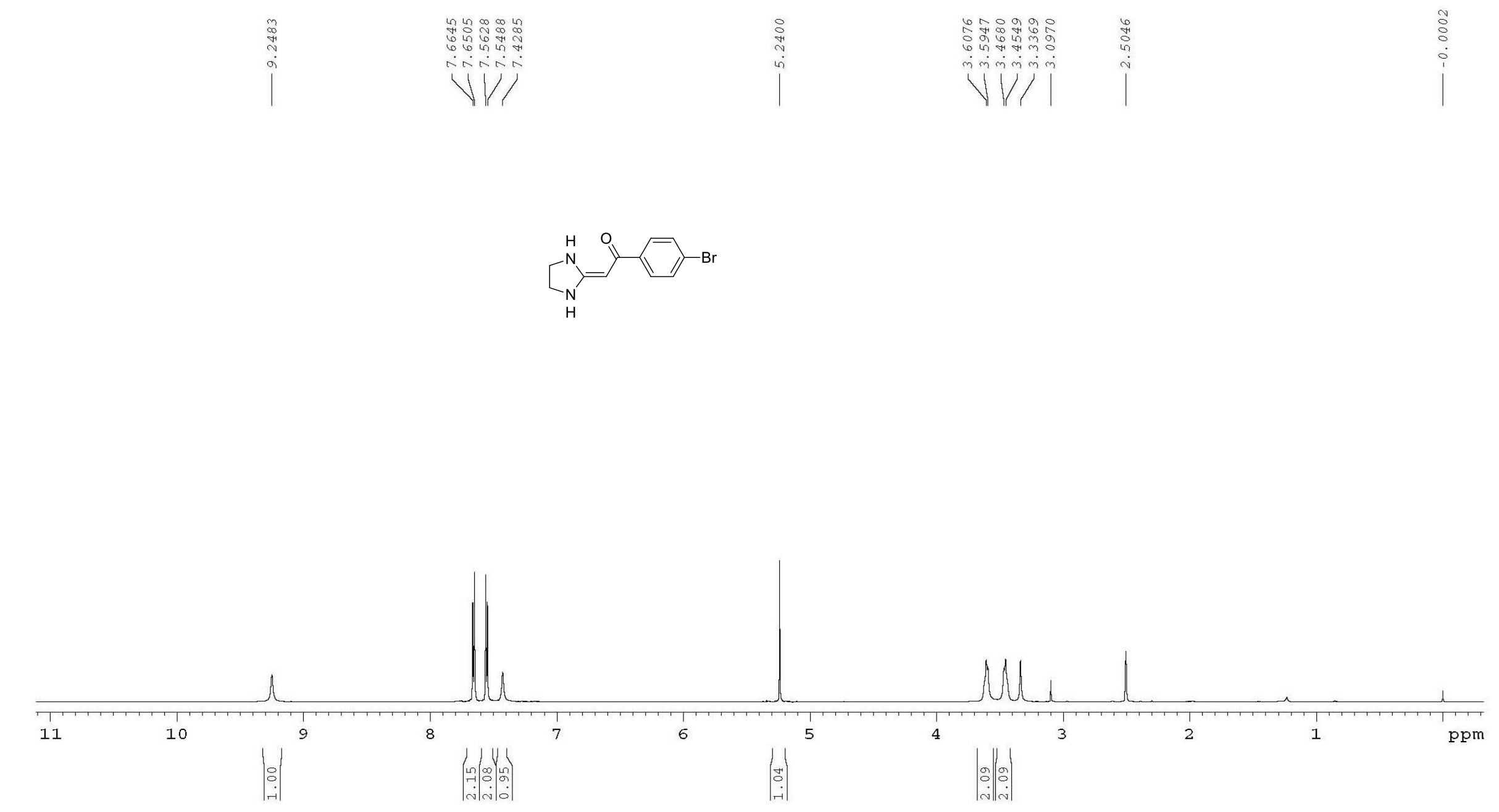

Figure S4. ${ }^{1} \mathrm{H}$ NMR $\left(600 \mathrm{MHz}, \mathrm{DMSO}-d_{6}\right)$ spectra of compound $\mathbf{3 c}$ 

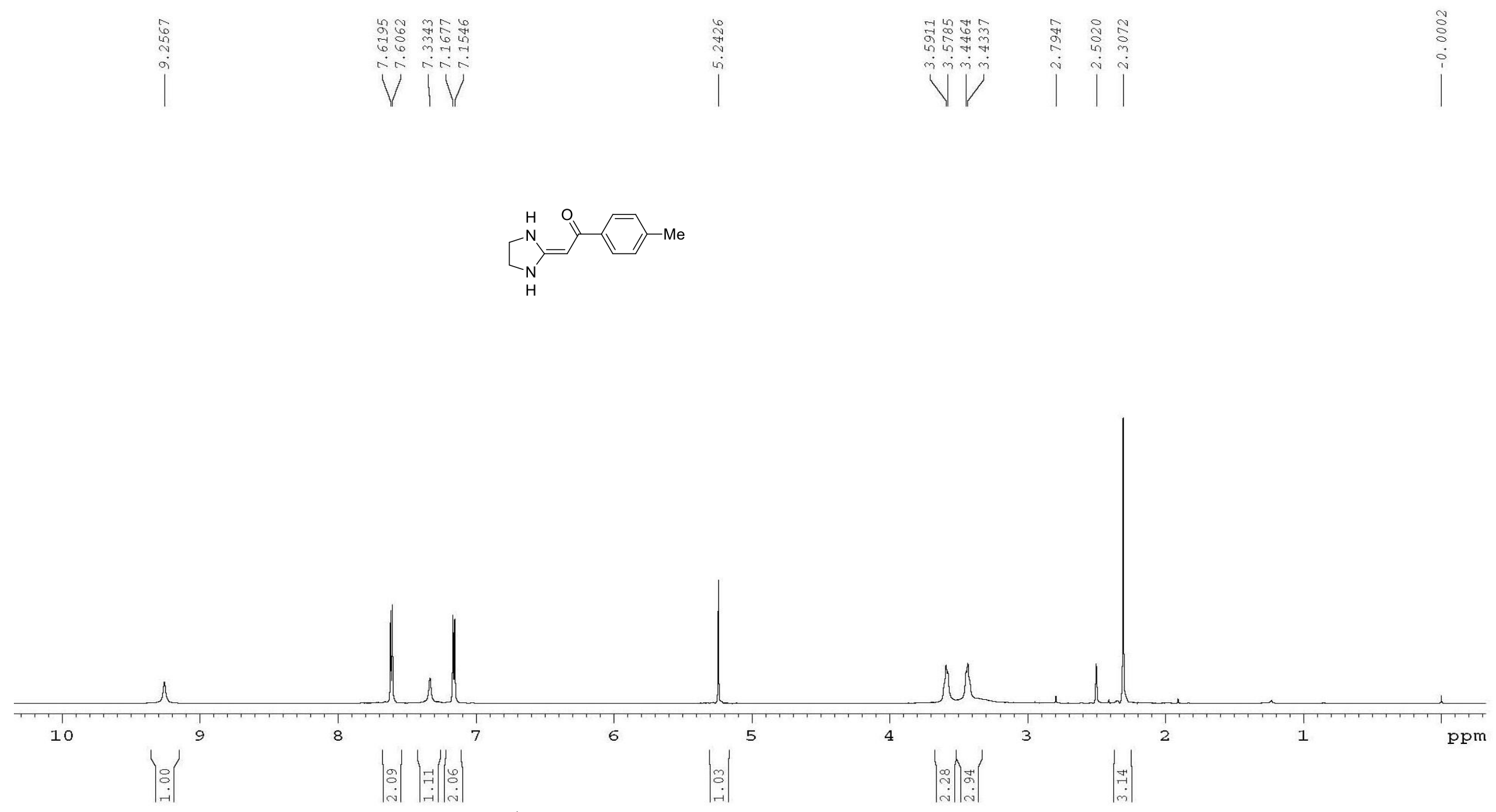

Figure S5. ${ }^{1} \mathrm{H}$ NMR $\left(600 \mathrm{MHz}, \mathrm{DMSO}-d_{6}\right)$ spectra of compound 3d 


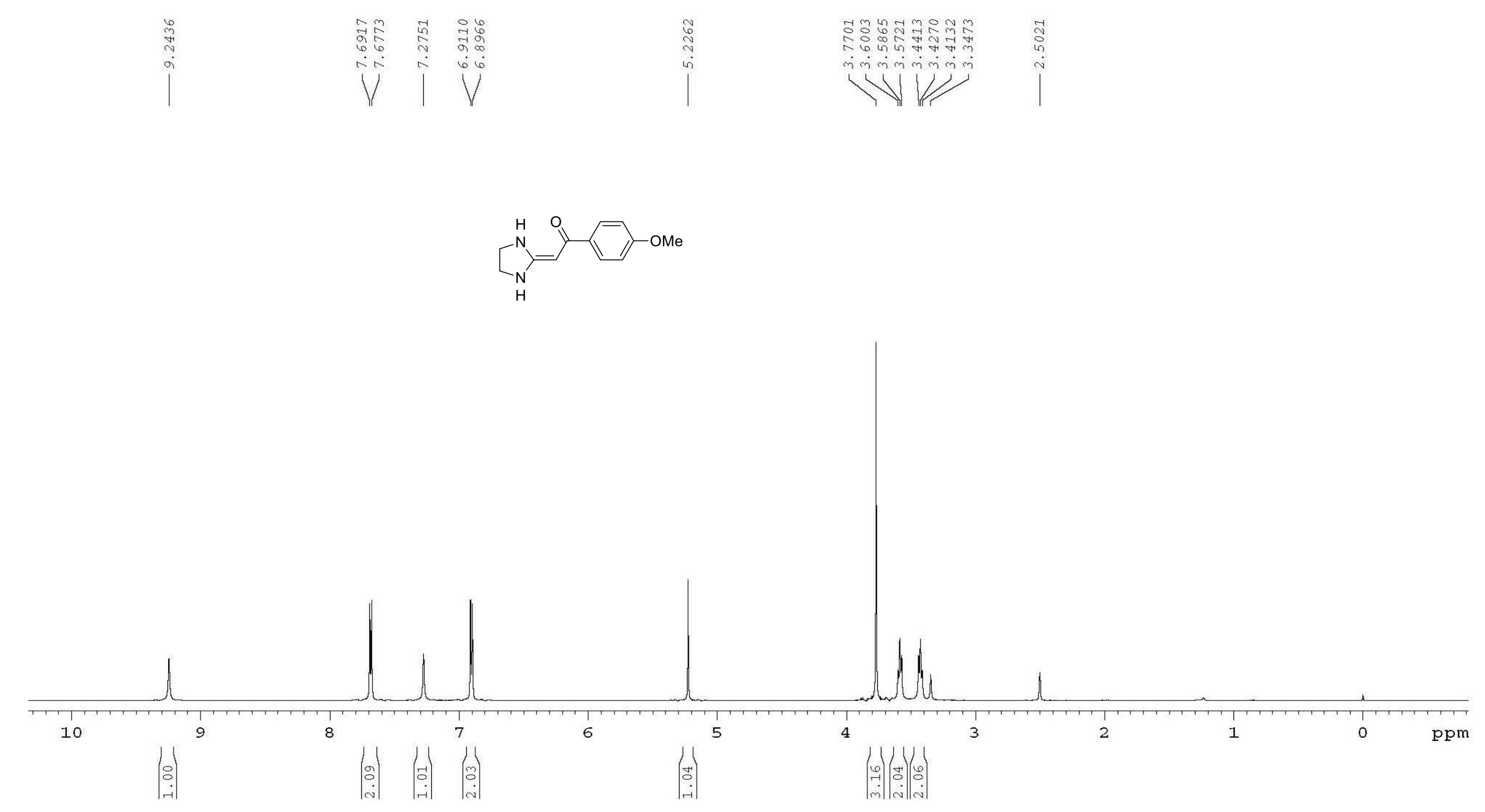

Figure S6. ${ }^{1} \mathrm{H}$ NMR $\left(600 \mathrm{MHz}, \mathrm{DMSO}-d_{6}\right)$ spectra of compound $\mathbf{3 e}$ 

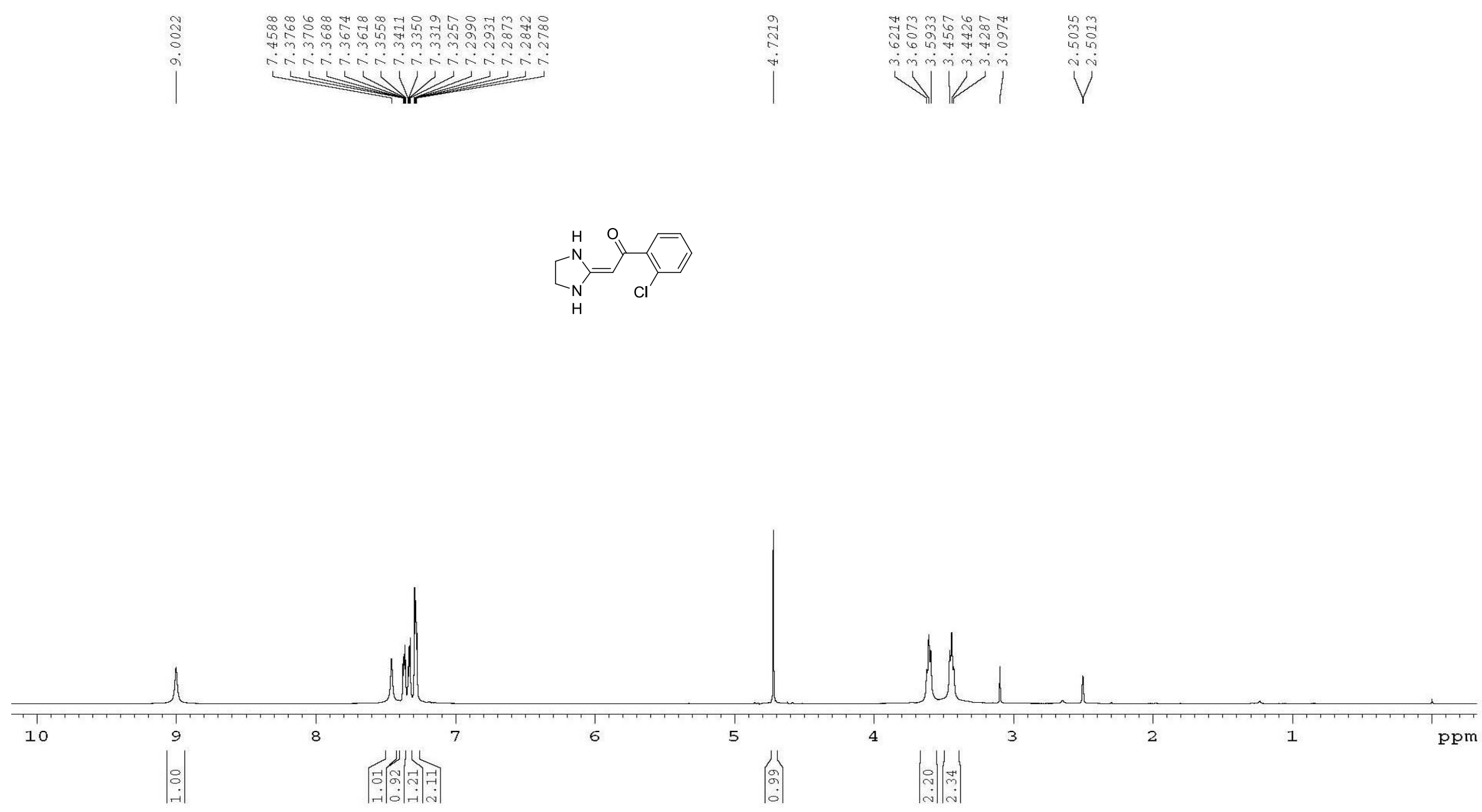

Figure S7. ${ }^{1} \mathrm{H}$ NMR $\left(600 \mathrm{MHz}, \mathrm{DMSO}-d_{6}\right)$ spectra of compound $\mathbf{3 f}$ 
HKULIUN DIVISO

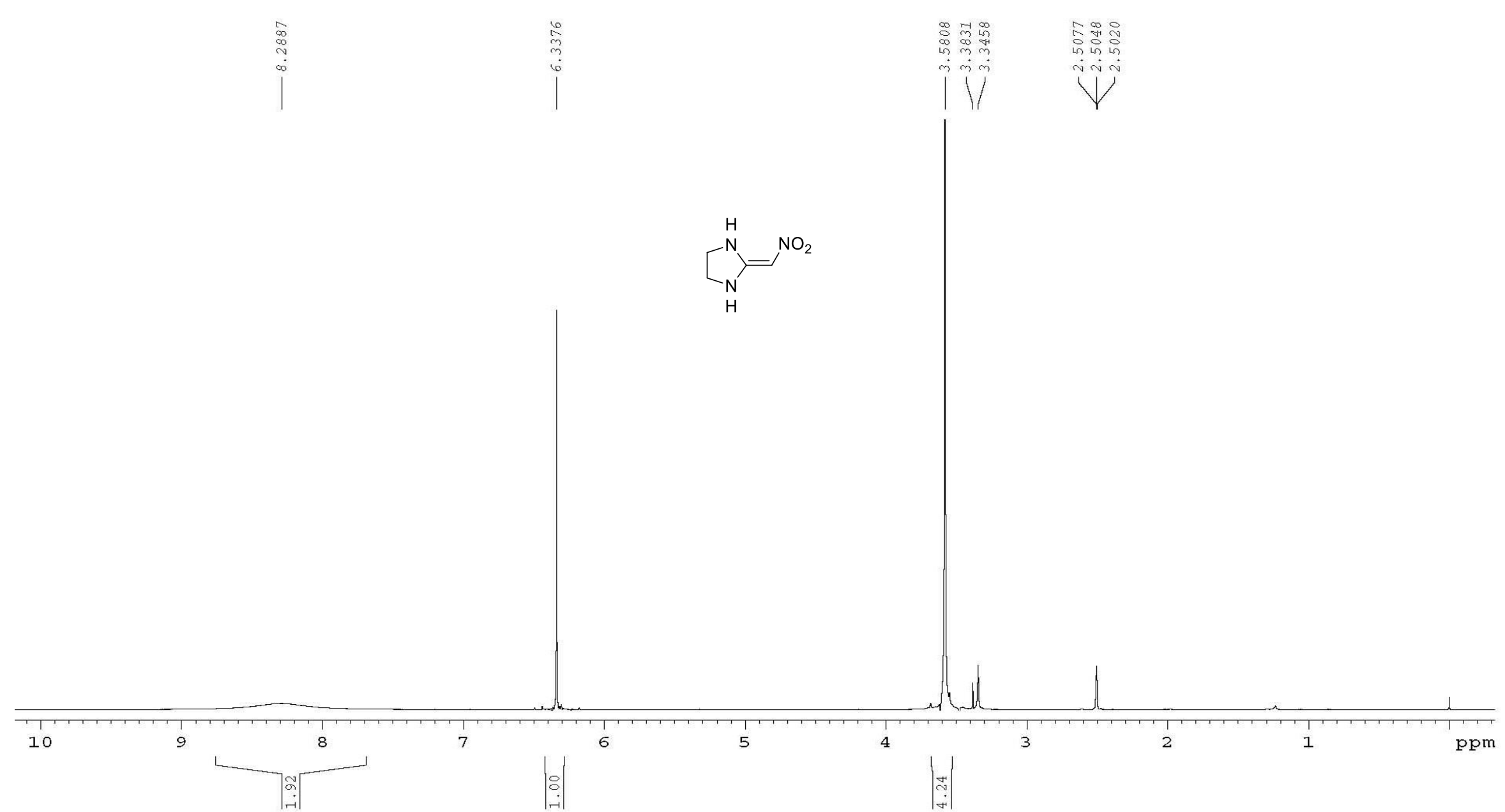

Figure S8. ${ }^{1} \mathrm{H}$ NMR $\left(600 \mathrm{MHz}, \mathrm{DMSO}-d_{6}\right)$ spectra of compound $\mathbf{3 g}$ 


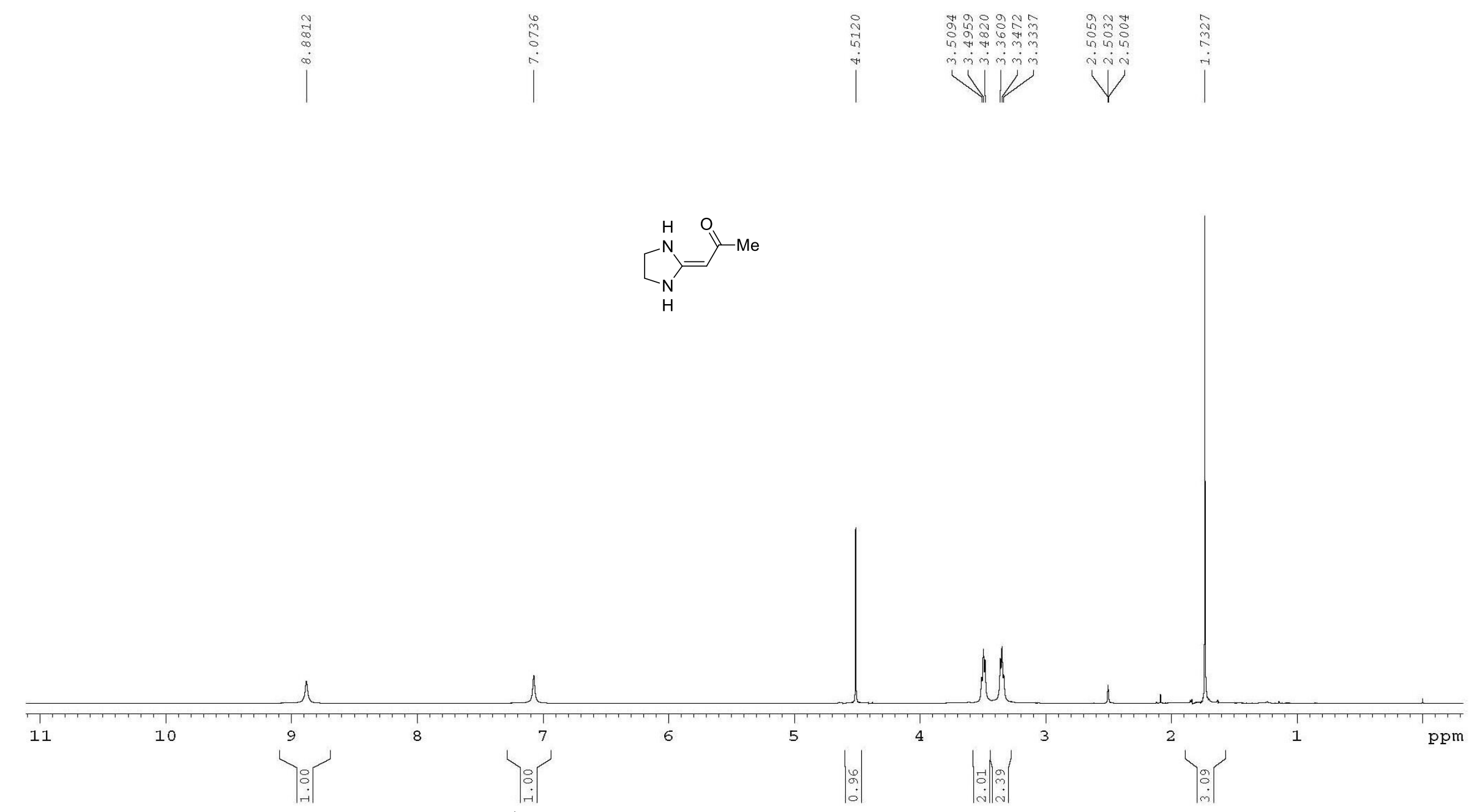

Figure S9. ${ }^{1} \mathrm{H}$ NMR $\left(600 \mathrm{MHz}, \mathrm{DMSO}-d_{6}\right)$ spectra of compound $\mathbf{3 h}$ 


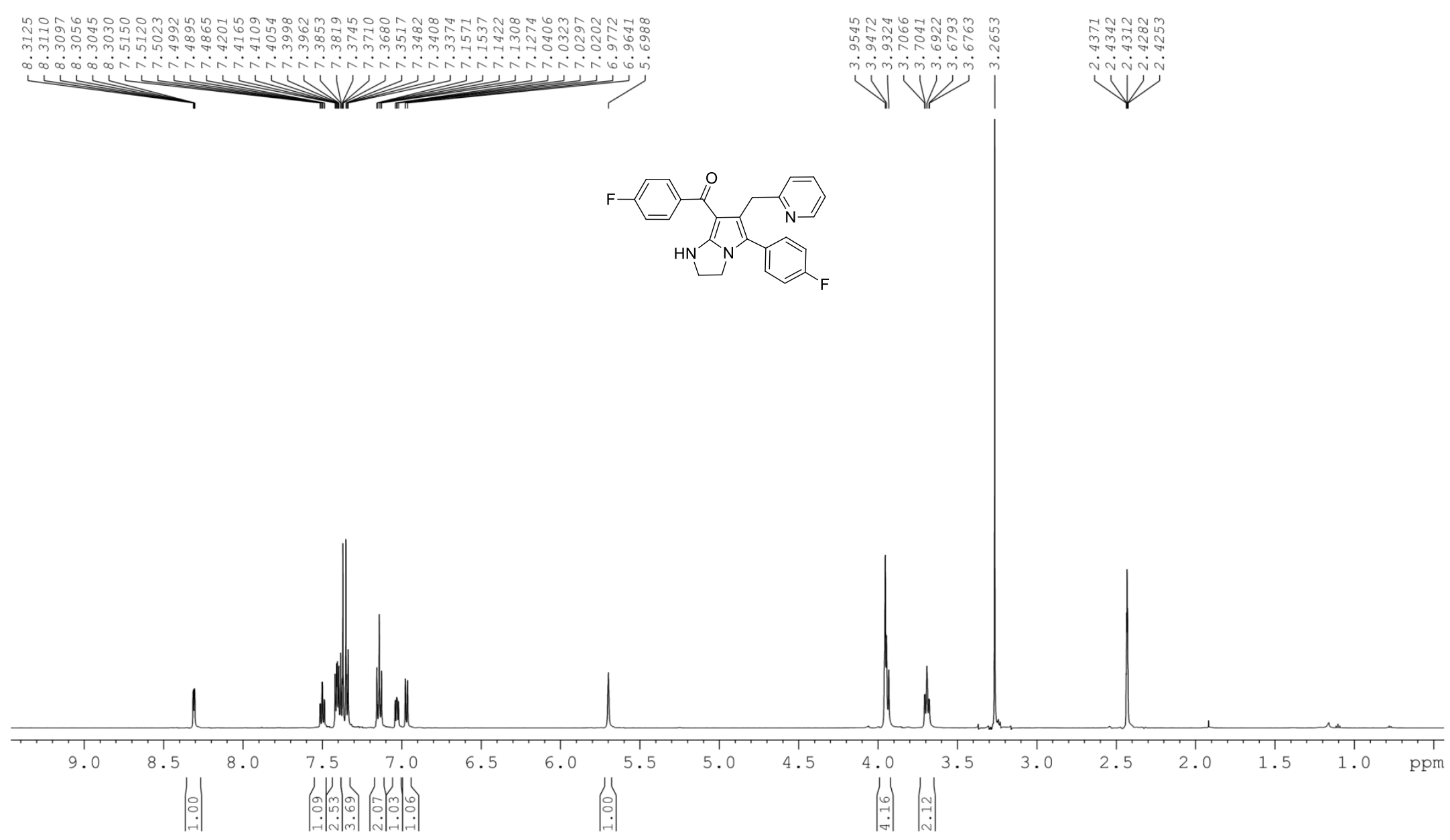

Figure S10. ${ }^{1} \mathrm{H}$ NMR (600 MHz, DMSO- $d_{6}$ ) spectra of compound 4a 


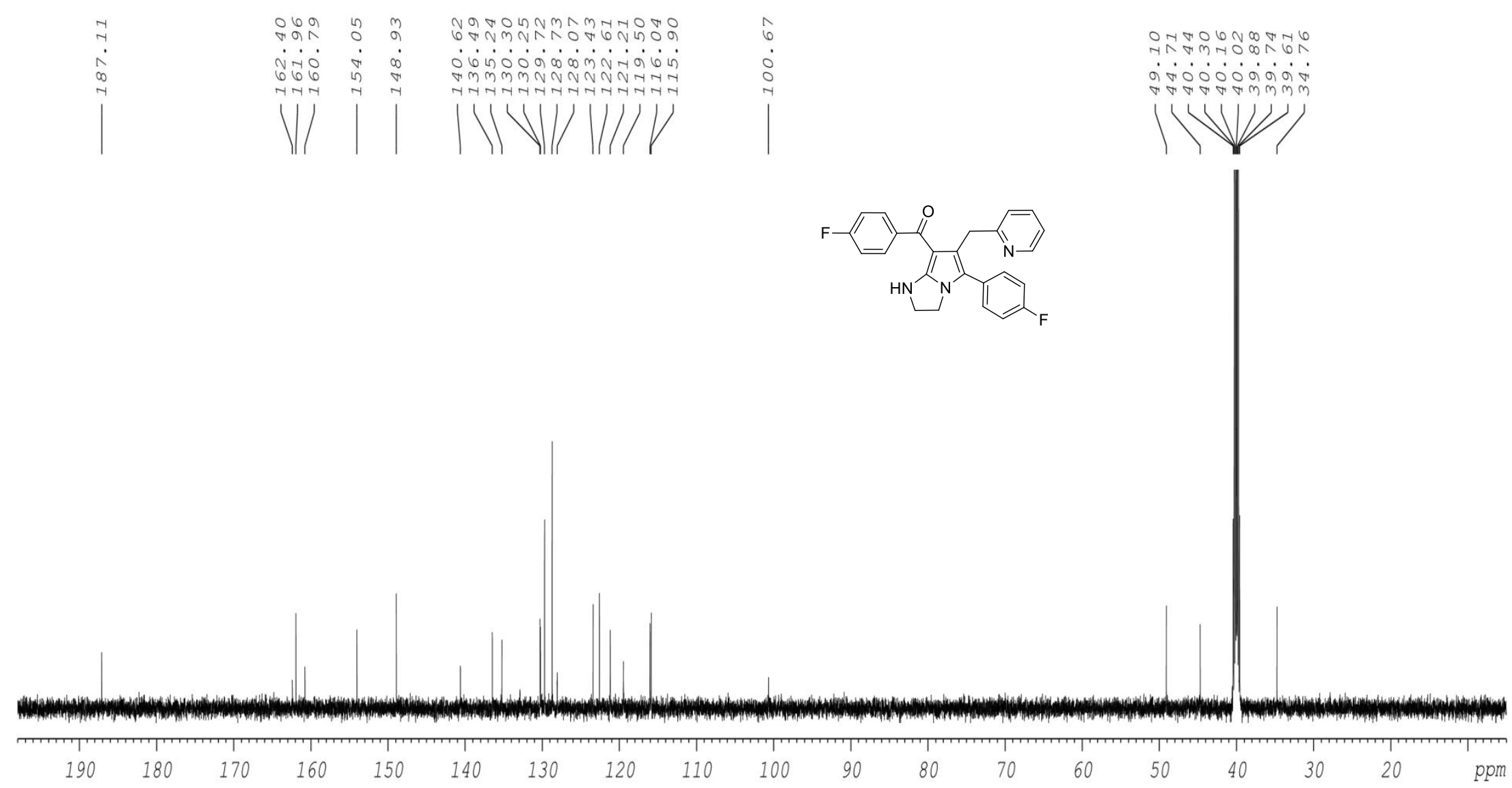

Figure S11. ${ }^{13} \mathrm{C}\left\{{ }^{1} \mathrm{H}\right\}$ NMR $\left(150 \mathrm{MHz}, \mathrm{DMSO}-d_{6}\right)$ spectra of compound 4a 


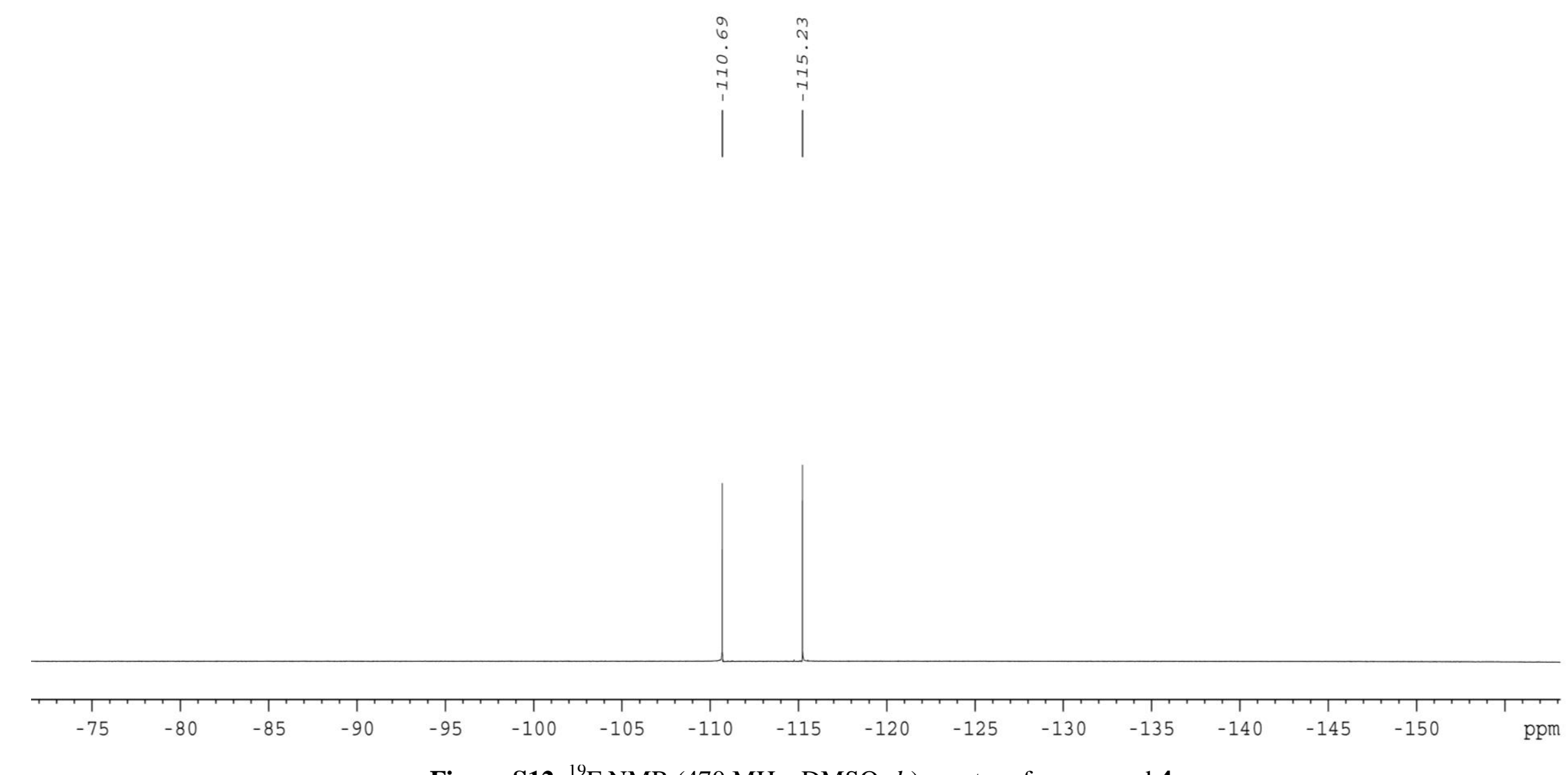

Figure S12. ${ }^{19}$ F NMR $\left(470 \mathrm{MHz}, \mathrm{DMSO}-d_{6}\right)$ spectra of compound $4 \mathbf{a}$ 

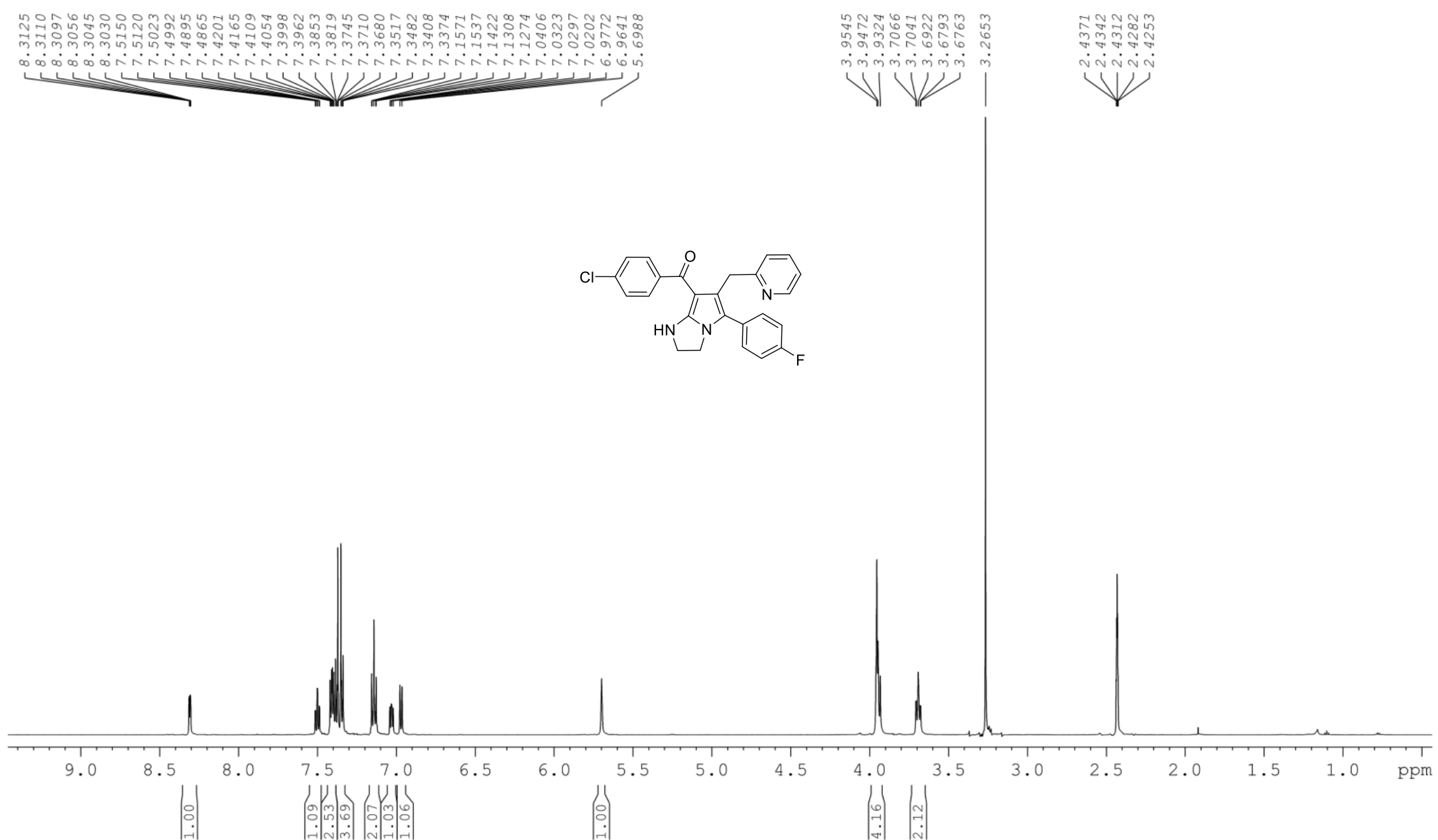

Figure S13. ${ }^{1} \mathrm{H}$ NMR (600 MHz, DMSO- $\left.d_{6}\right)$ spectra of compound $\mathbf{4 b}$ 


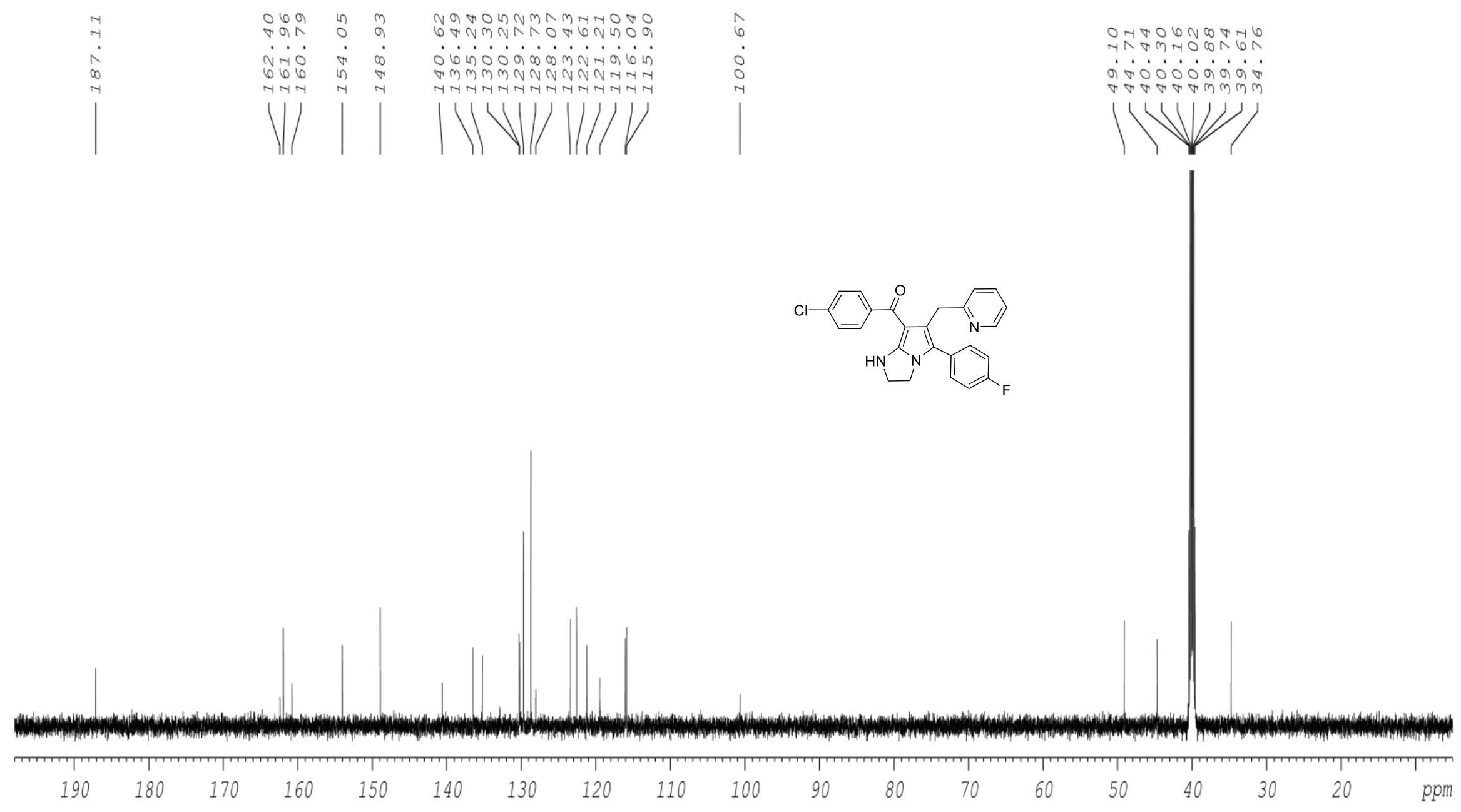

Figure S14. ${ }^{13} \mathrm{C}\left\{{ }^{1} \mathrm{H}\right\}$ NMR (150 MHz, DMSO- $\left.d_{6}\right)$ spectra of compound $\mathbf{4 b}$ 


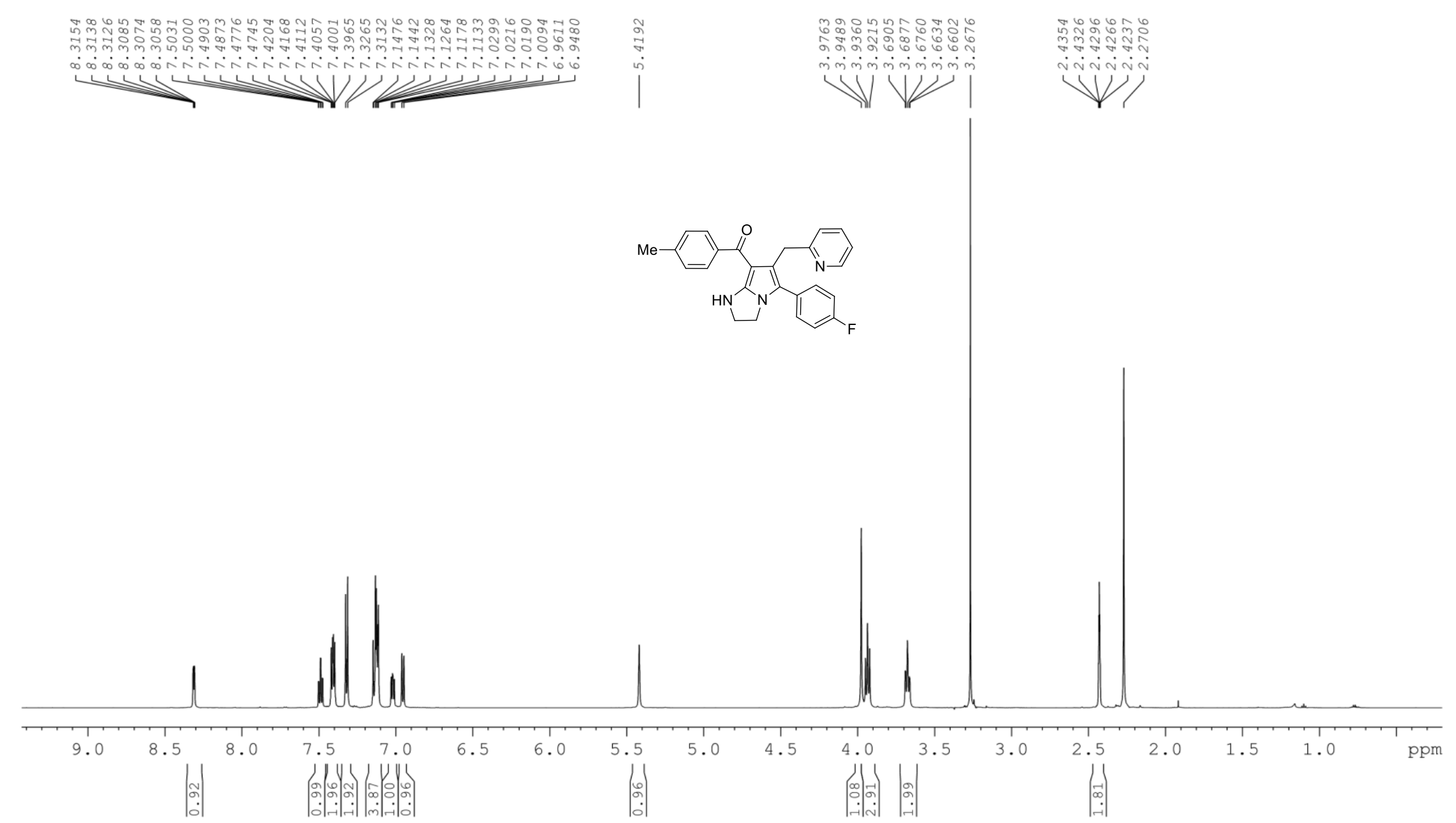

Figure S15. ${ }^{1} \mathrm{H}$ NMR $\left(600 \mathrm{MHz}, \mathrm{DMSO}-d_{6}\right)$ spectra of compound $4 \mathbf{c}$ 


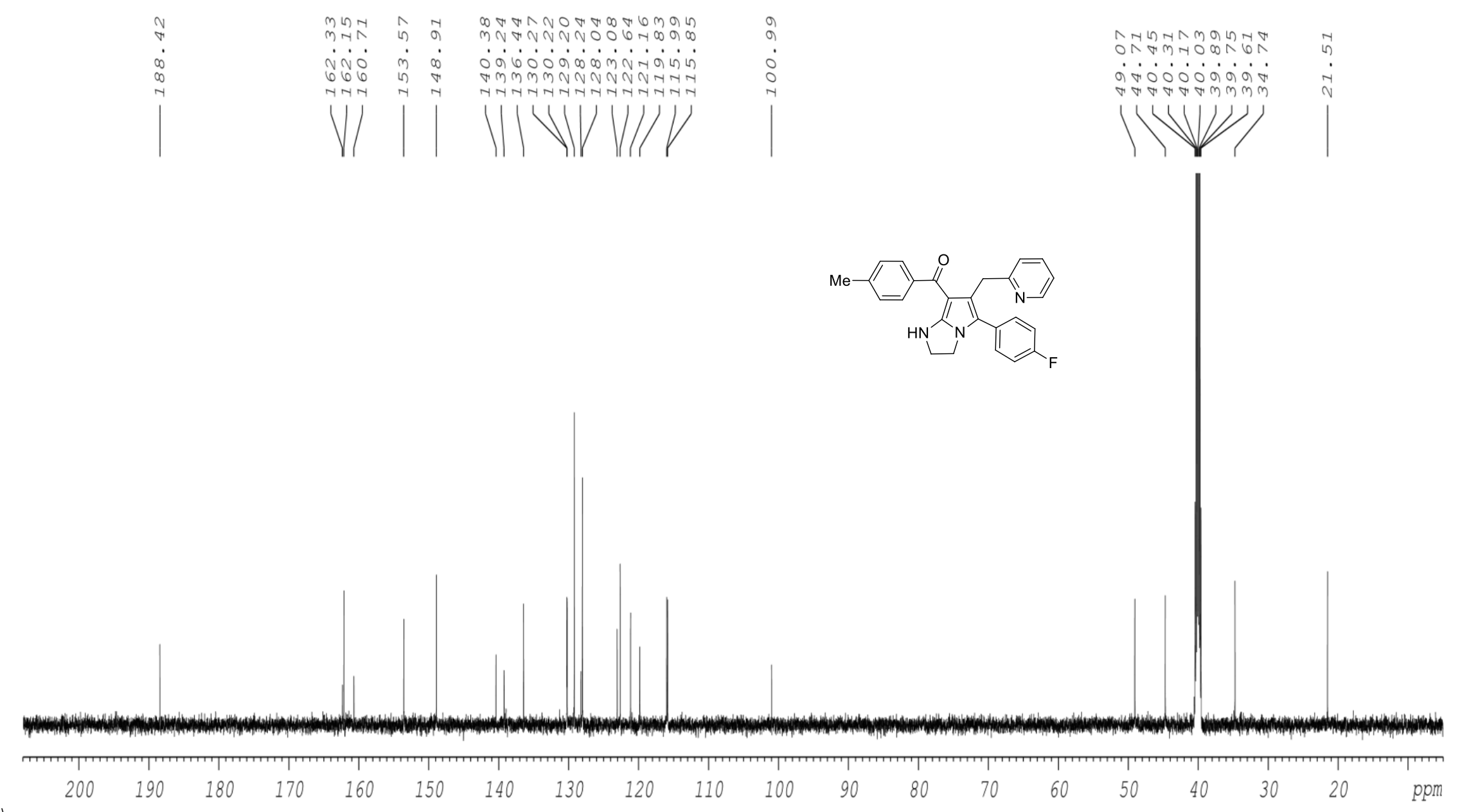

Figure S16. ${ }^{13} \mathrm{C}\left\{{ }^{1} \mathrm{H}\right\}$ NMR $\left(150 \mathrm{MHz}, \mathrm{DMSO}-d_{6}\right)$ spectra of compound $4 \mathbf{c}$ 


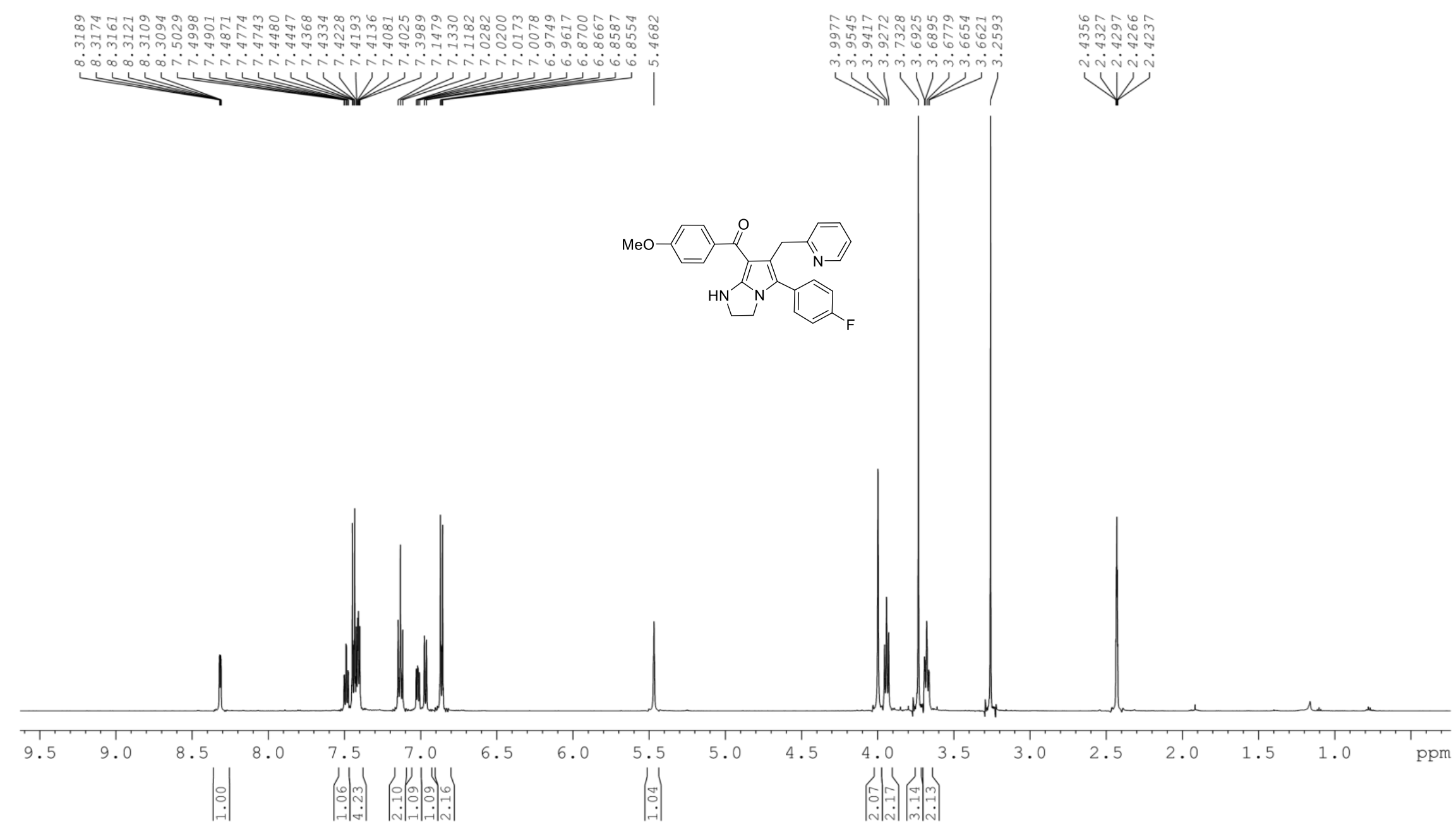

Figure S17. ${ }^{1} \mathrm{H}$ NMR (600 MHz, DMSO- $\left.d_{6}\right)$ spectra of compound $4 \mathbf{d}$ 


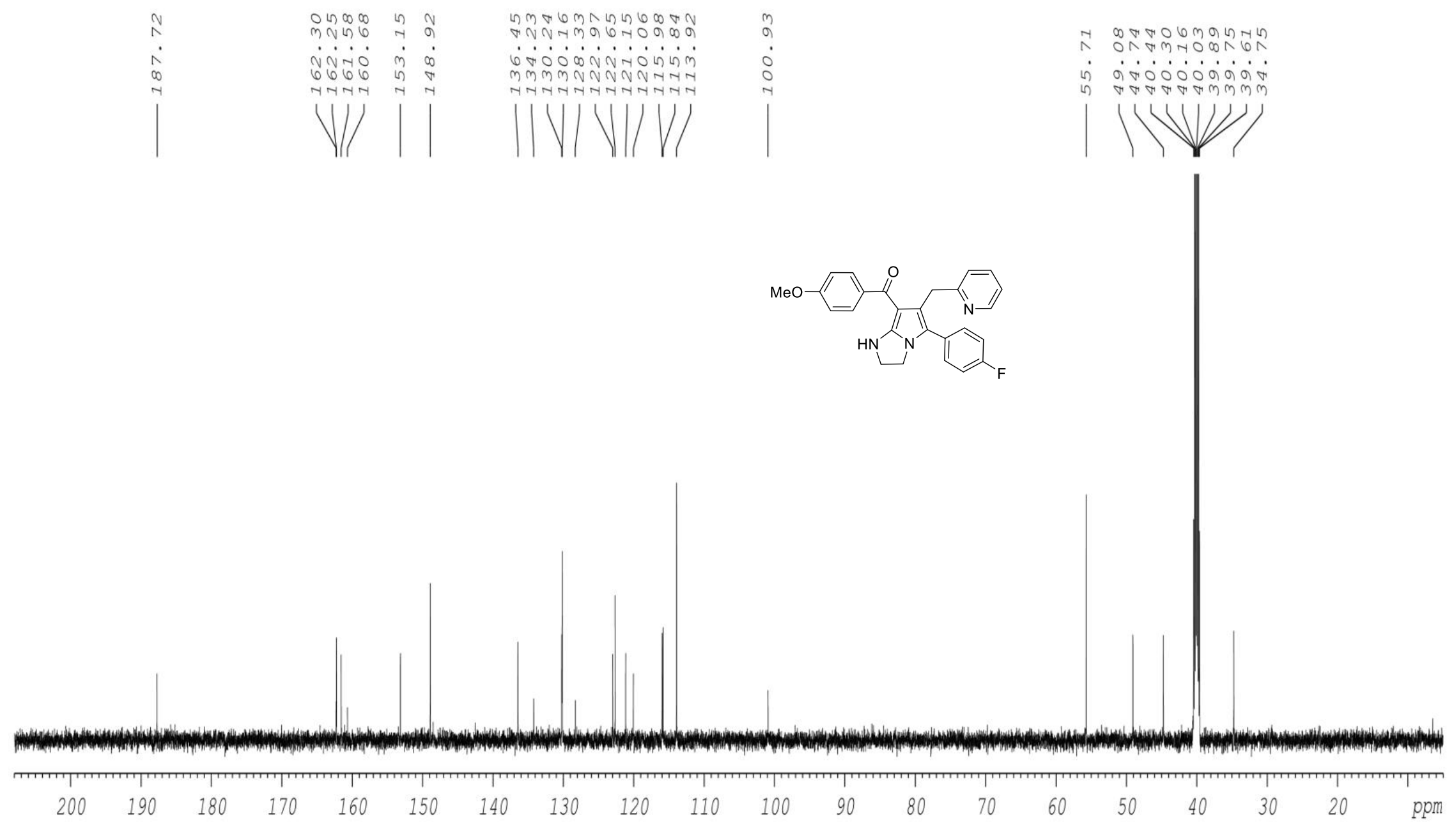

Figure S18. ${ }^{13} \mathrm{C}\left\{{ }^{1} \mathrm{H}\right\}$ NMR $\left(150 \mathrm{MHz}, \mathrm{DMSO}-d_{6}\right)$ spectra of compound $4 \mathbf{d}$ 


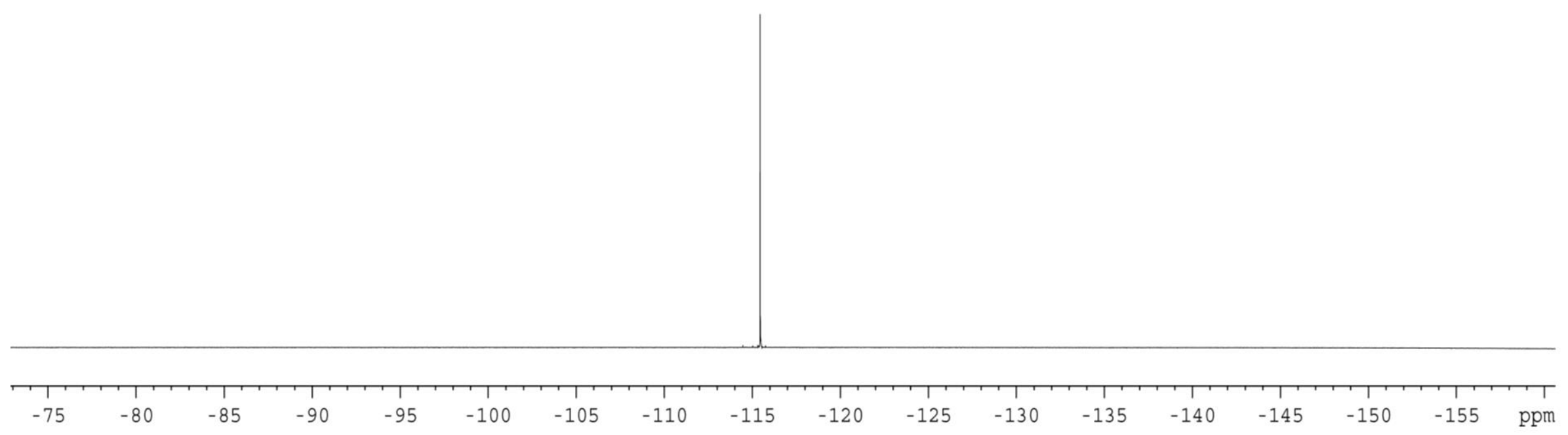

Figure S19. ${ }^{19} \mathrm{~F}$ NMR (470 MHz, DMSO- $\left.d_{6}\right)$ spectra of compound $4 \mathbf{d}$ 


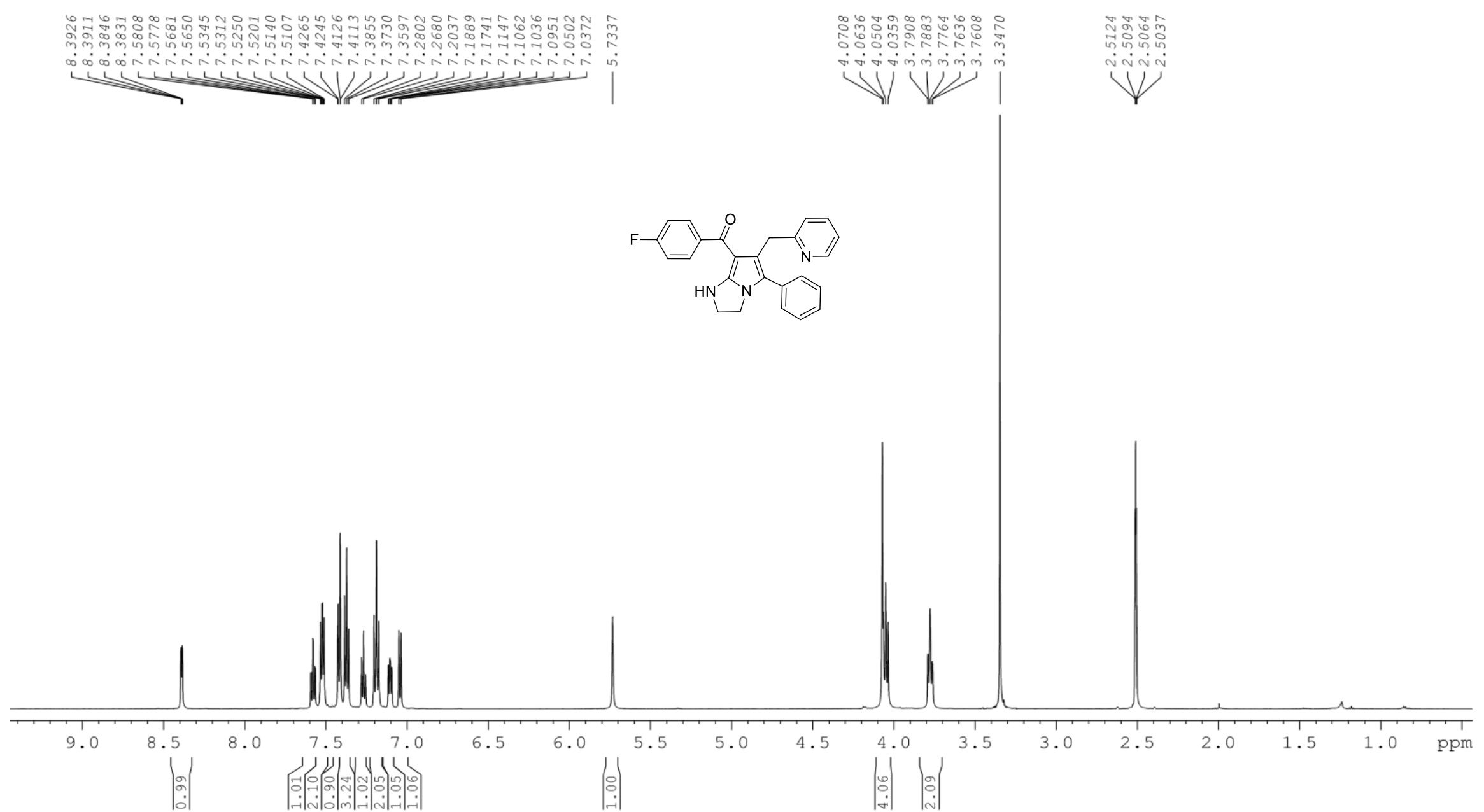

Figure S20. ${ }^{1} \mathrm{H}$ NMR $\left(600 \mathrm{MHz}, \mathrm{DMSO}-d_{6}\right)$ spectra of compound $4 \mathbf{e}$ 


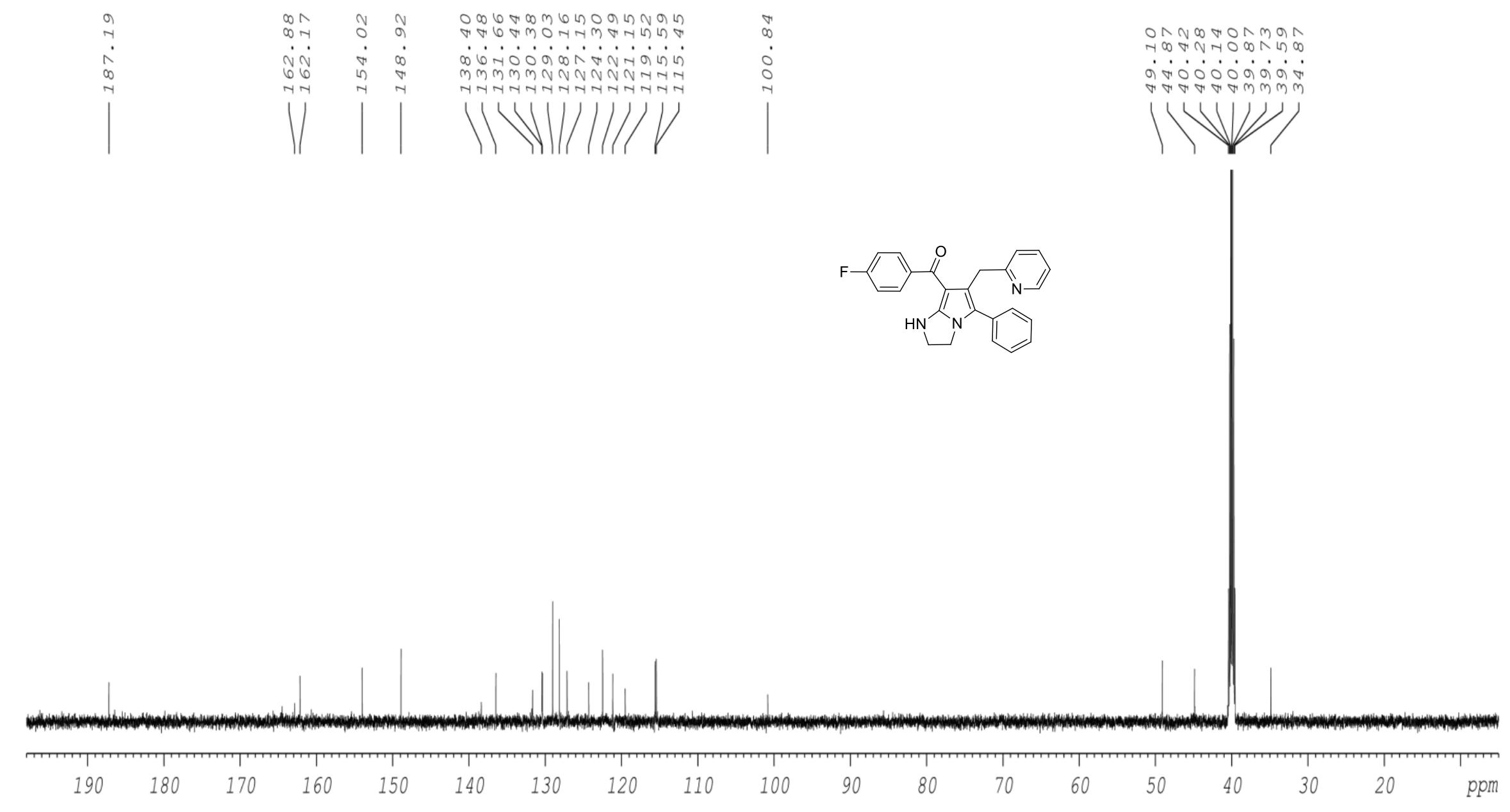

Figure S21. ${ }^{13} \mathrm{C}\left\{{ }^{1} \mathrm{H}\right\}$ NMR (150 MHz, DMSO- $\left.d_{6}\right)$ spectra of compound $4 \mathbf{e}$ 


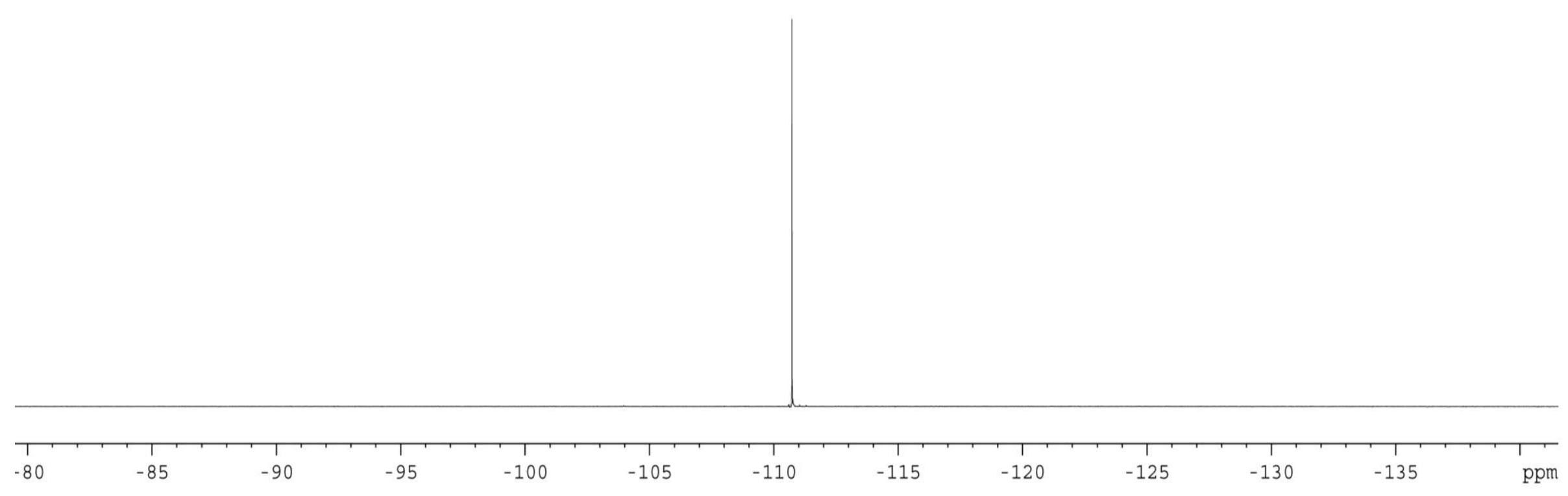

Figure S22. ${ }^{19}$ F NMR (470 MHz, DMSO- $\left.d_{6}\right)$ spectra of compound $4 \mathbf{e}$ 


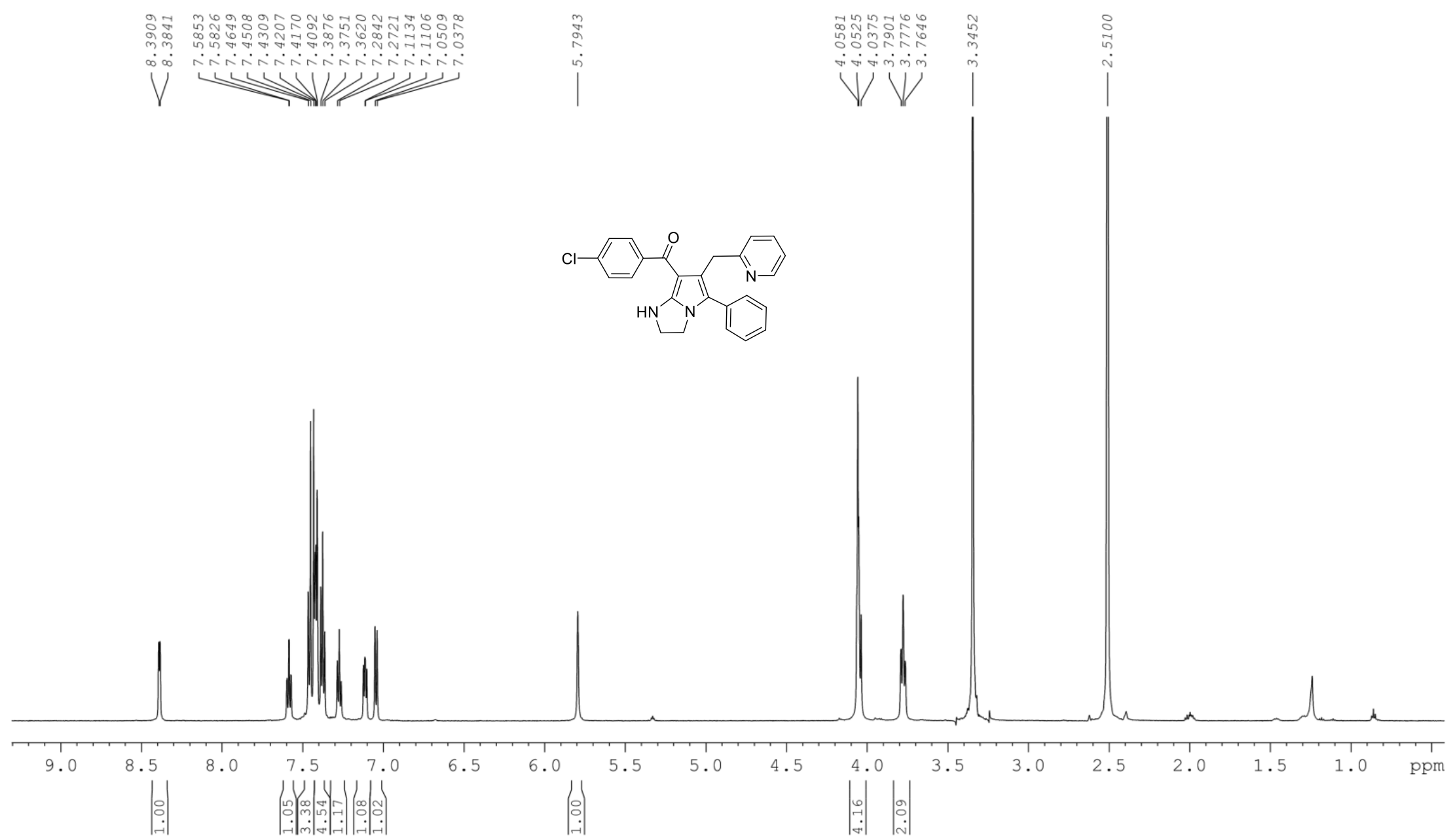

Figure S23. ${ }^{1} \mathrm{H}$ NMR (600 MHz, DMSO- $\left.d_{6}\right)$ spectra of compound $\mathbf{4 f}$ 


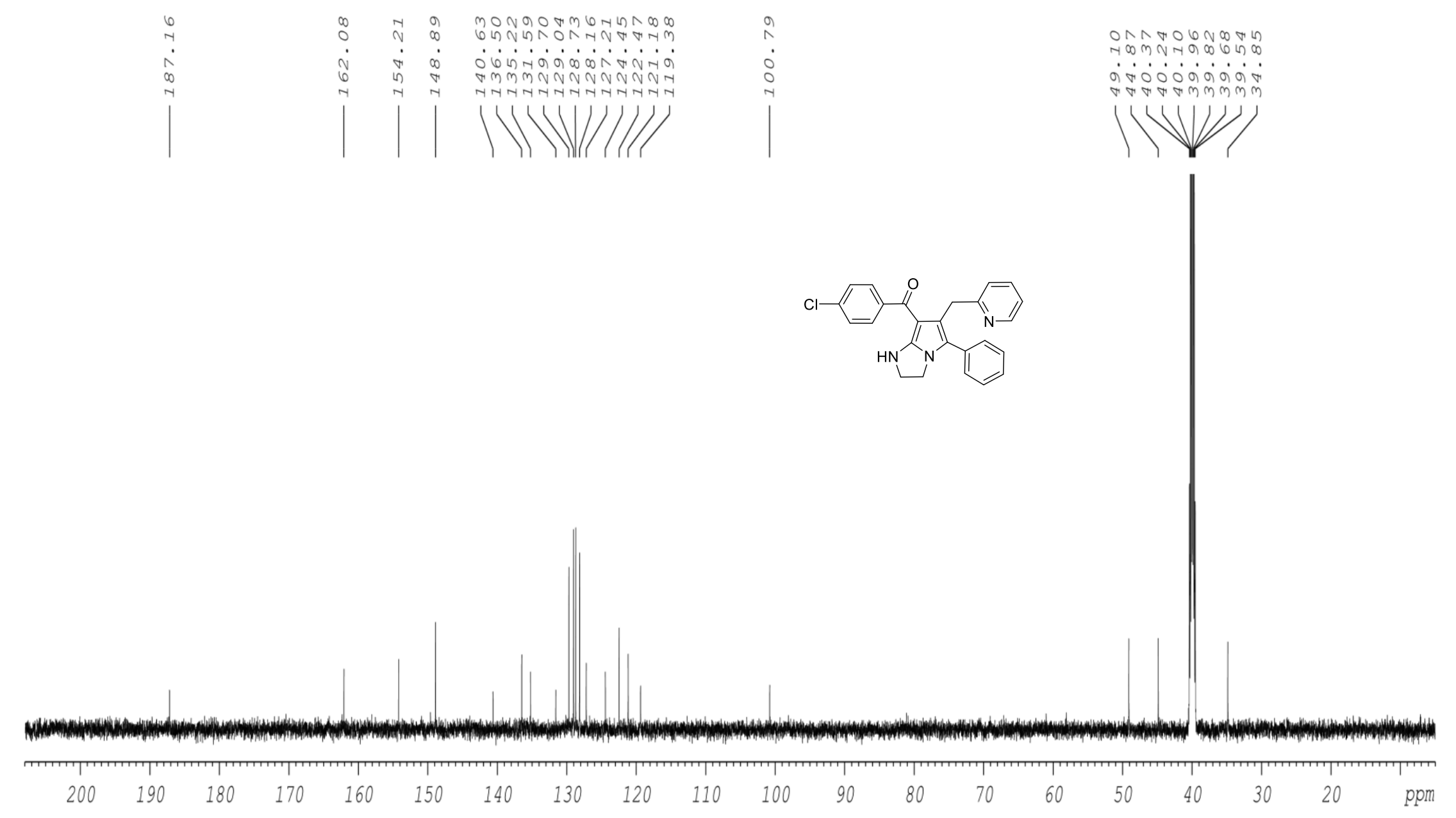

Figure S24. ${ }^{13} \mathrm{C}\left\{{ }^{1} \mathrm{H}\right\} \mathrm{NMR}\left(150 \mathrm{MHz}, \mathrm{DMSO}-d_{6}\right)$ spectra of compound $\mathbf{4 f}$ 

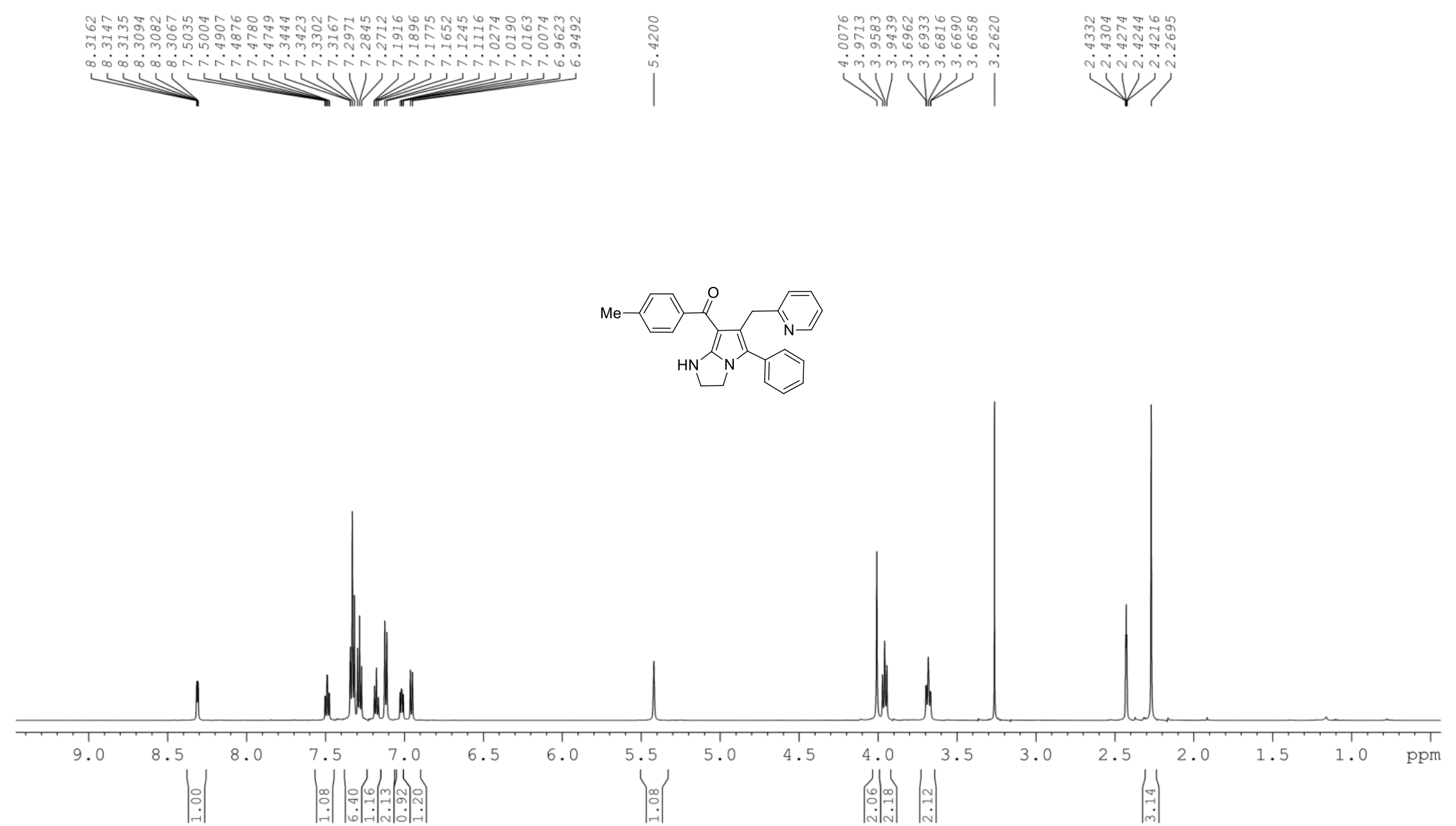

Figure S25. ${ }^{1} \mathrm{H}$ NMR (600 MHz, DMSO- $\left.d_{6}\right)$ spectra of compound $\mathbf{4 g}$ 


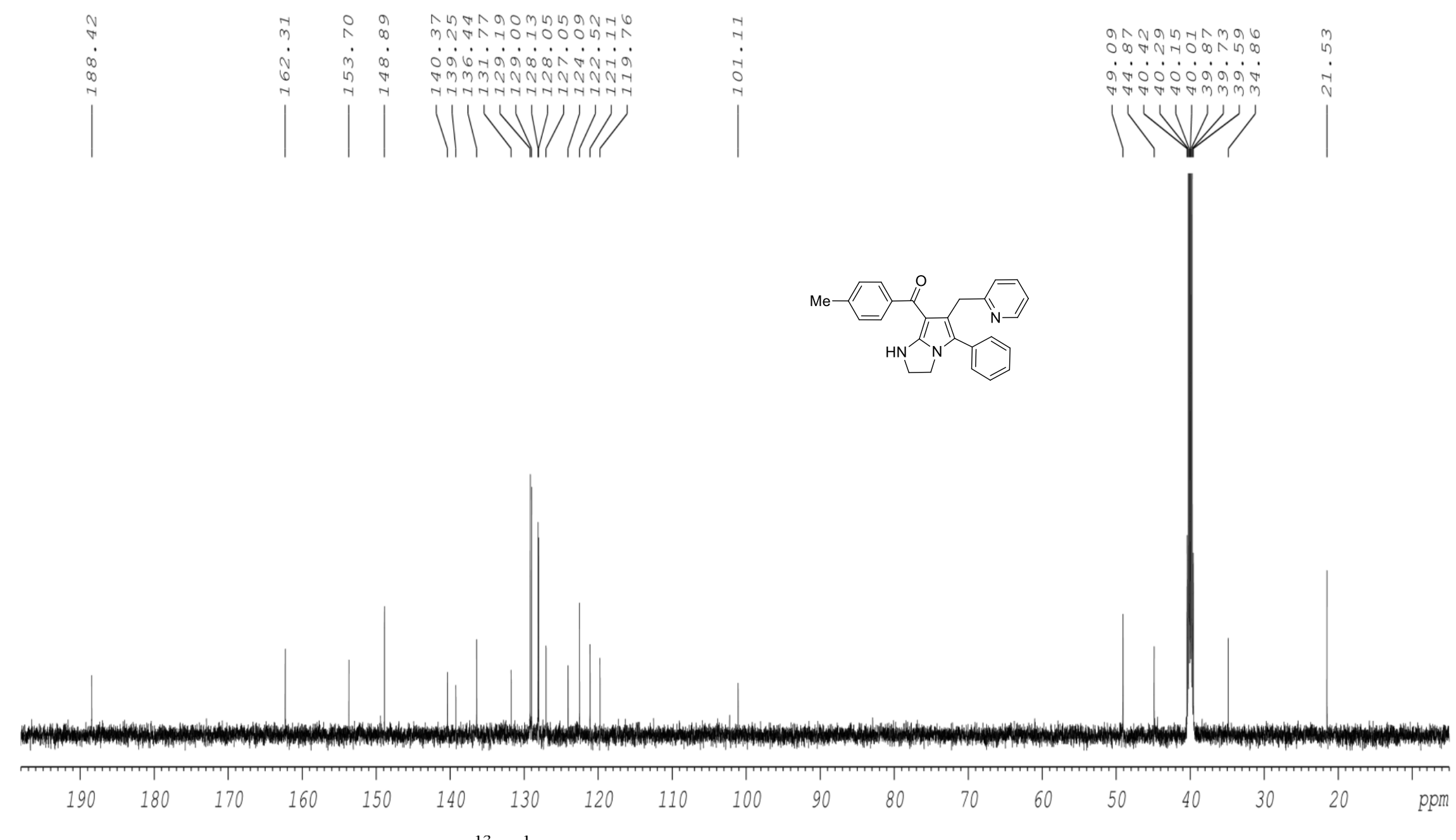

Figure S26. ${ }^{13} \mathrm{C}\left\{{ }^{1} \mathrm{H}\right\}$ NMR $\left(150 \mathrm{MHz}, \mathrm{DMSO}-d_{6}\right)$ spectra of compound $\mathbf{4 g}$ 


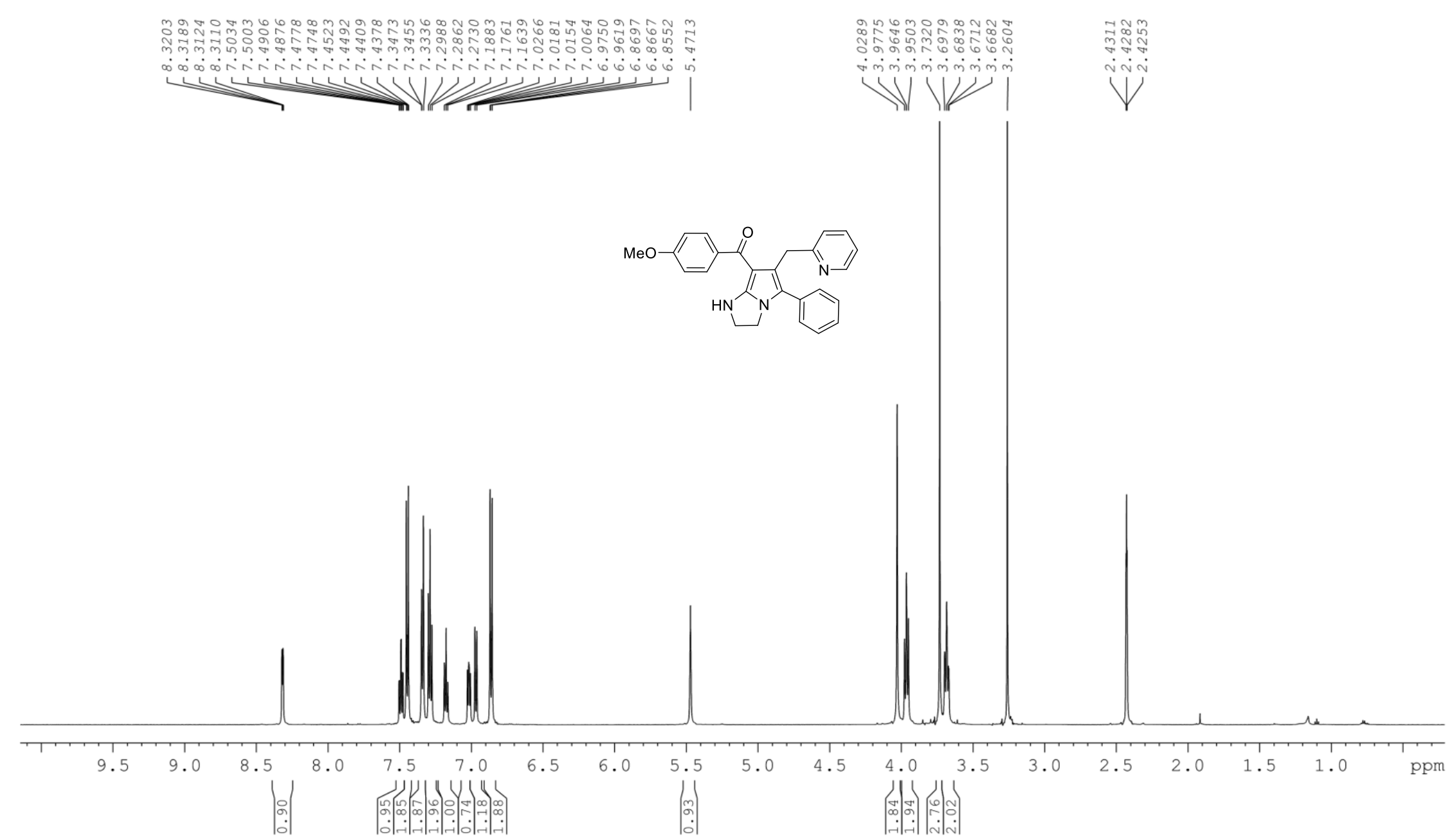

Figure S27. ${ }^{1} \mathrm{H}$ NMR (600 MHz, DMSO- $d_{6}$ ) spectra of compound $\mathbf{4 h}$ 


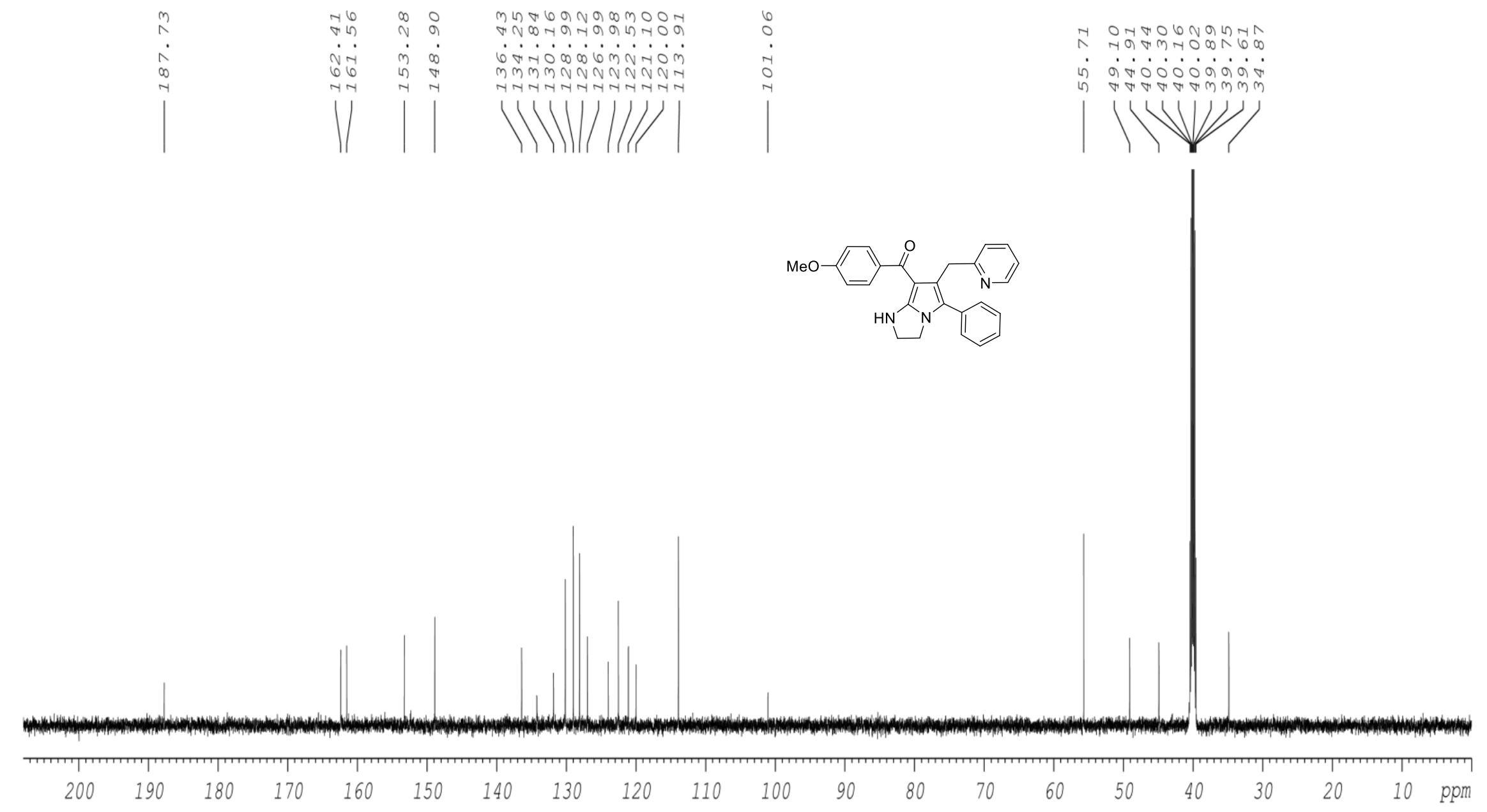

Figure S28. ${ }^{13} \mathrm{C}\left\{{ }^{1} \mathrm{H}\right\}$ NMR (150 MHz, DMSO- $\left.d_{6}\right)$ spectra of compound $\mathbf{4 h}$ 


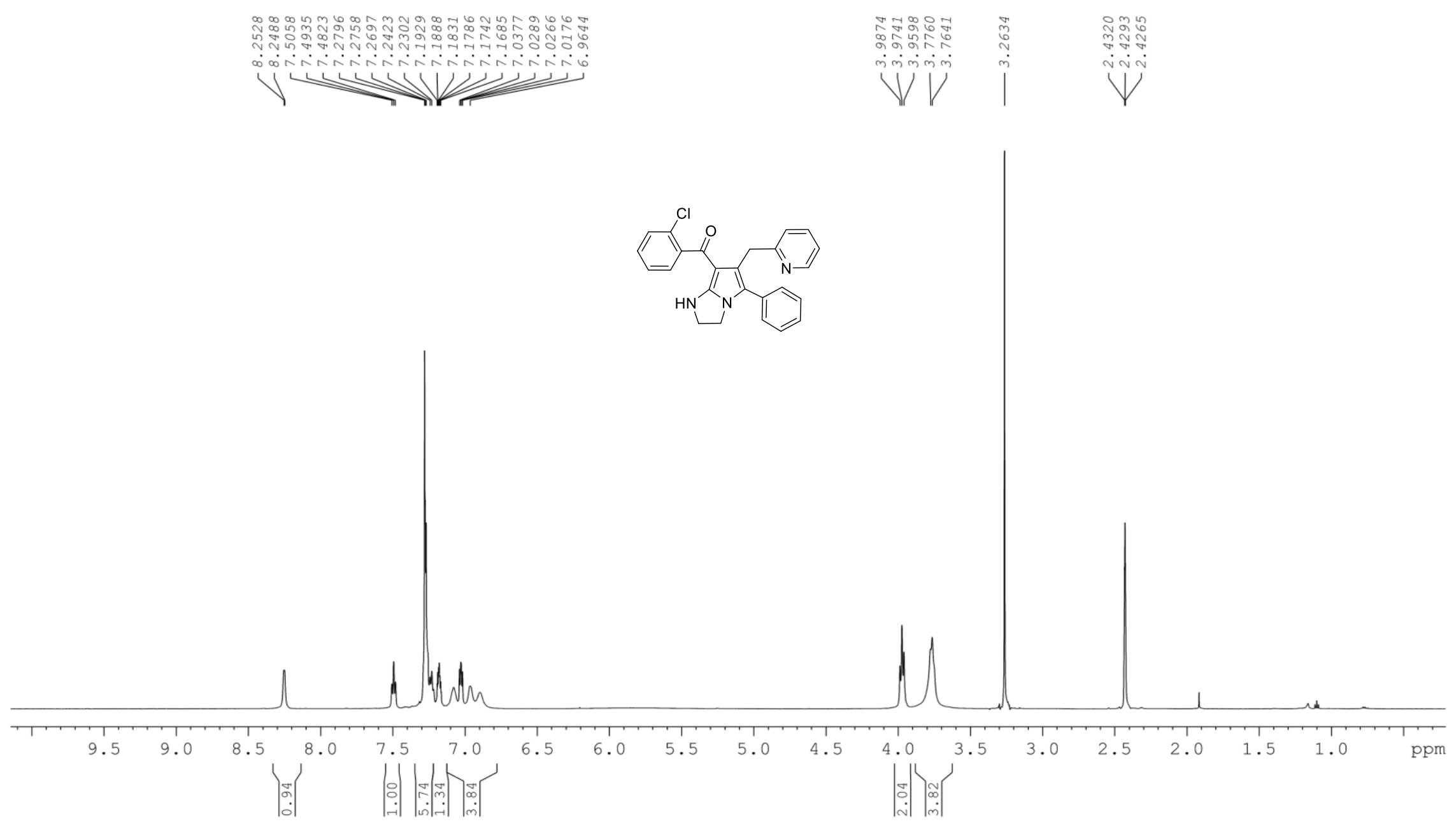

Figure S29. ${ }^{1} \mathrm{H}$ NMR (600 MHz, DMSO- $d_{6}$ ) spectra of compound $4 \mathbf{i}$ 


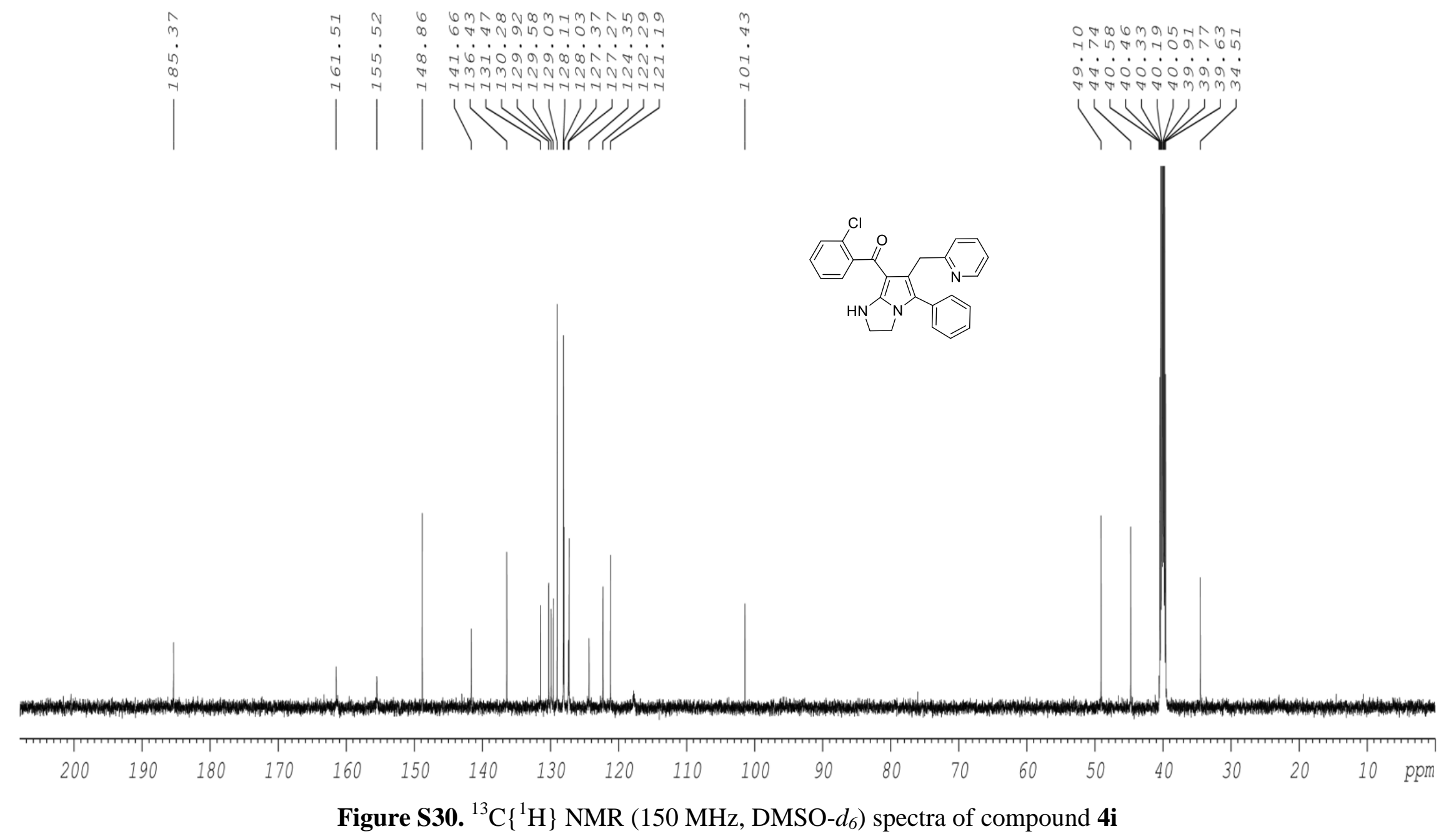



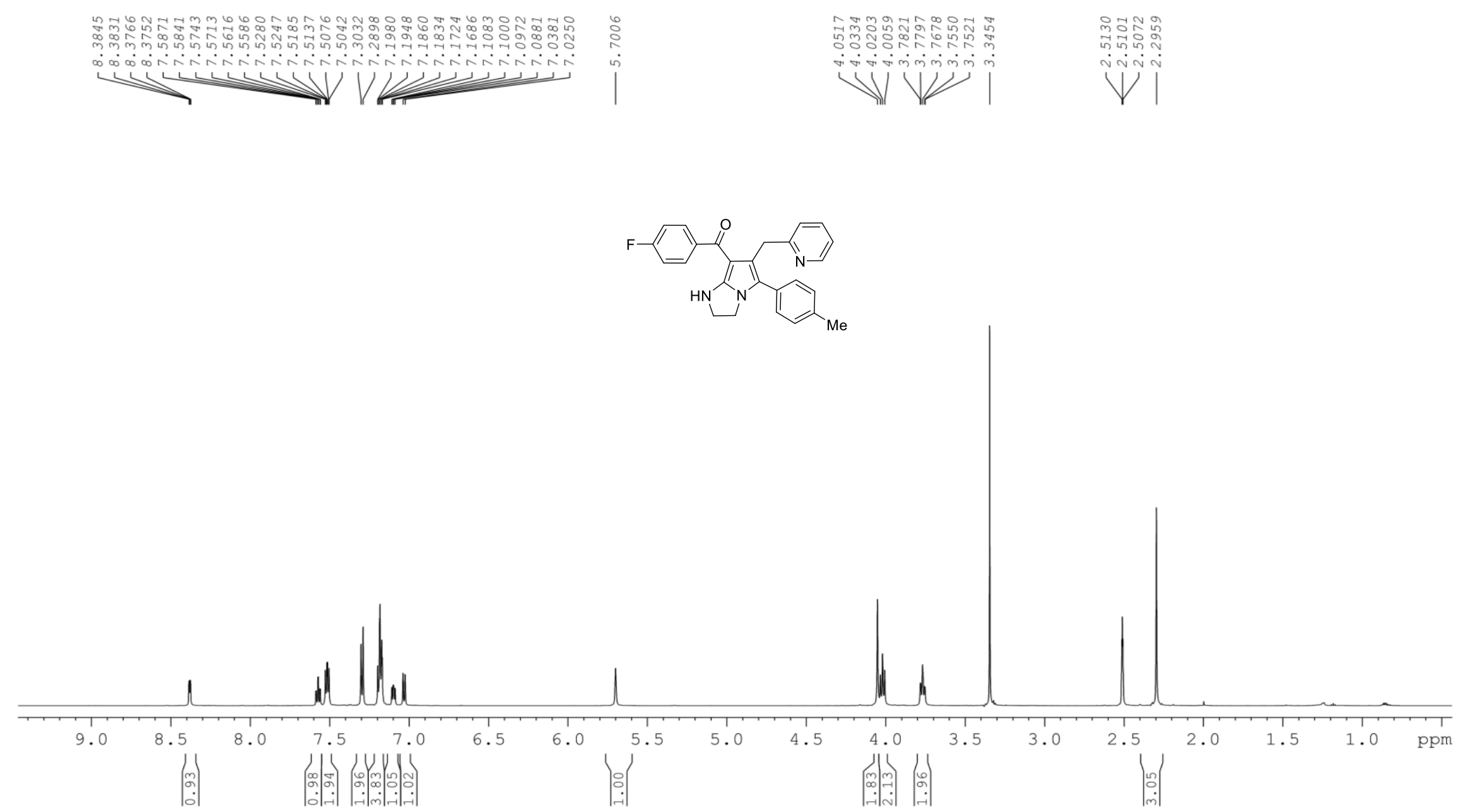

Figure S31. ${ }^{1} \mathrm{H}$ NMR (600 MHz, DMSO- $\left.d_{6}\right)$ spectra of compound $\mathbf{4 j}$ 


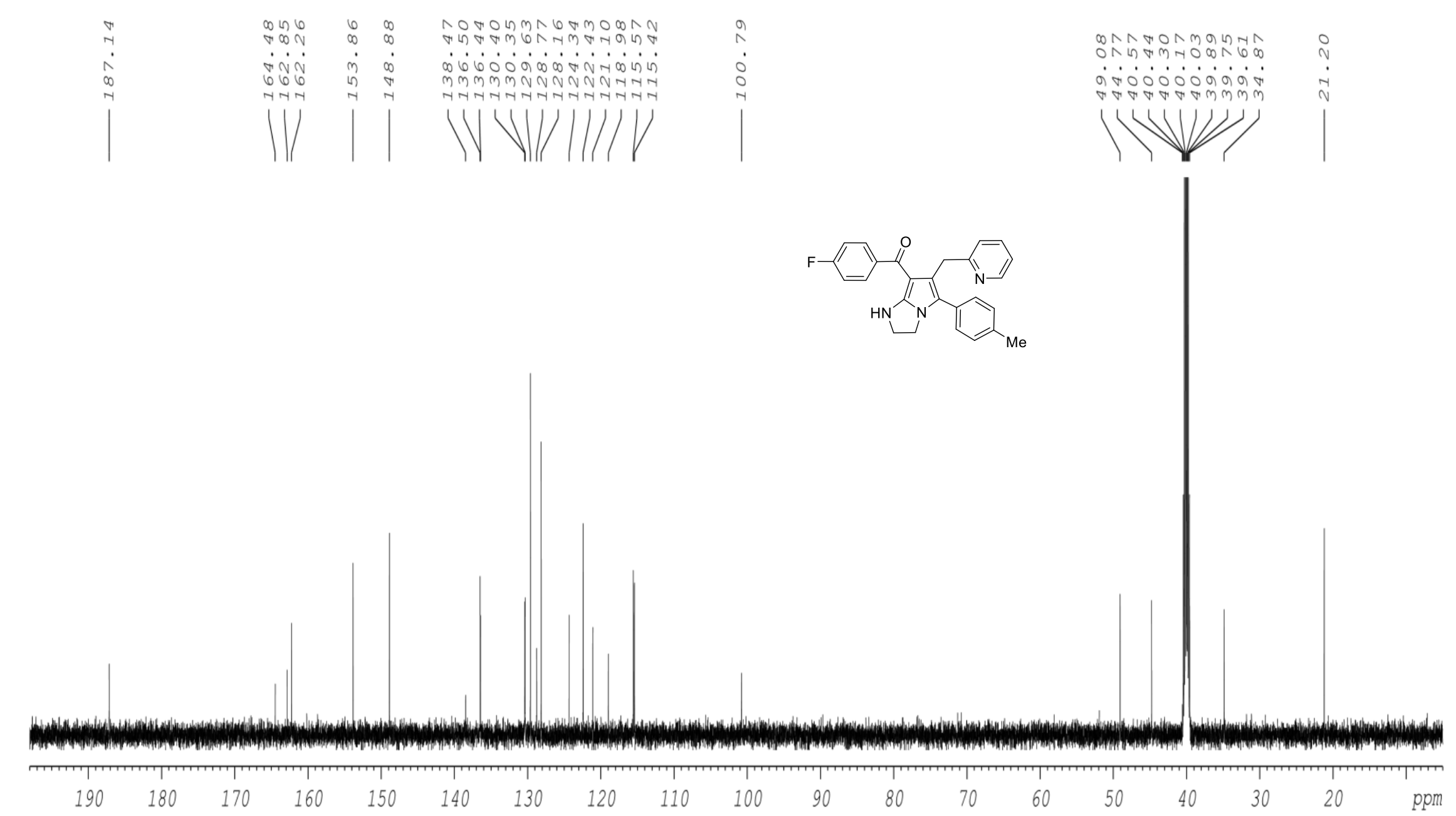

Figure S32. ${ }^{13} \mathrm{C}\left\{{ }^{1} \mathrm{H}\right\}$ NMR $\left(150 \mathrm{MHz}, \mathrm{DMSO}-d_{6}\right)$ spectra of compound $\mathbf{4 j}$ 


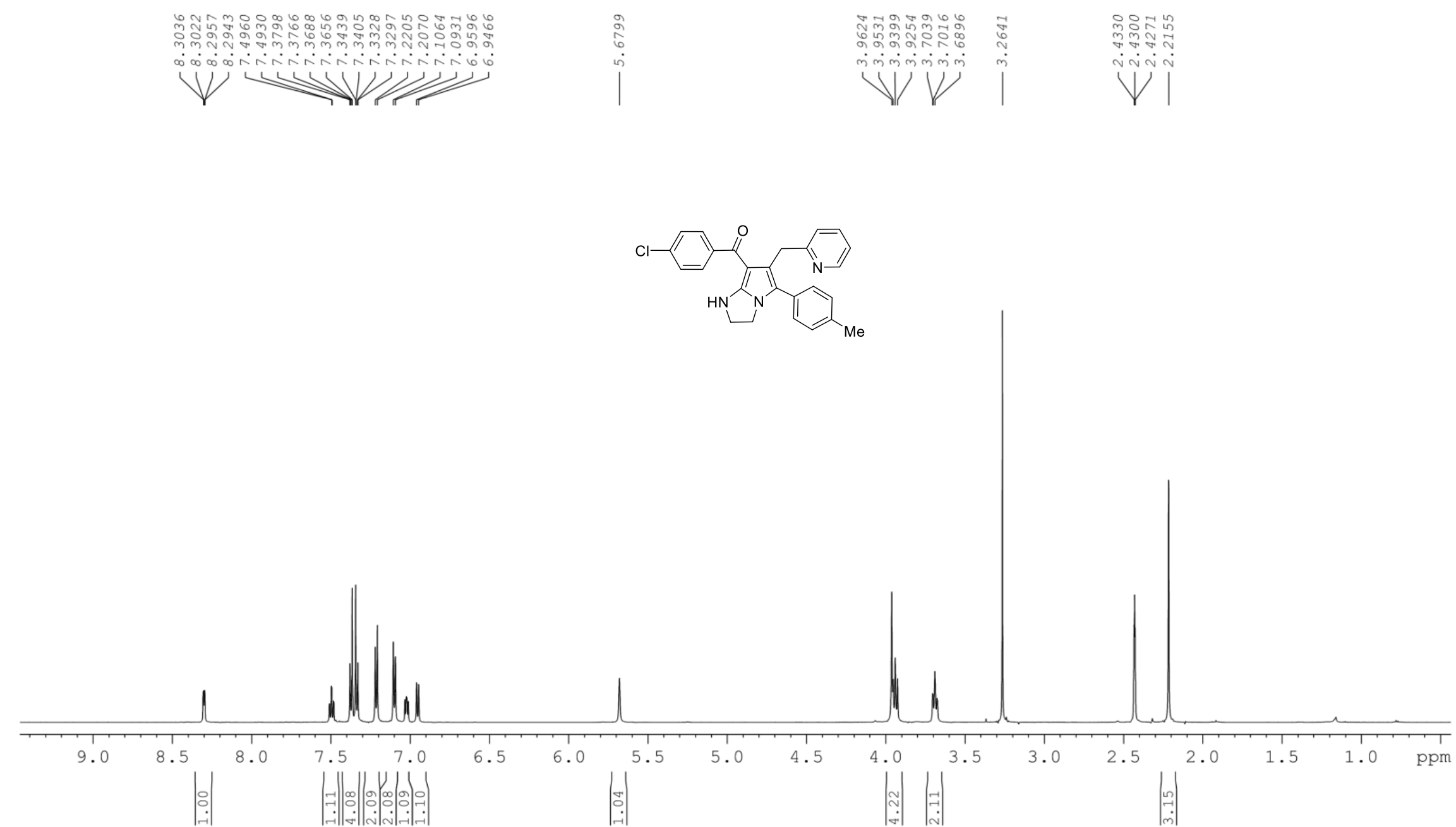

Figure S33. ${ }^{1} \mathrm{H}$ NMR (600 MHz, DMSO- $\left.d_{6}\right)$ spectra of compound $4 \mathbf{k}$ 


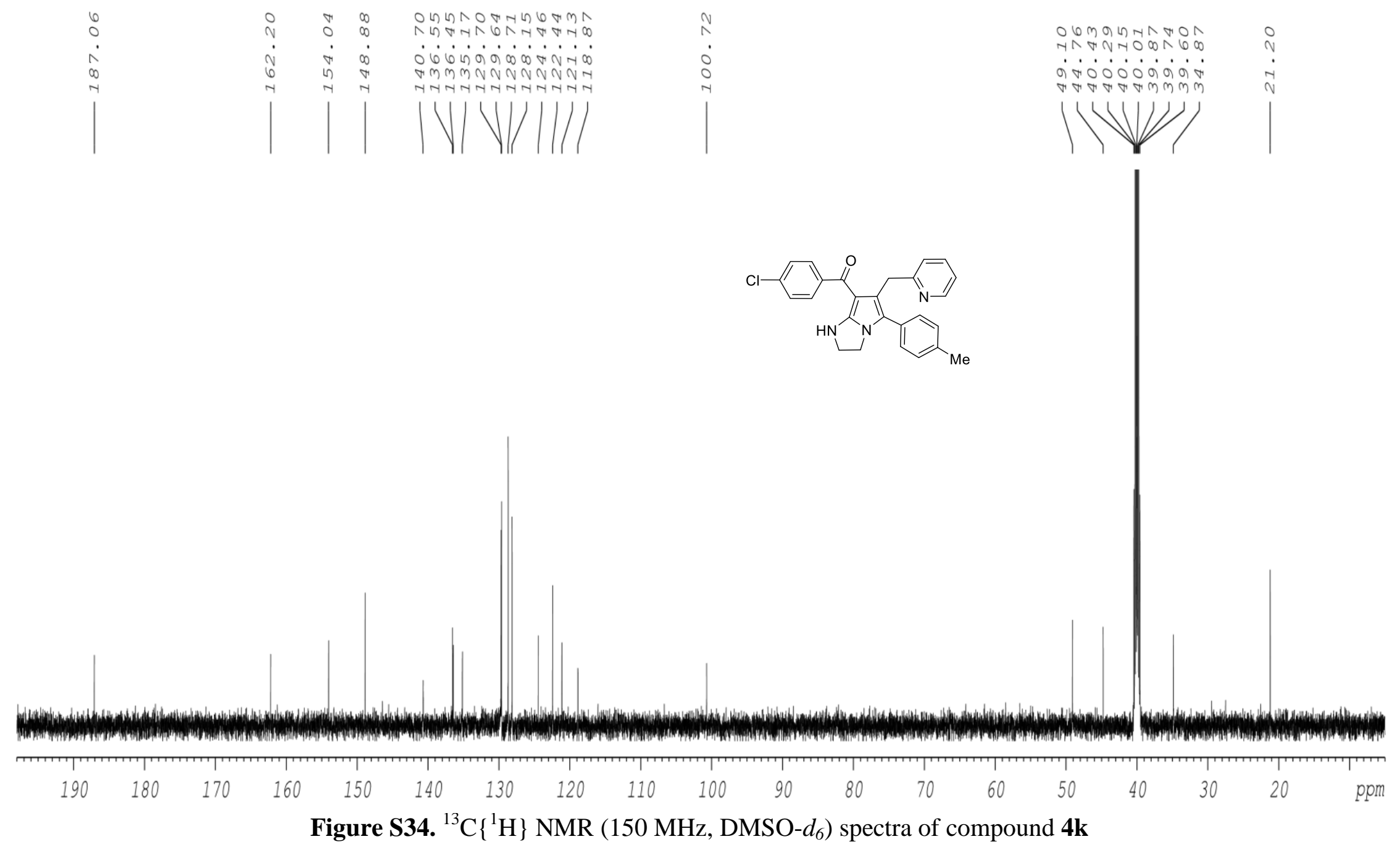




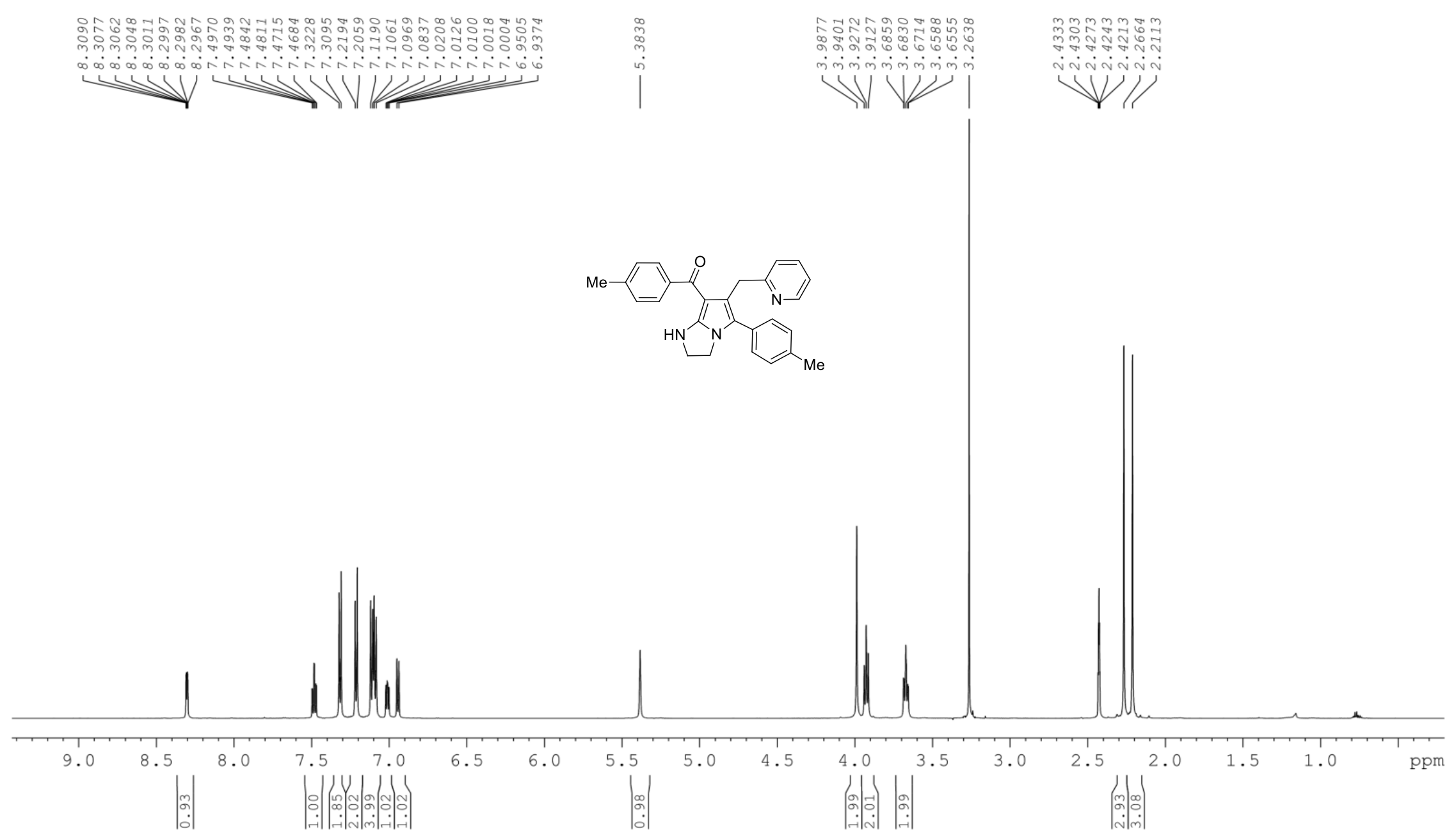

Figure S35. ${ }^{1} \mathrm{H}$ NMR (600 MHz, DMSO- $\left.d_{6}\right)$ spectra of compound 41 


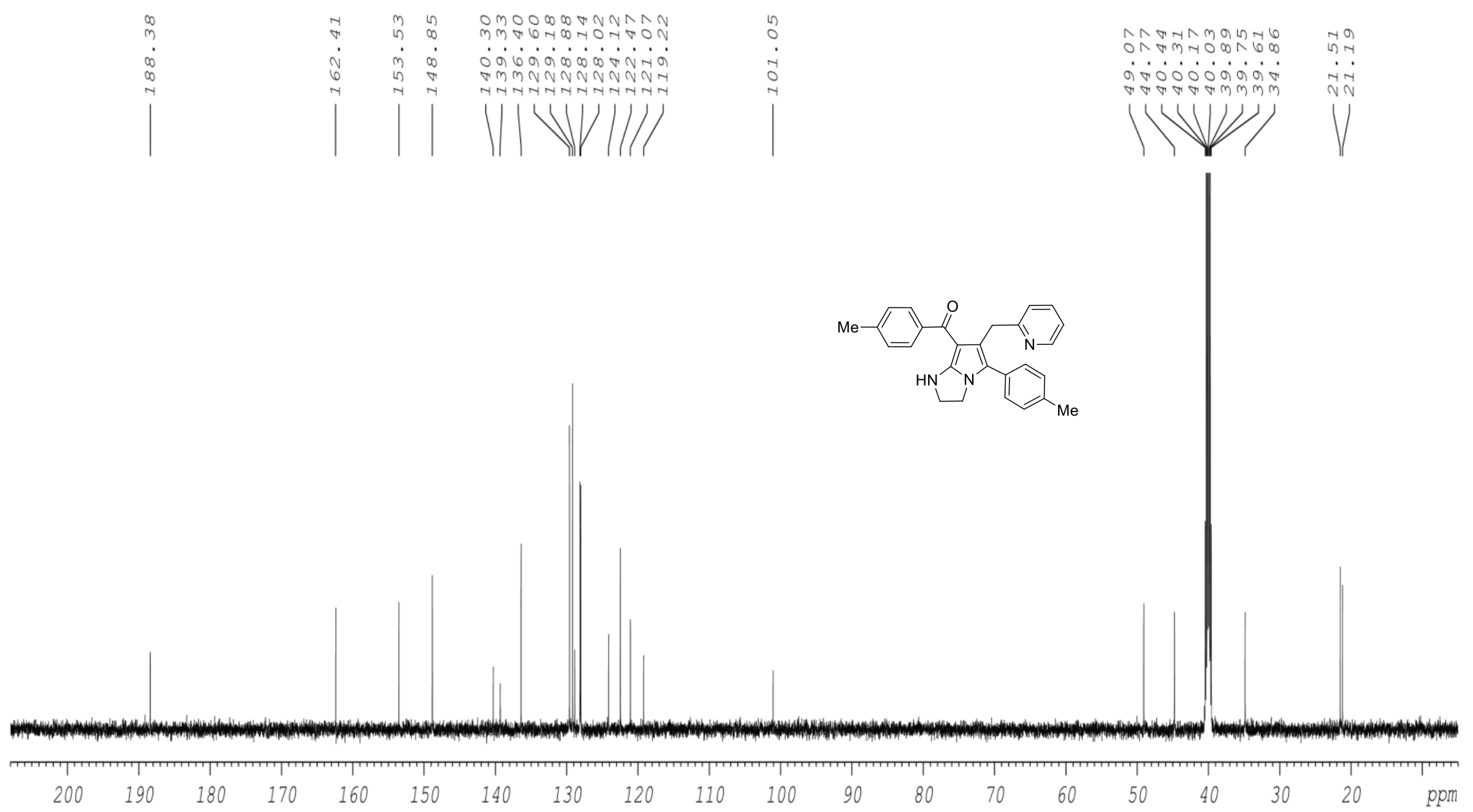

Figure S36. ${ }^{13} \mathrm{C}\left\{{ }^{1} \mathrm{H}\right\}$ NMR $\left(150 \mathrm{MHz}, \mathrm{DMSO}-d_{6}\right)$ spectra of compound $4 \mathbf{l}$ 


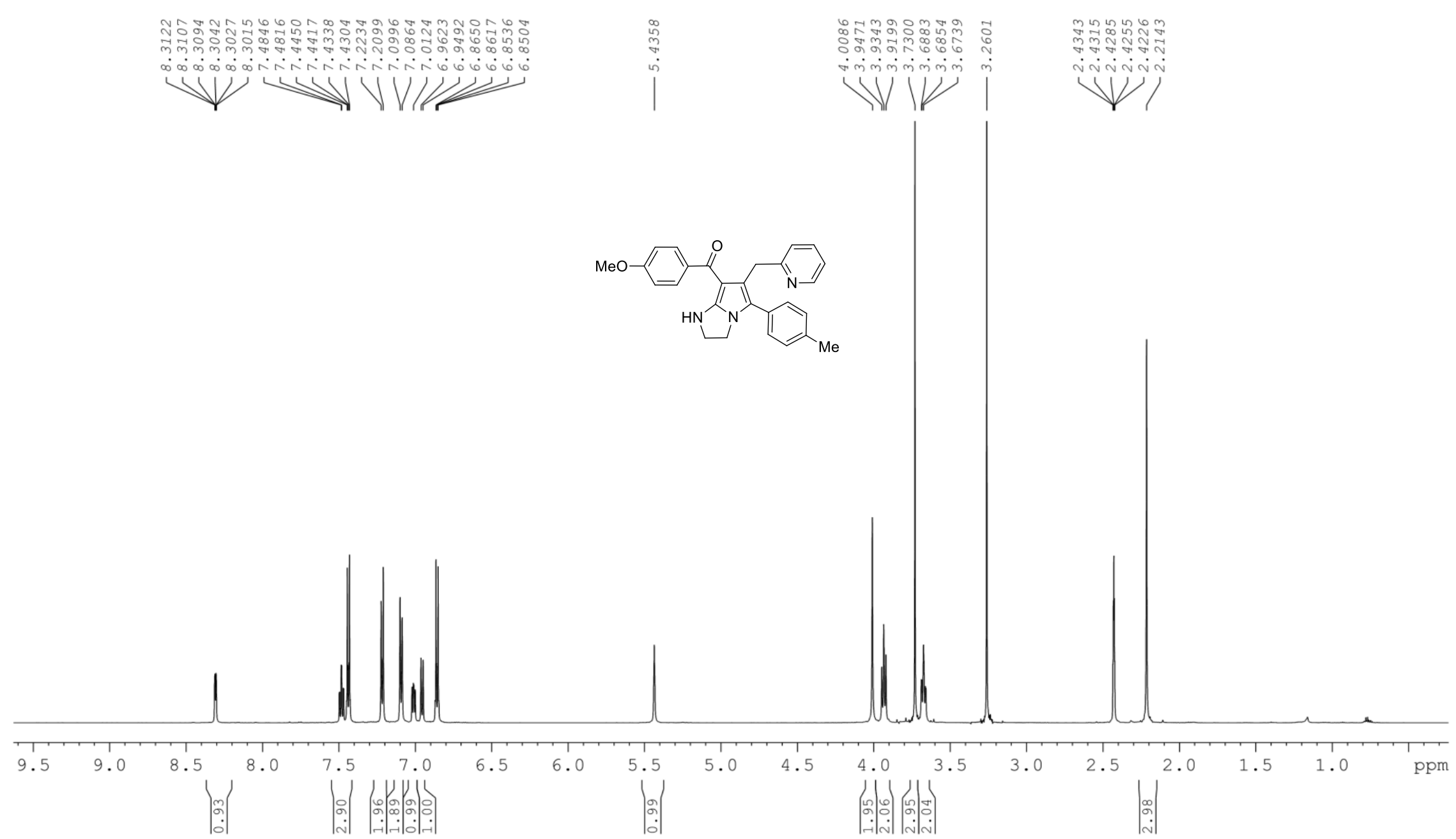

Figure S37. ${ }^{1} \mathrm{H}$ NMR $\left(600 \mathrm{MHz}, \mathrm{DMSO}-d_{6}\right)$ spectra of compound $\mathbf{4 m}$ 


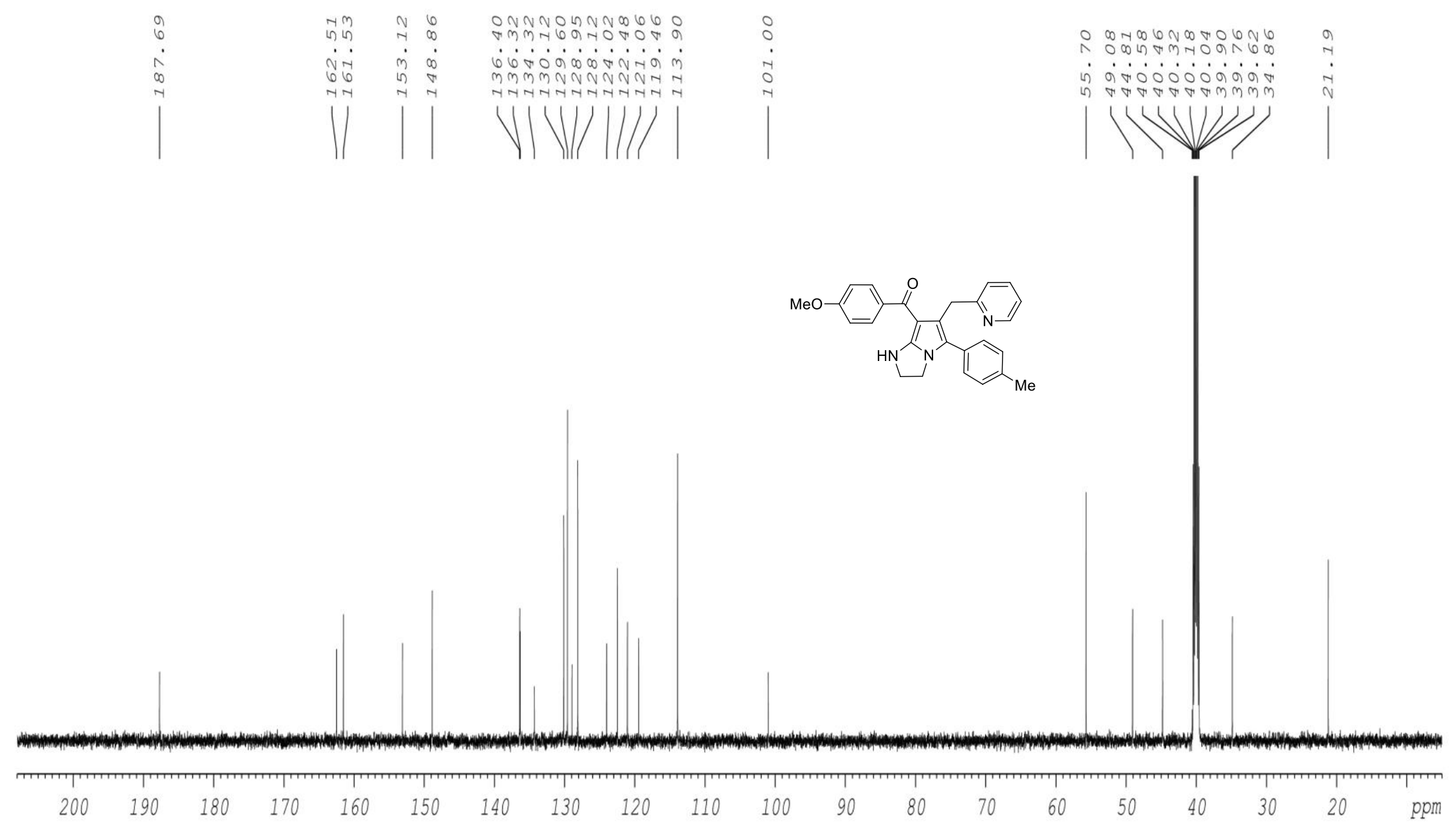

Figure S38. ${ }^{13} \mathrm{C}\left\{{ }^{1} \mathrm{H}\right\}$ NMR (150 MHz, DMSO- $\left.d_{6}\right)$ spectra of compound $\mathbf{4 m}$ 


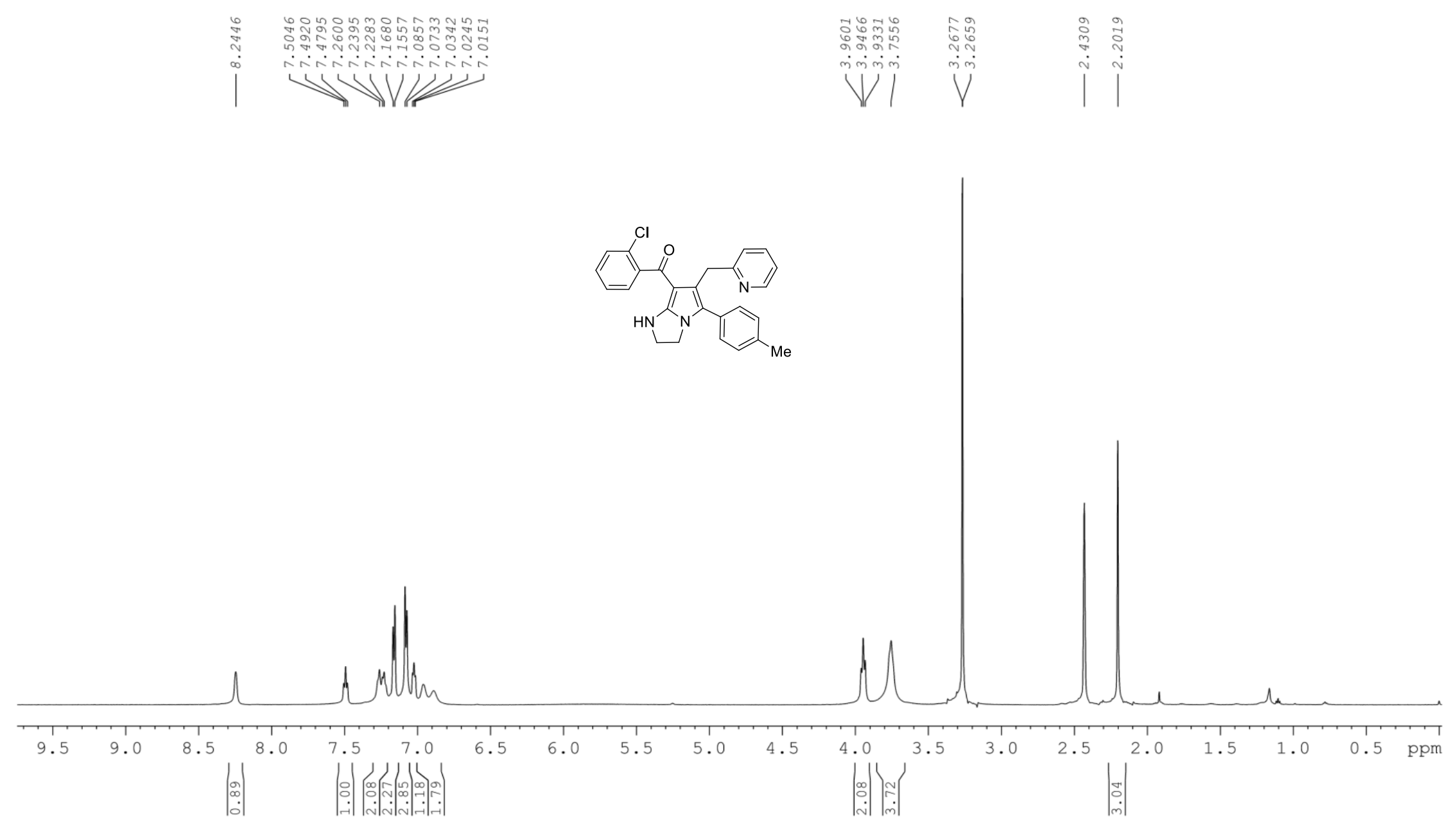

Figure S39. ${ }^{1} \mathrm{H}$ NMR (600 MHz, DMSO- $\left.d_{6}\right)$ spectra of compound 4 n 


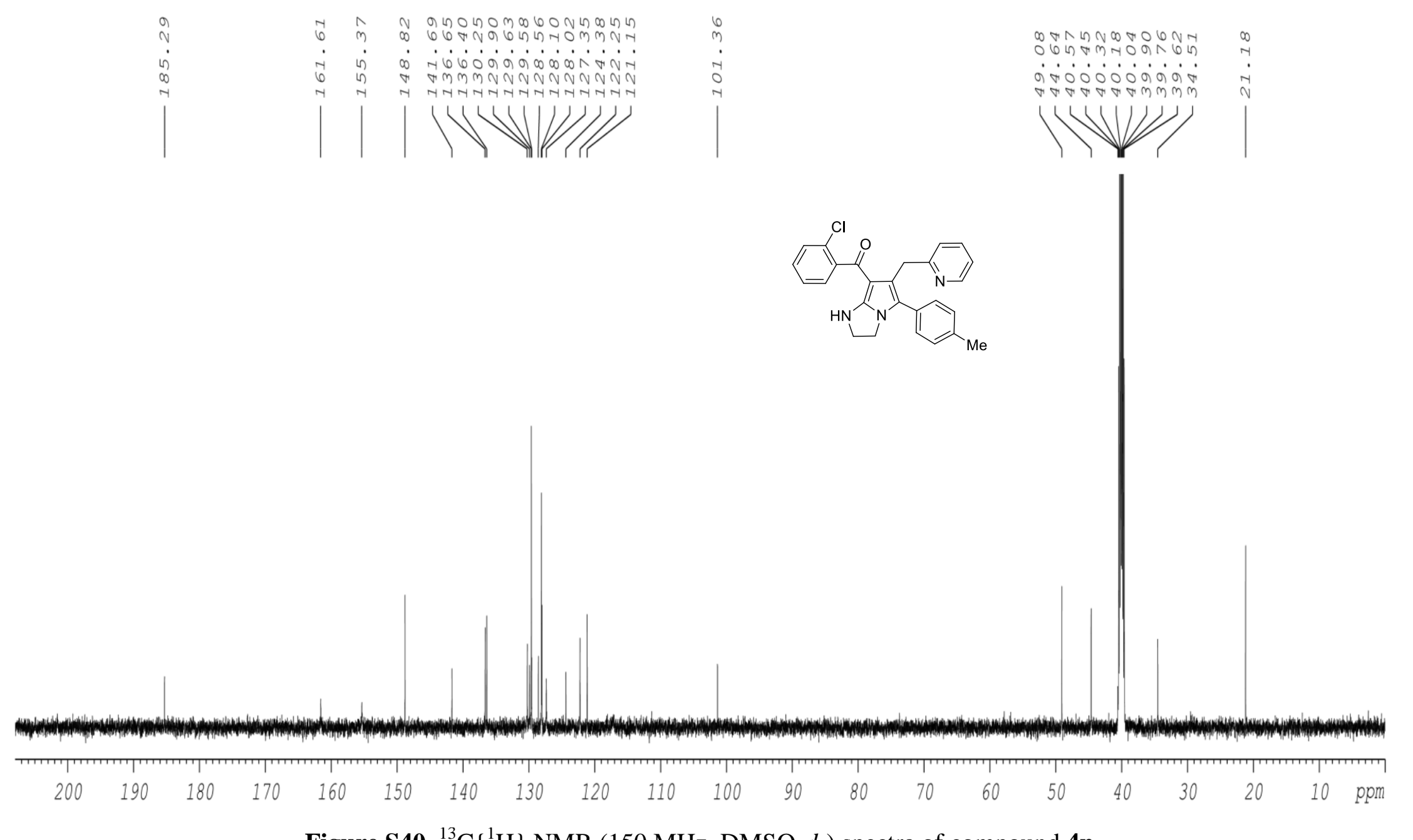

Figure S40. ${ }^{13} \mathrm{C}\left\{{ }^{1} \mathrm{H}\right\}$ NMR $\left(150 \mathrm{MHz}\right.$, DMSO- $\left.d_{6}\right)$ spectra of compound 4n 


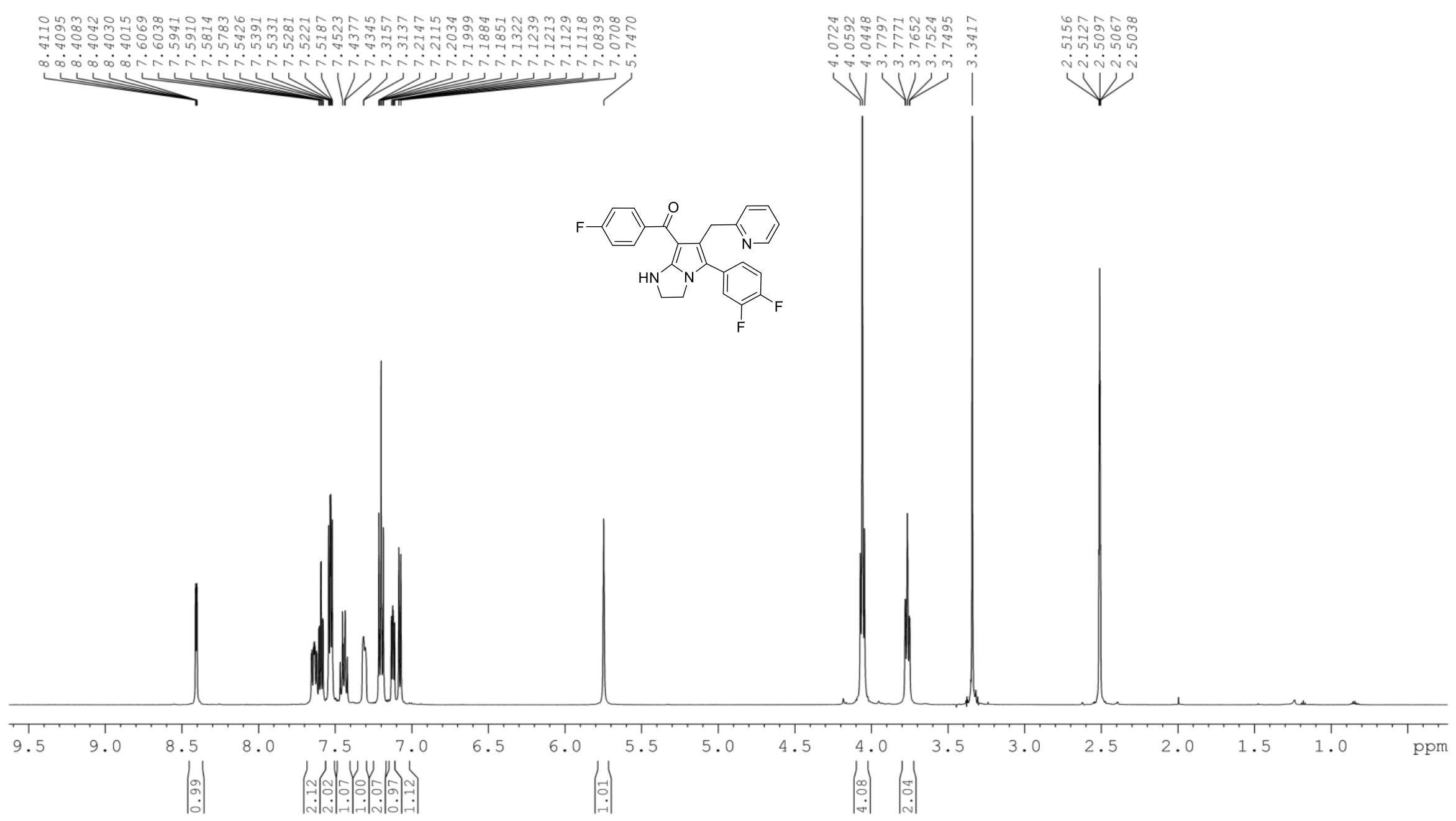

Figure S41. ${ }^{1} \mathrm{H}$ NMR (600 MHz, DMSO- $d_{6}$ ) spectra of compound 40 

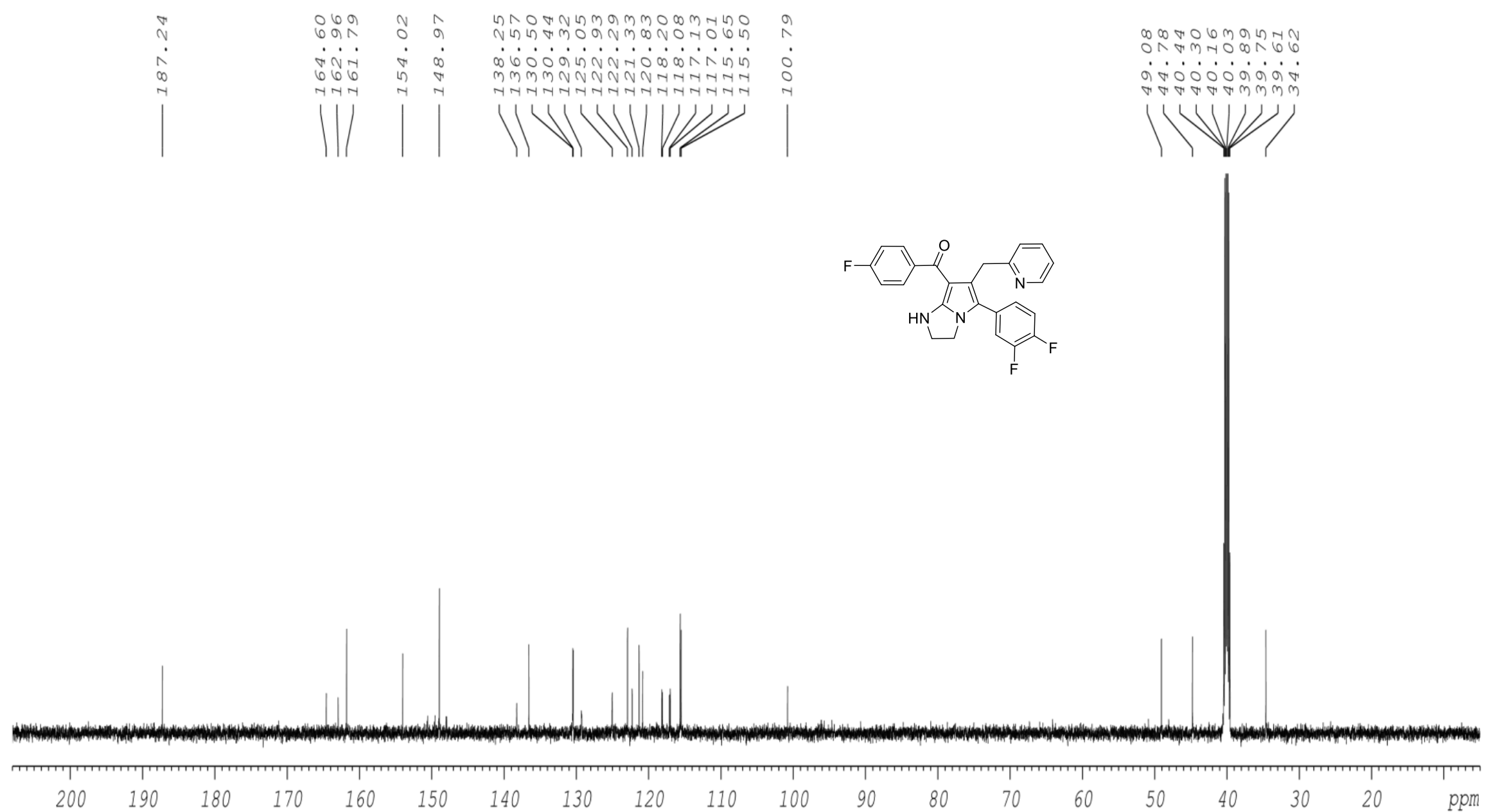

Figure S42. ${ }^{13} \mathrm{C}\left\{{ }^{1} \mathrm{H}\right\}$ NMR $\left(150 \mathrm{MHz}\right.$, DMSO- $\left.d_{6}\right)$ spectra of compound 40 
능유준

în

计

V v

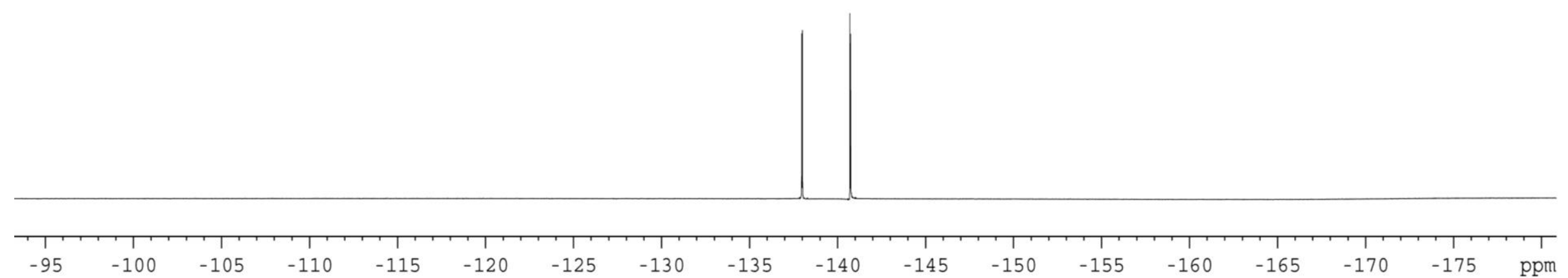

Figure S43. ${ }^{19} \mathrm{~F}$ NMR (470 MHz, DMSO- $d_{6}$ ) spectra of compound 40 


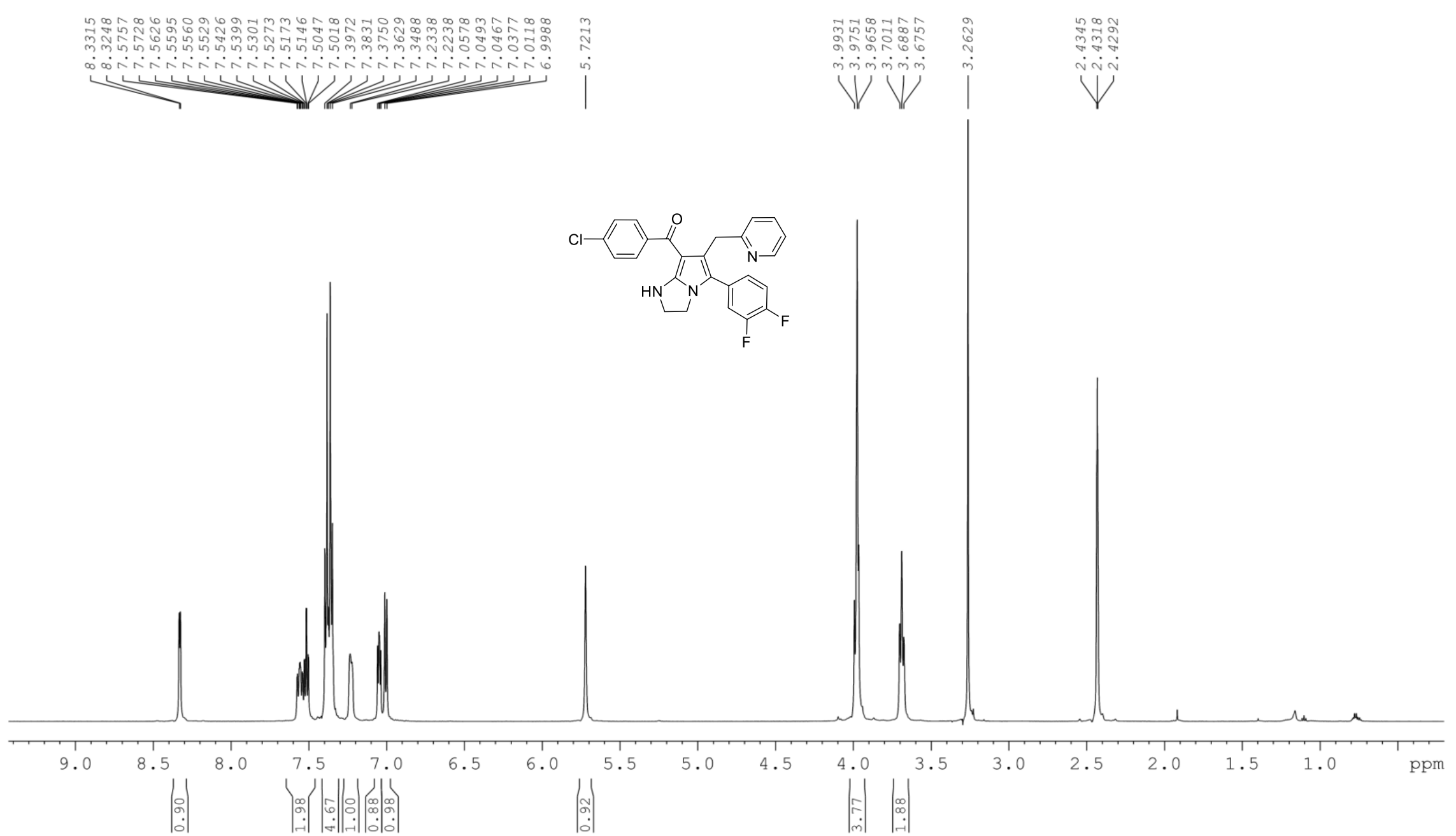

Figure S44. ${ }^{1} \mathrm{H}$ NMR (600 MHz, DMSO- $\left.d_{6}\right)$ spectra of compound $4 \mathbf{p}$ 


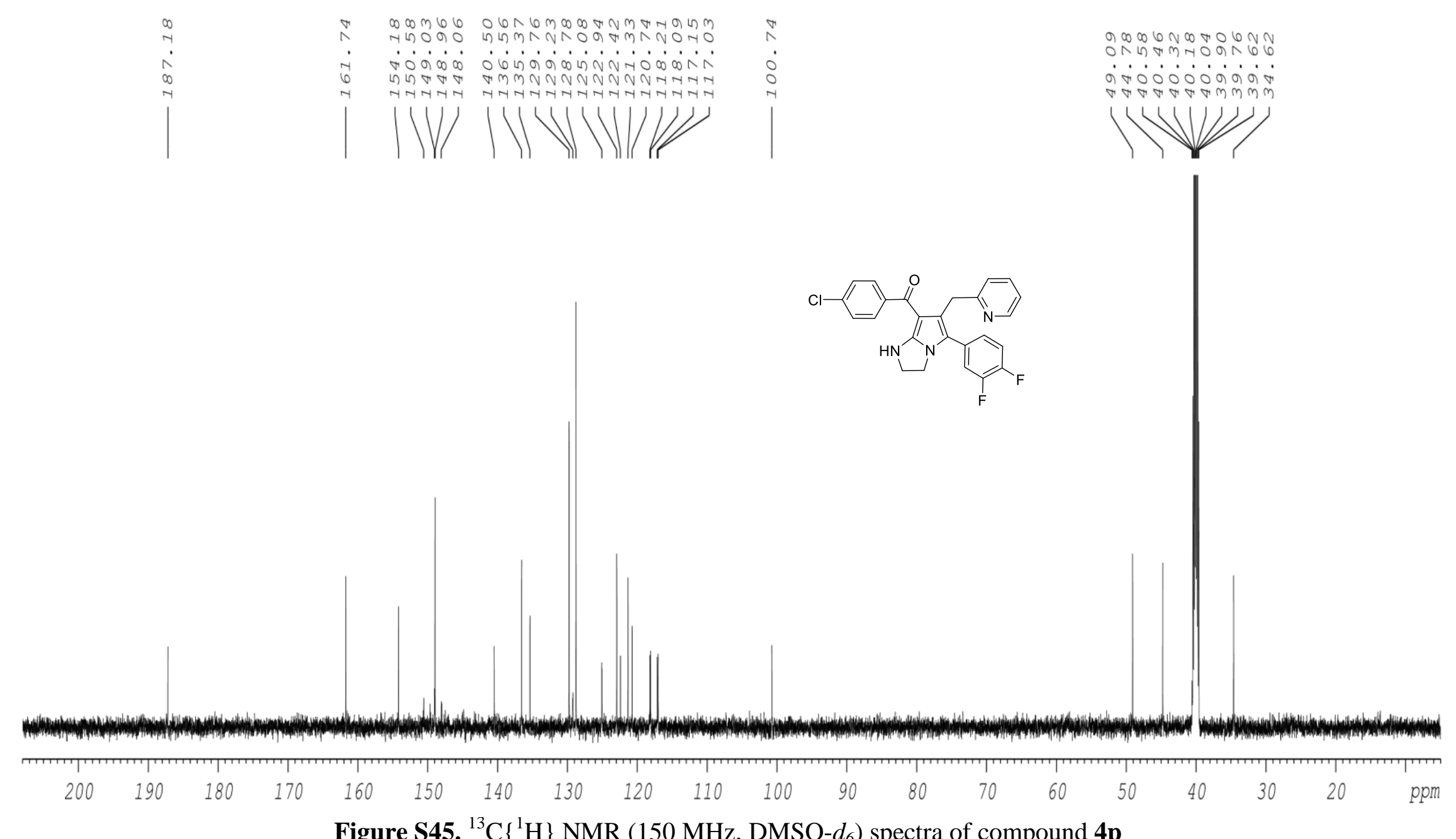

Figure S45. ${ }^{13} \mathrm{C}\left\{{ }^{1} \mathrm{H}\right\}$ NMR $\left(150 \mathrm{MHz}\right.$, DMSO- $\left.d_{6}\right)$ spectra of compound $\mathbf{4 p}$ 


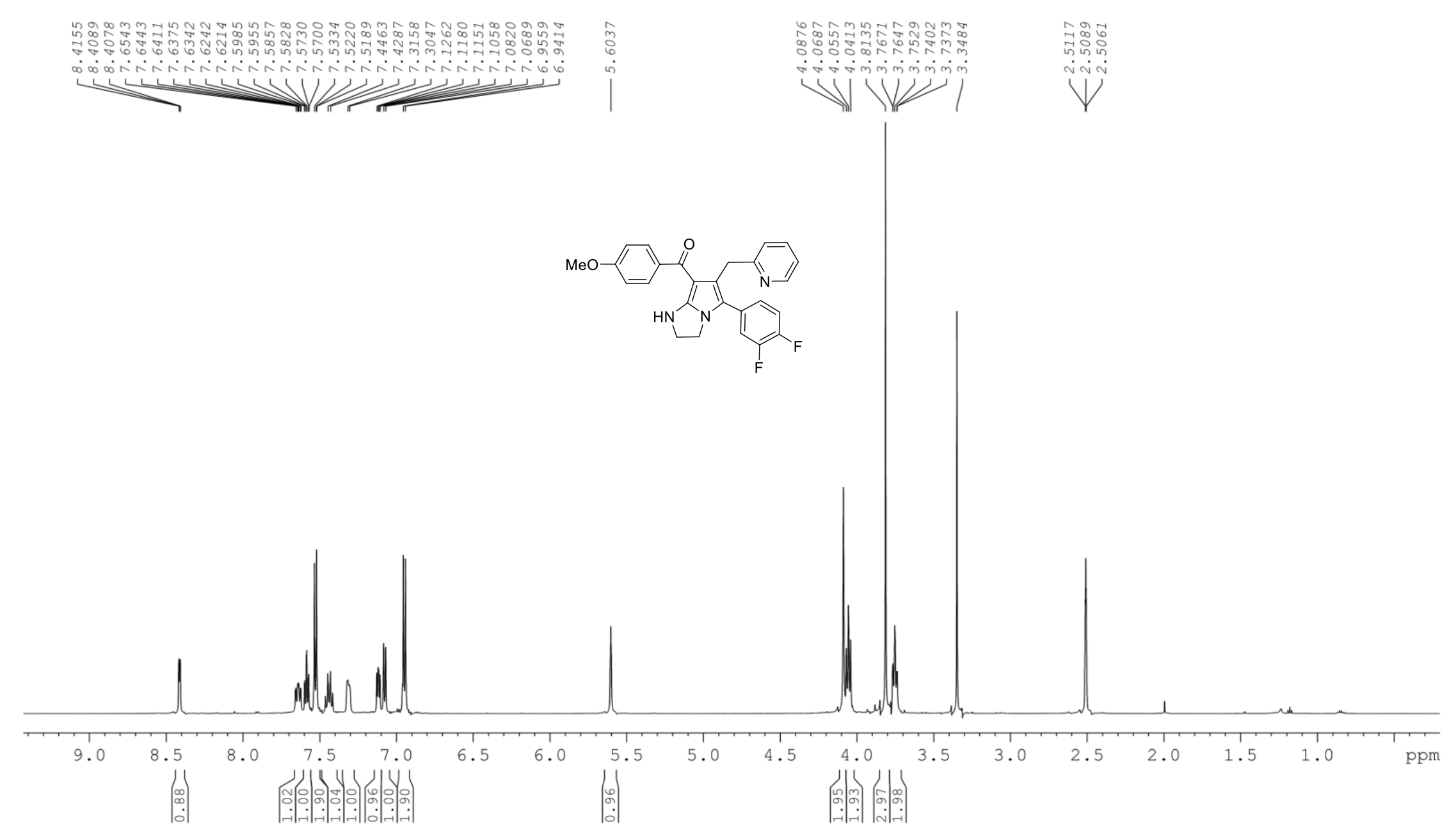

Figure S46. ${ }^{1} \mathrm{H}$ NMR (600 MHz, DMSO- $\left.d_{6}\right)$ spectra of compound $\mathbf{4 q}$ 


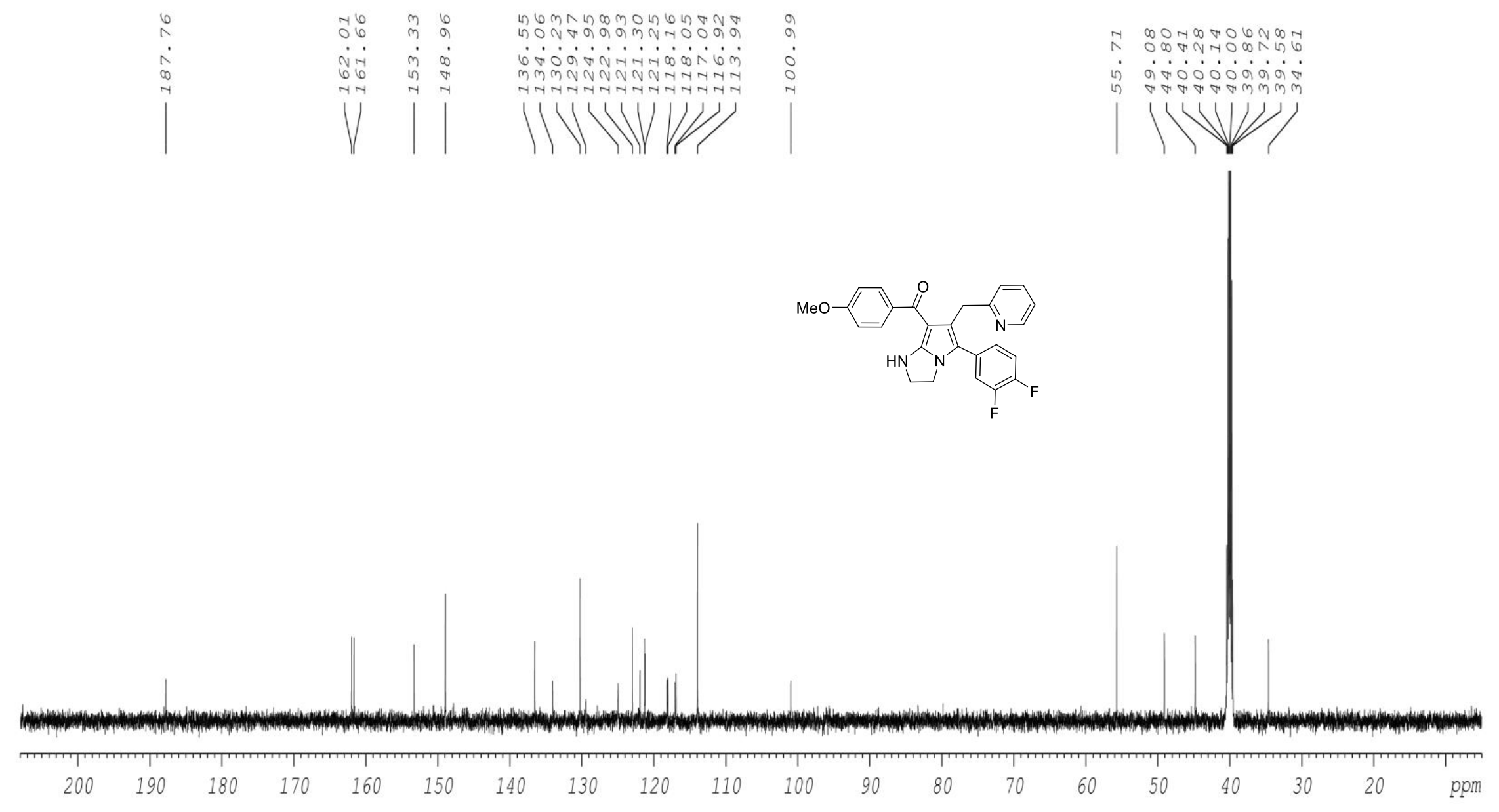

Figure S47. ${ }^{13} \mathrm{C}\left\{{ }^{1} \mathrm{H}\right\}$ NMR $\left(150 \mathrm{MHz}, \mathrm{DMSO}-d_{6}\right)$ spectra of compound $\mathbf{4 q}$ 

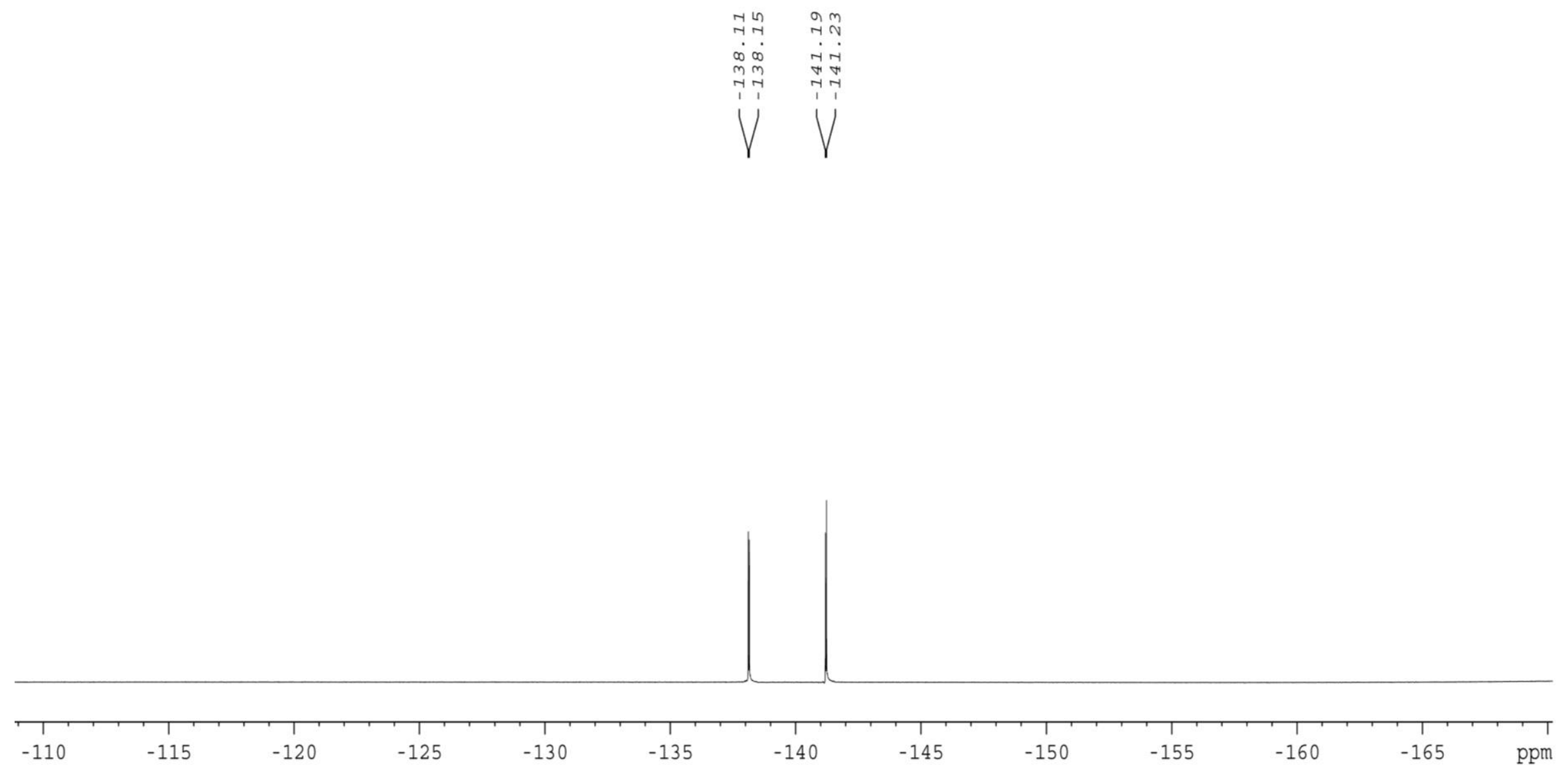

Figure S48. ${ }^{19}$ F NMR (470 MHz, DMSO- $d_{6}$ ) spectra of compound $4 \mathbf{q}$ 


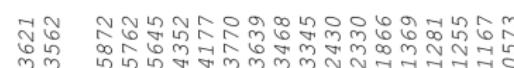

w

$\checkmark+N V I T$
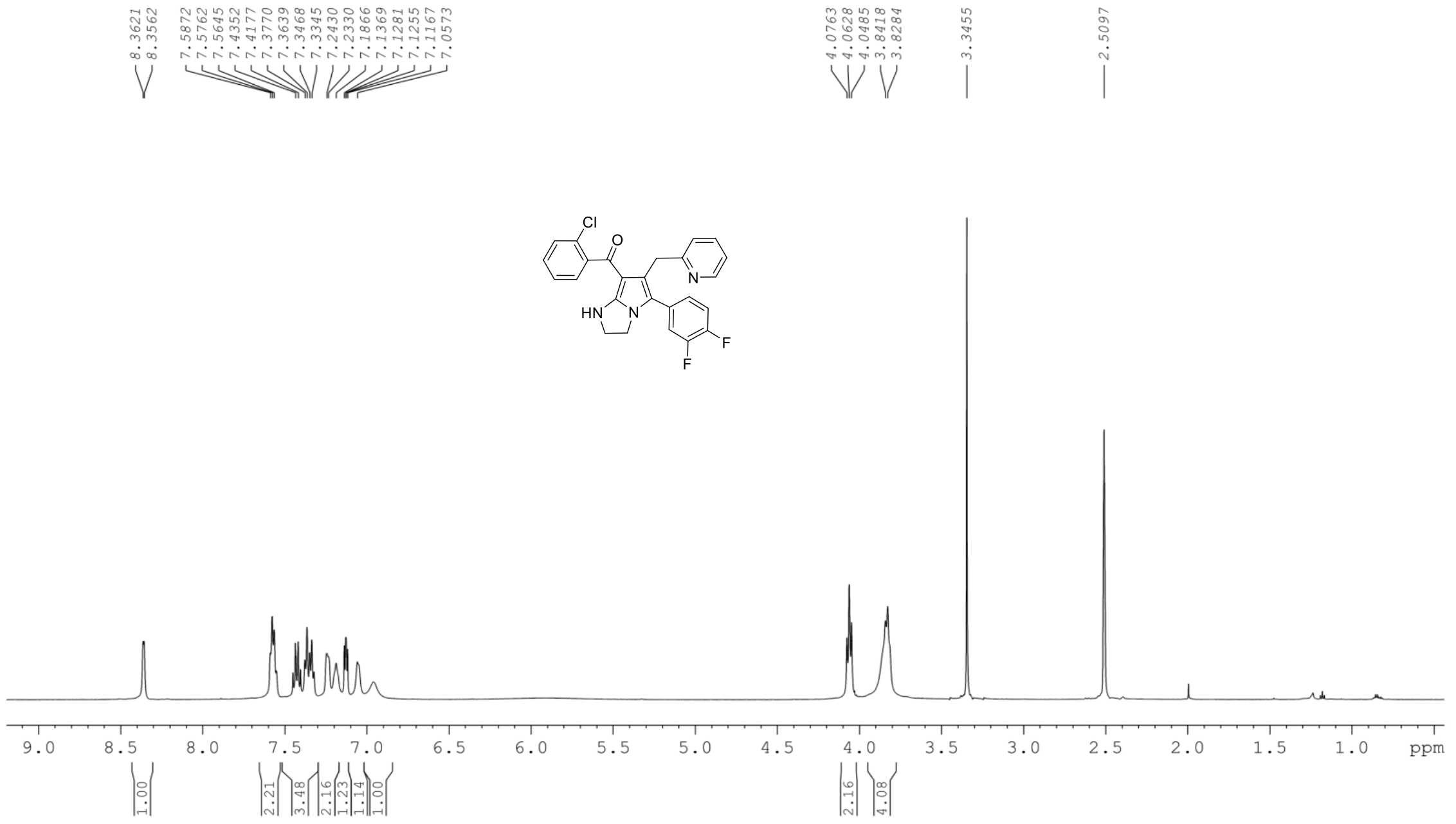

Figure S49. ${ }^{1} \mathrm{H}$ NMR $\left(600 \mathrm{MHz}, \mathrm{DMSO}-d_{6}\right)$ spectra of compound $4 \mathbf{r}$ 


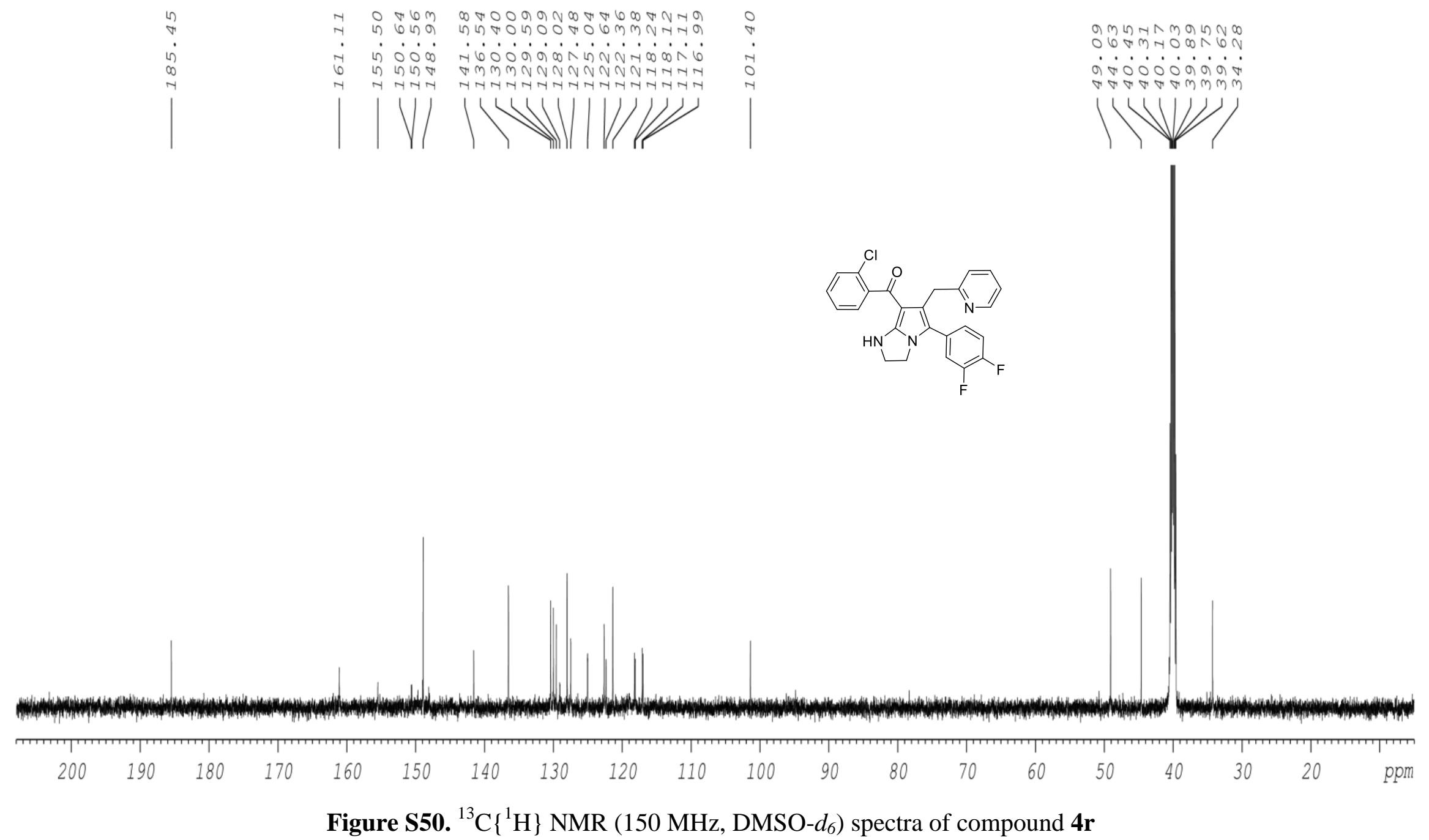



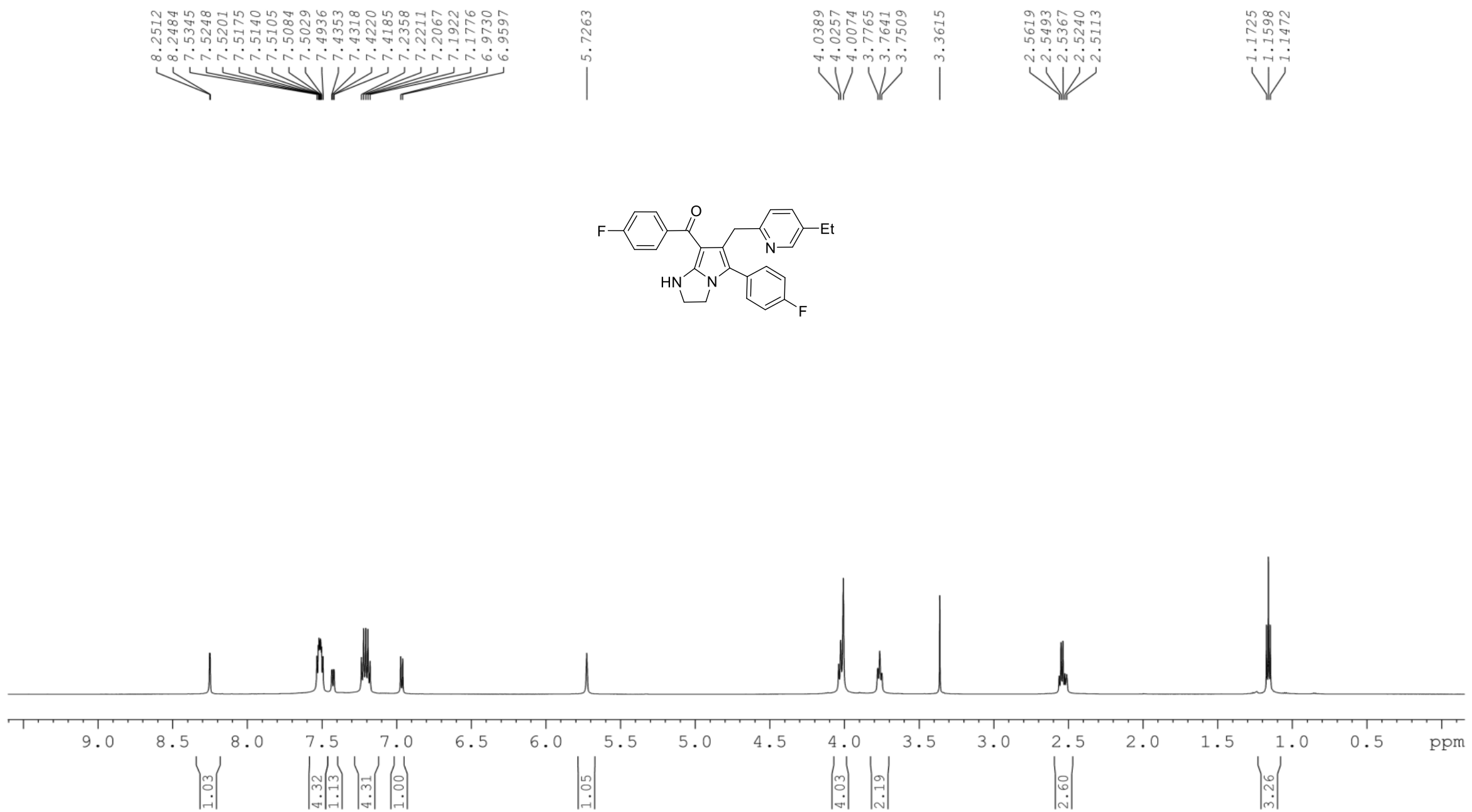

Figure S51. ${ }^{1} \mathrm{H}$ NMR (600 MHz, DMSO- $\left.d_{6}\right)$ spectra of compound 4s 


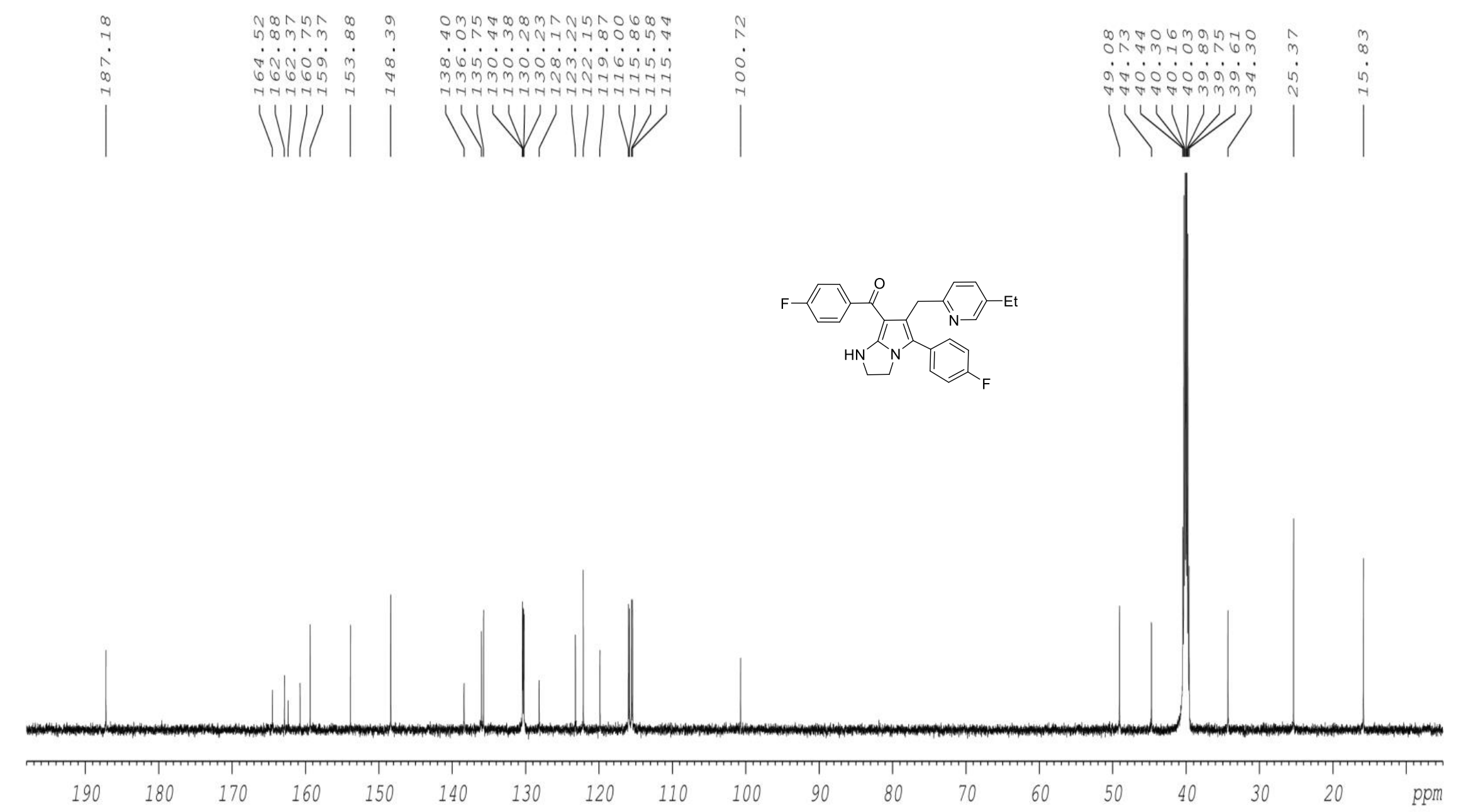

Figure S52. ${ }^{13} \mathrm{C}\left\{{ }^{1} \mathrm{H}\right\}$ NMR $\left(150 \mathrm{MHz}\right.$, DMSO- $\left.d_{6}\right)$ spectra of compound $4 \mathbf{s}$ 

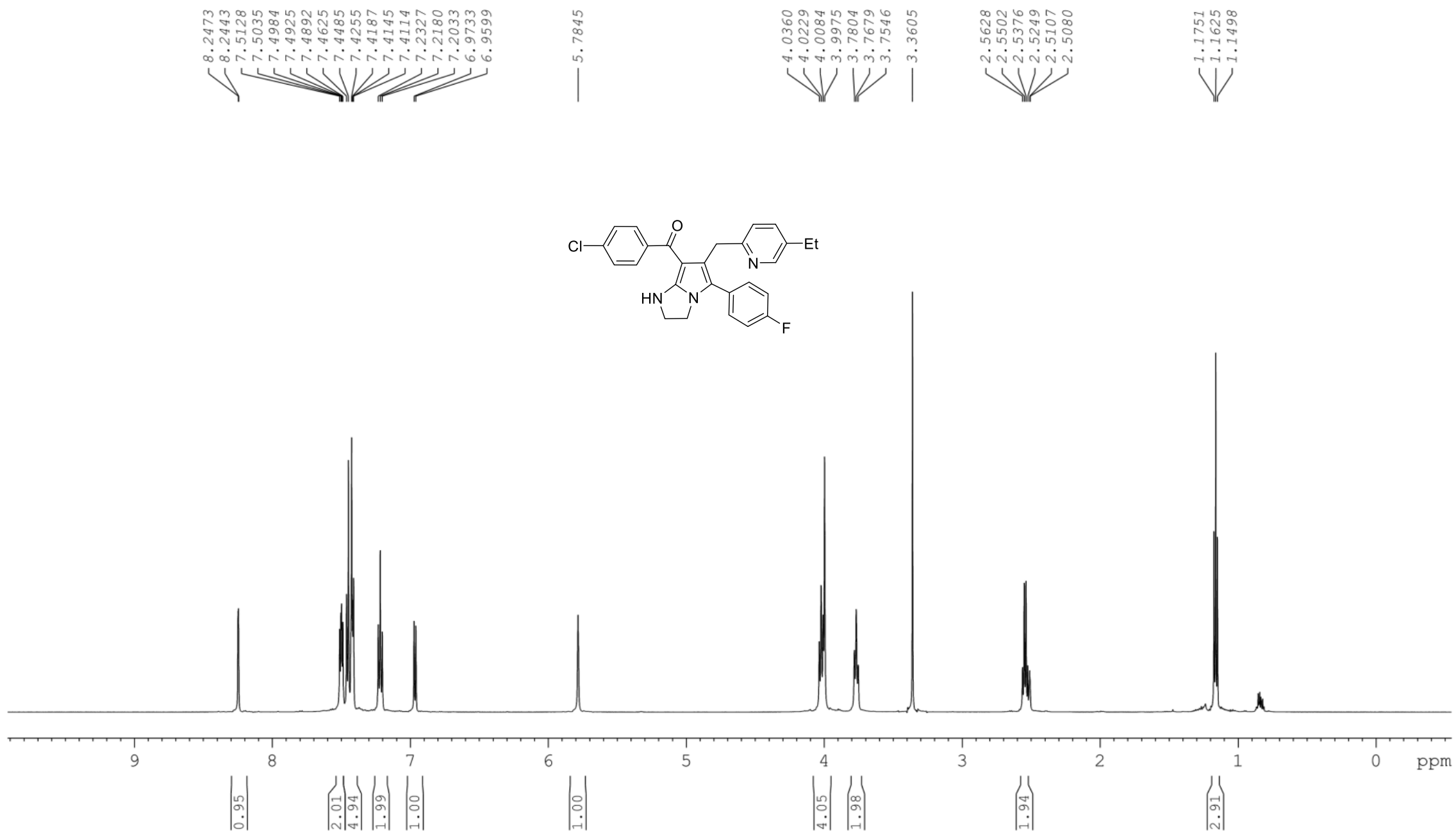

Figure S53. ${ }^{1} \mathrm{H}$ NMR (600 MHz, DMSO- $\left.d_{6}\right)$ spectra of compound $4 \mathbf{t}$ 

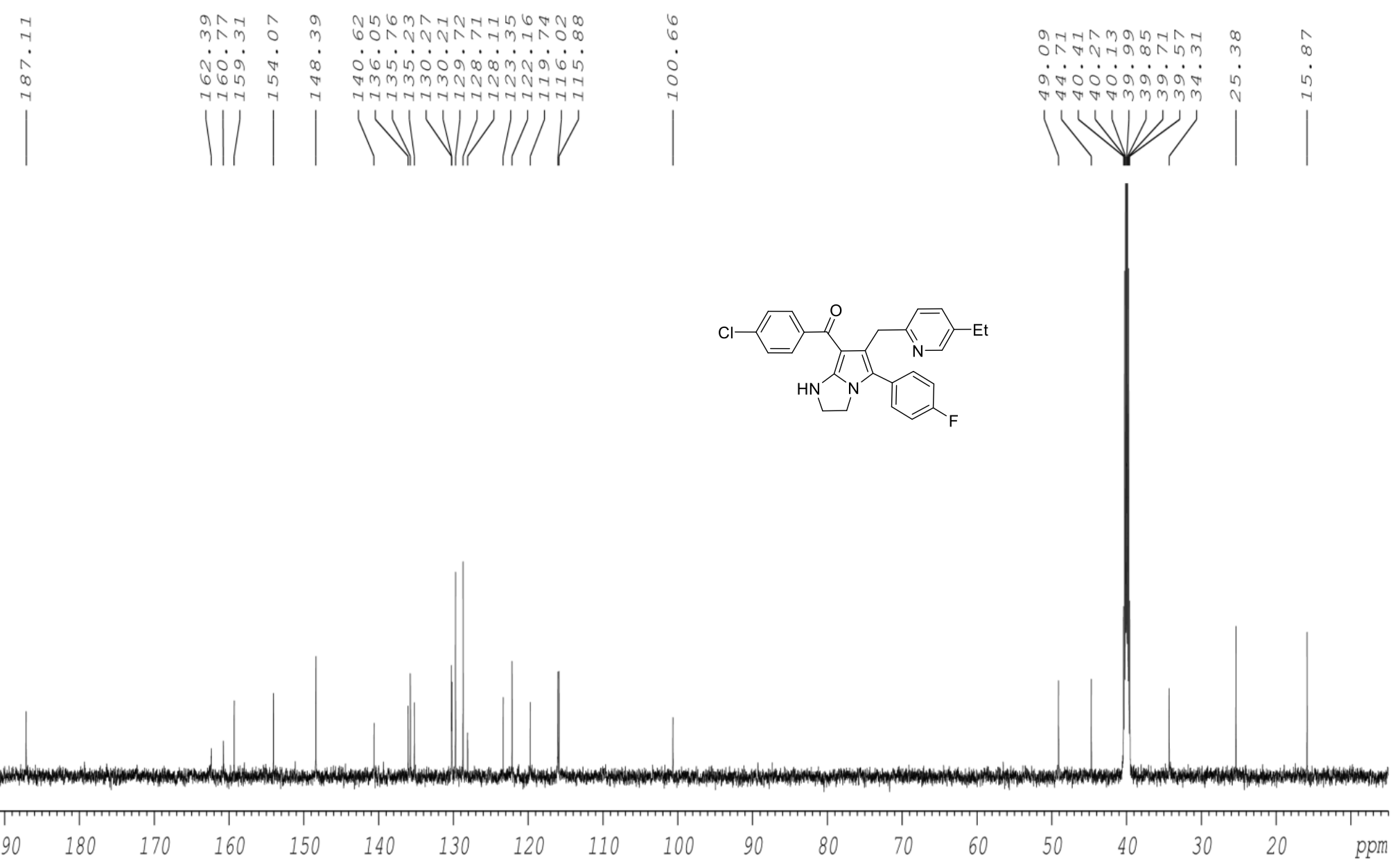

$16 m$ mom

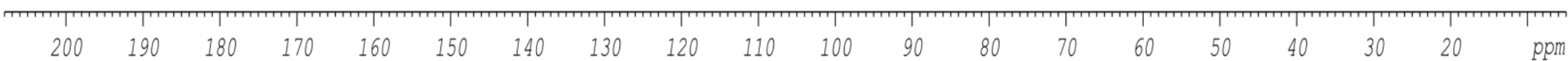

Figure S54. ${ }^{13} \mathrm{C}\left\{{ }^{1} \mathrm{H}\right\}$ NMR $\left(150 \mathrm{MHz}, \mathrm{DMSO}-d_{6}\right)$ spectra of compound $\mathbf{4 t}$ 


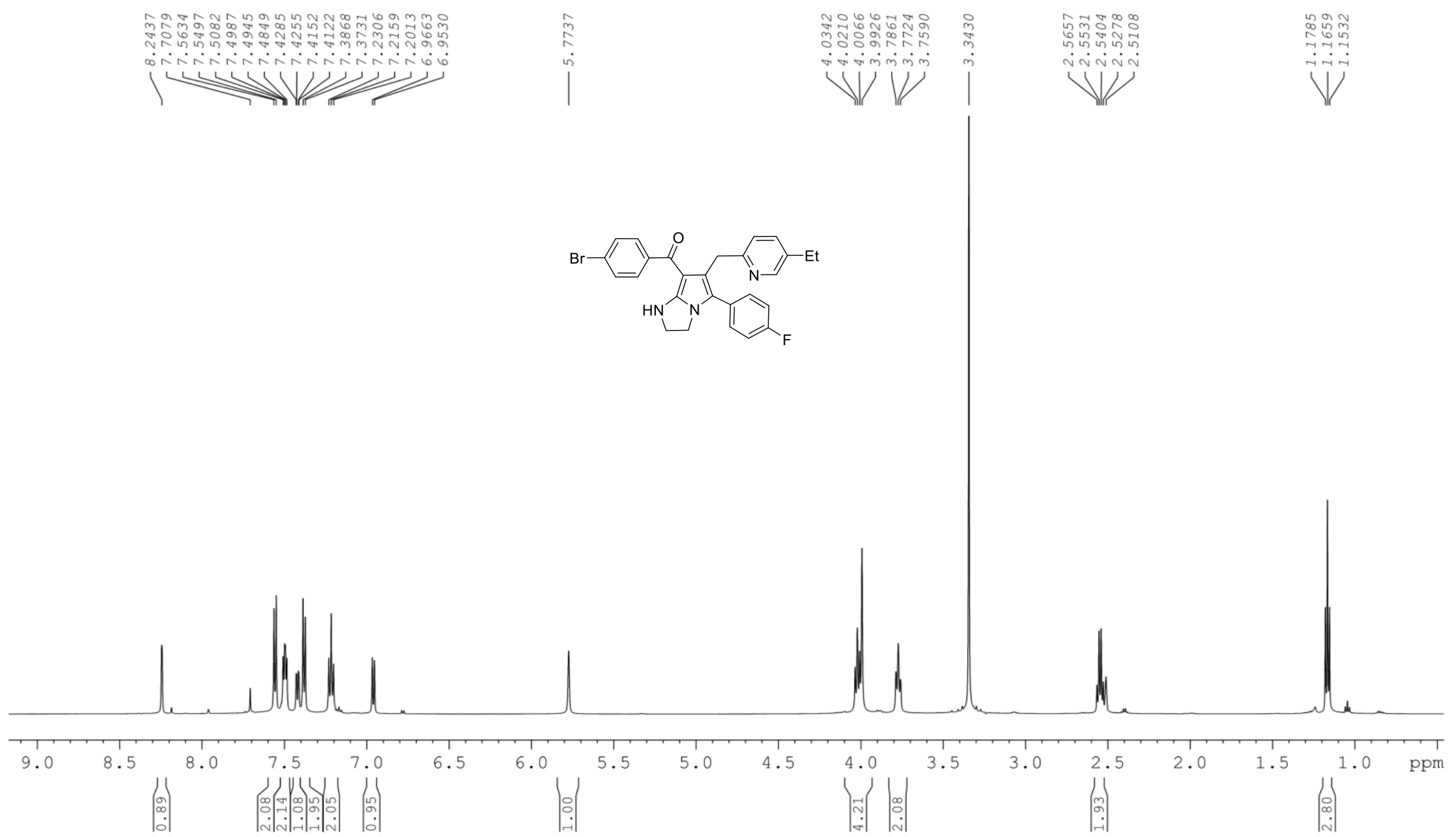

Figure S55. ${ }^{1} \mathrm{H}$ NMR (600 MHz, DMSO- $\left.d_{6}\right)$ spectra of compound $4 \mathbf{u}$ 


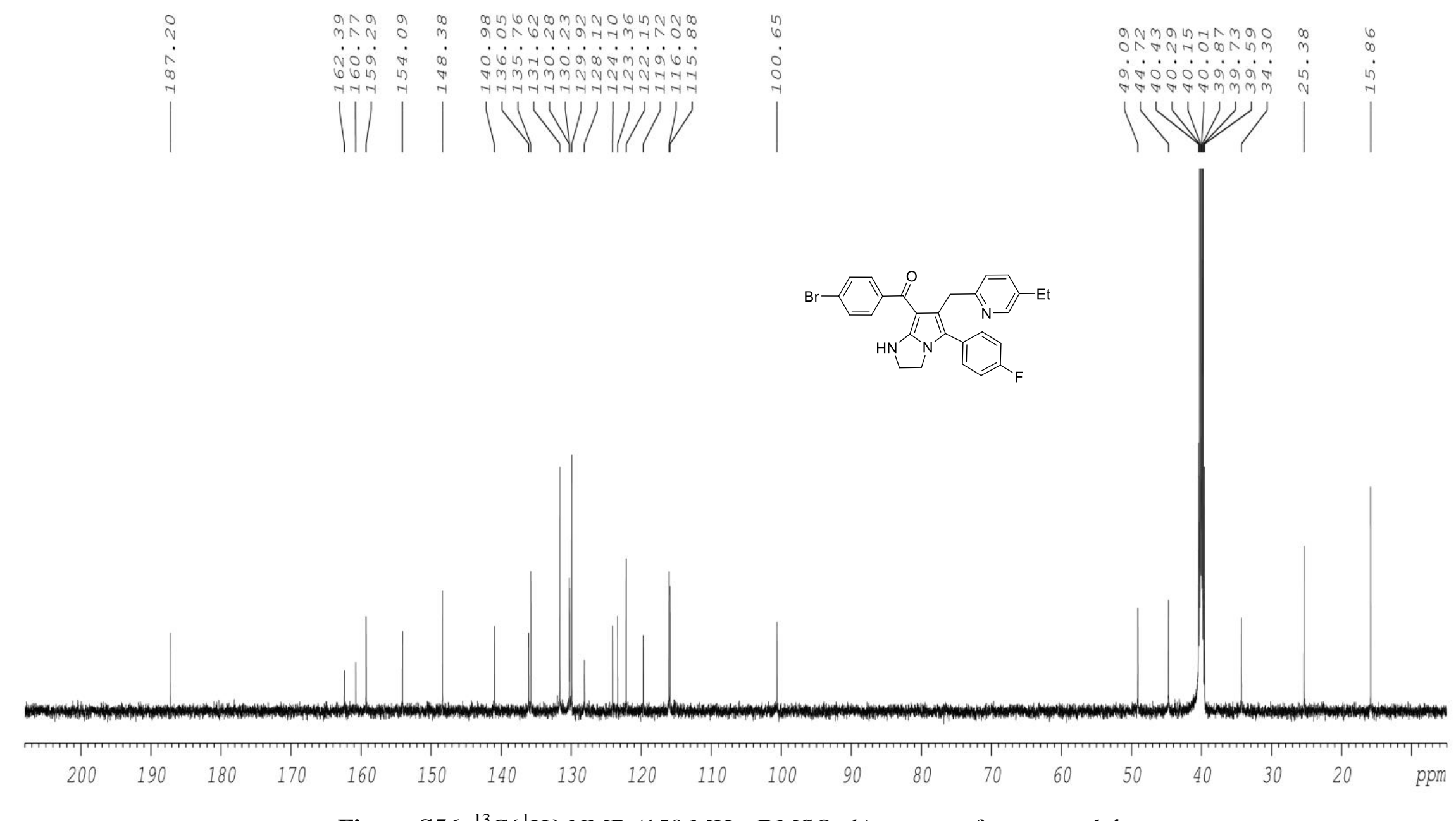

Figure S56. ${ }^{13} \mathrm{C}\left\{{ }^{1} \mathrm{H}\right\}$ NMR $\left(150 \mathrm{MHz}\right.$, DMSO- $\left.d_{6}\right)$ spectra of compound $\mathbf{4 u}$ 

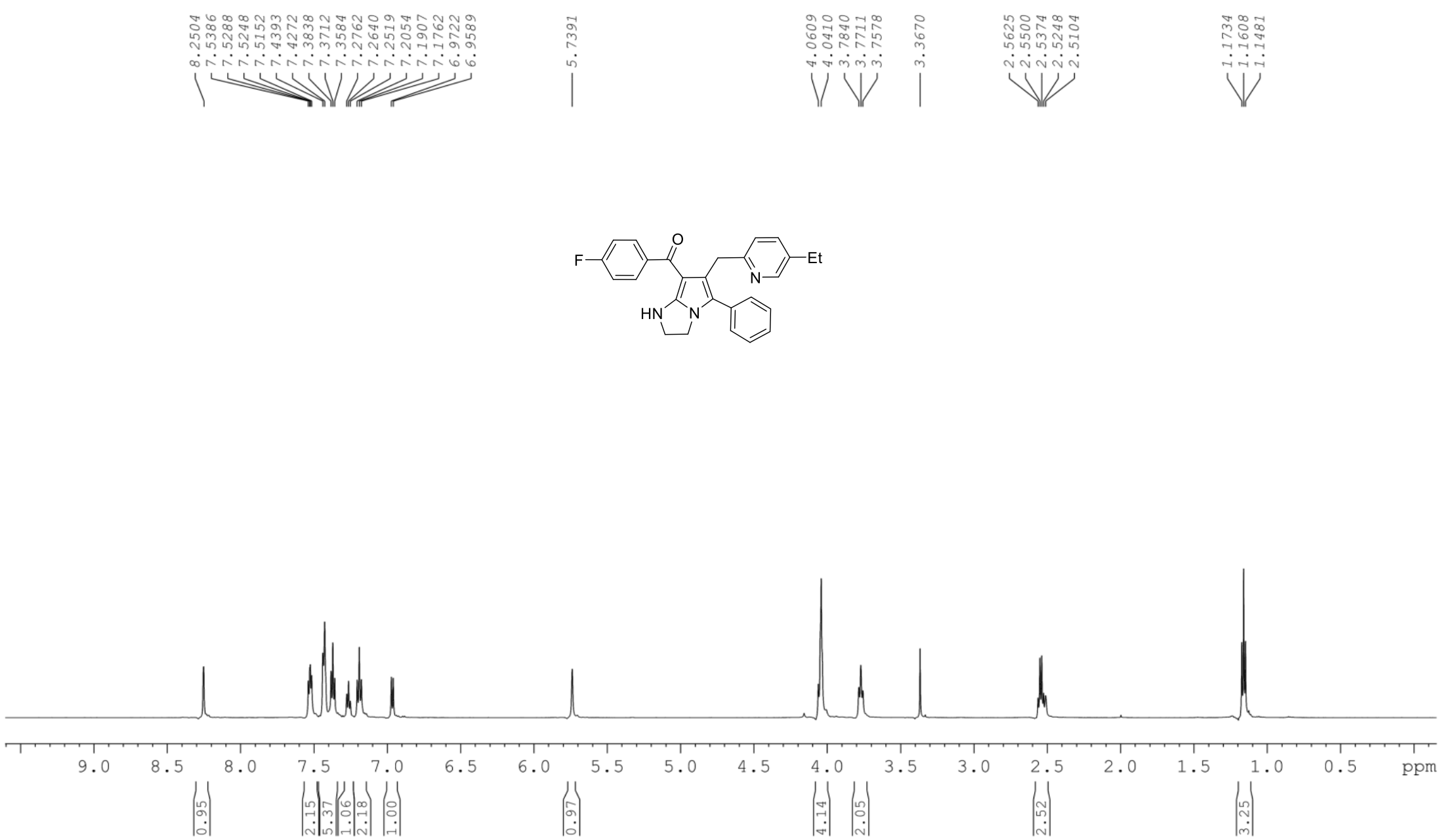

Figure S57. ${ }^{1} \mathrm{H}$ NMR (600 MHz, DMSO- $\left.d_{6}\right)$ spectra of compound $\mathbf{4 v}$ 


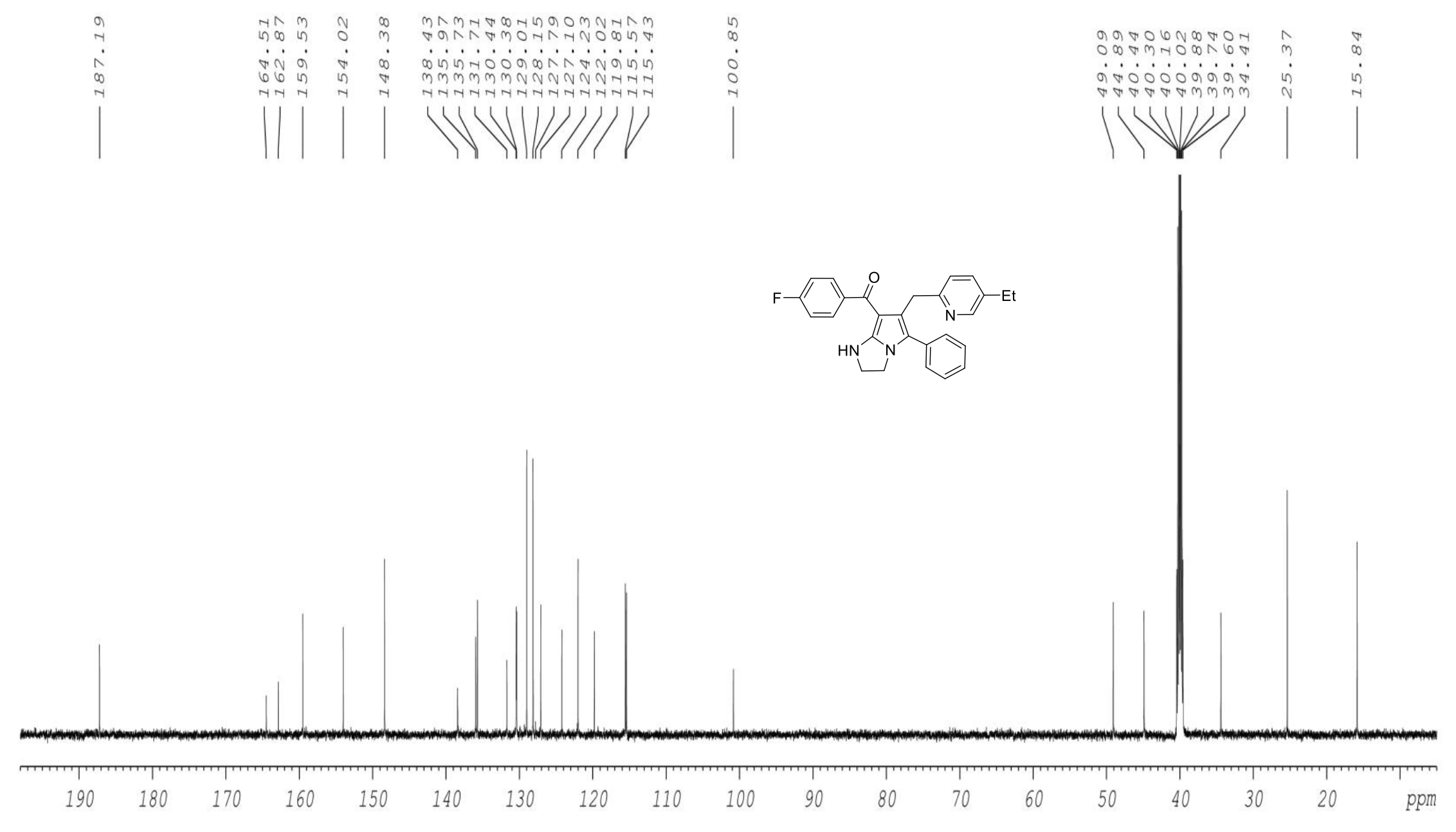

Figure S58. ${ }^{13} \mathrm{C}\left\{{ }^{1} \mathrm{H}\right\}$ NMR $\left(150 \mathrm{MHz}\right.$, DMSO- $\left.d_{6}\right)$ spectra of compound $\mathbf{4 v}$ 

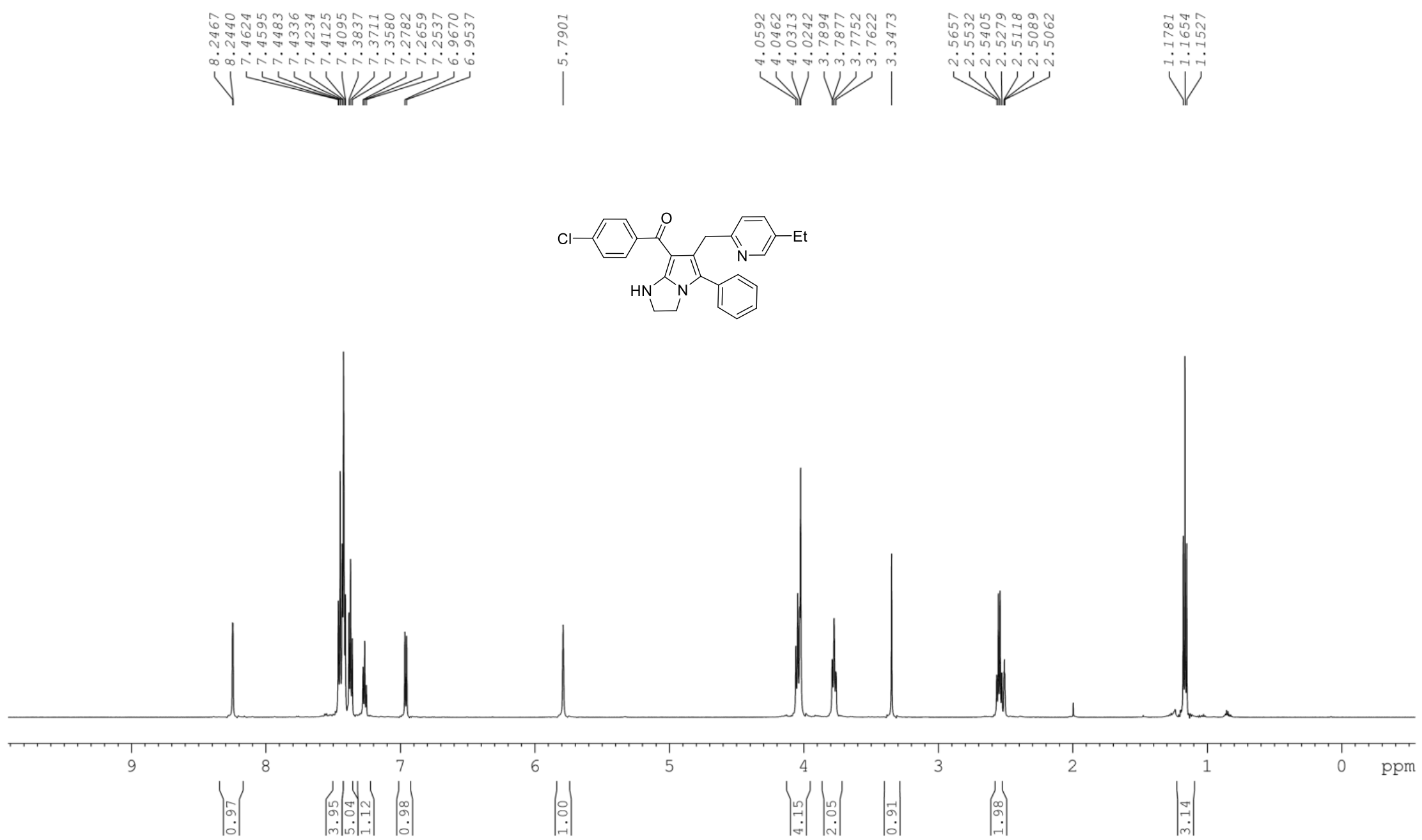

Figure S59. ${ }^{1} \mathrm{H}$ NMR (600 MHz, DMSO- $\left.d_{6}\right)$ spectra of compound $4 \mathbf{w}$ 


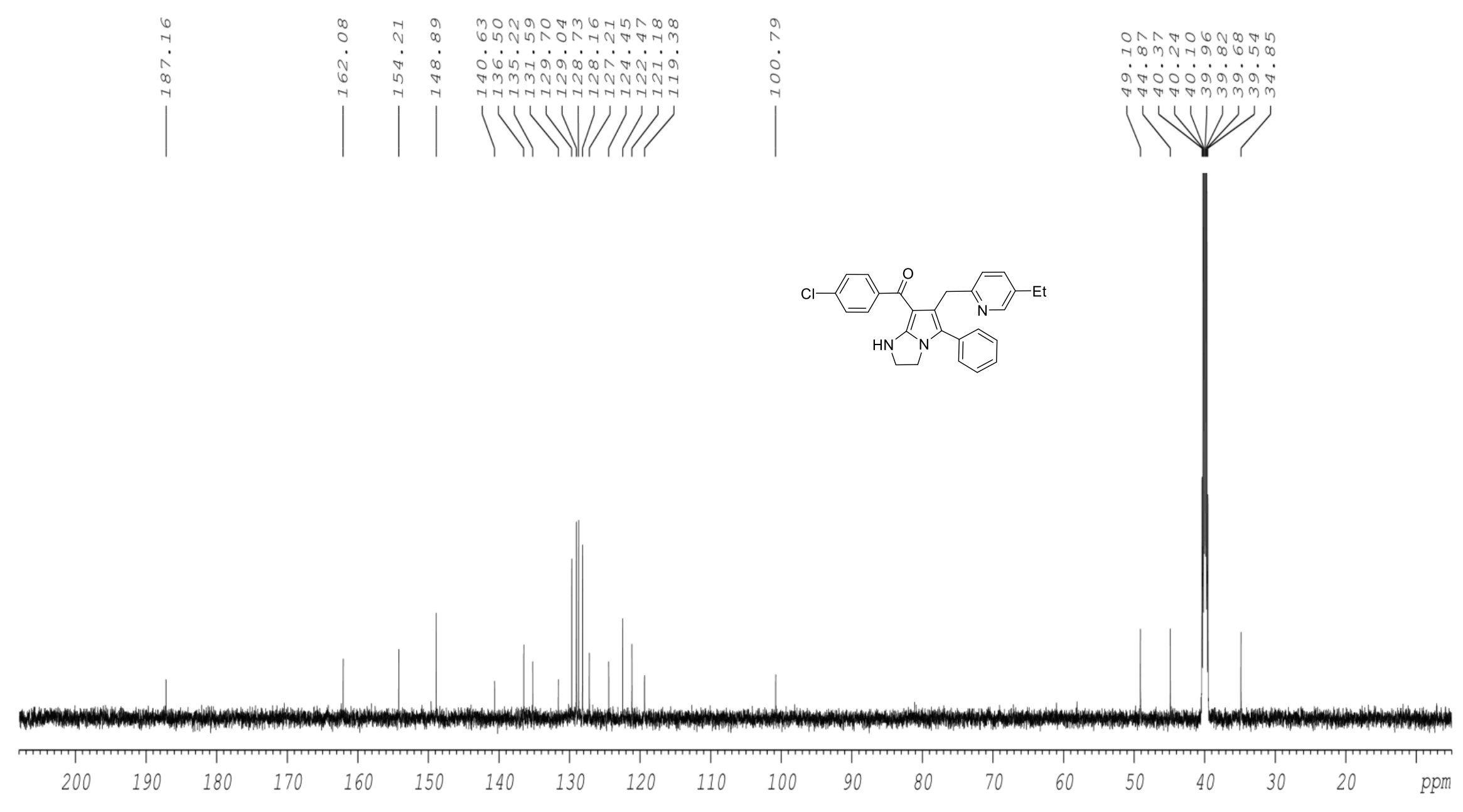

Figure S60. ${ }^{13} \mathrm{C}\left\{{ }^{1} \mathrm{H}\right\}$ NMR $\left(150 \mathrm{MHz}, \mathrm{DMSO}-d_{6}\right)$ spectra of compound $\mathbf{4 w}$ 

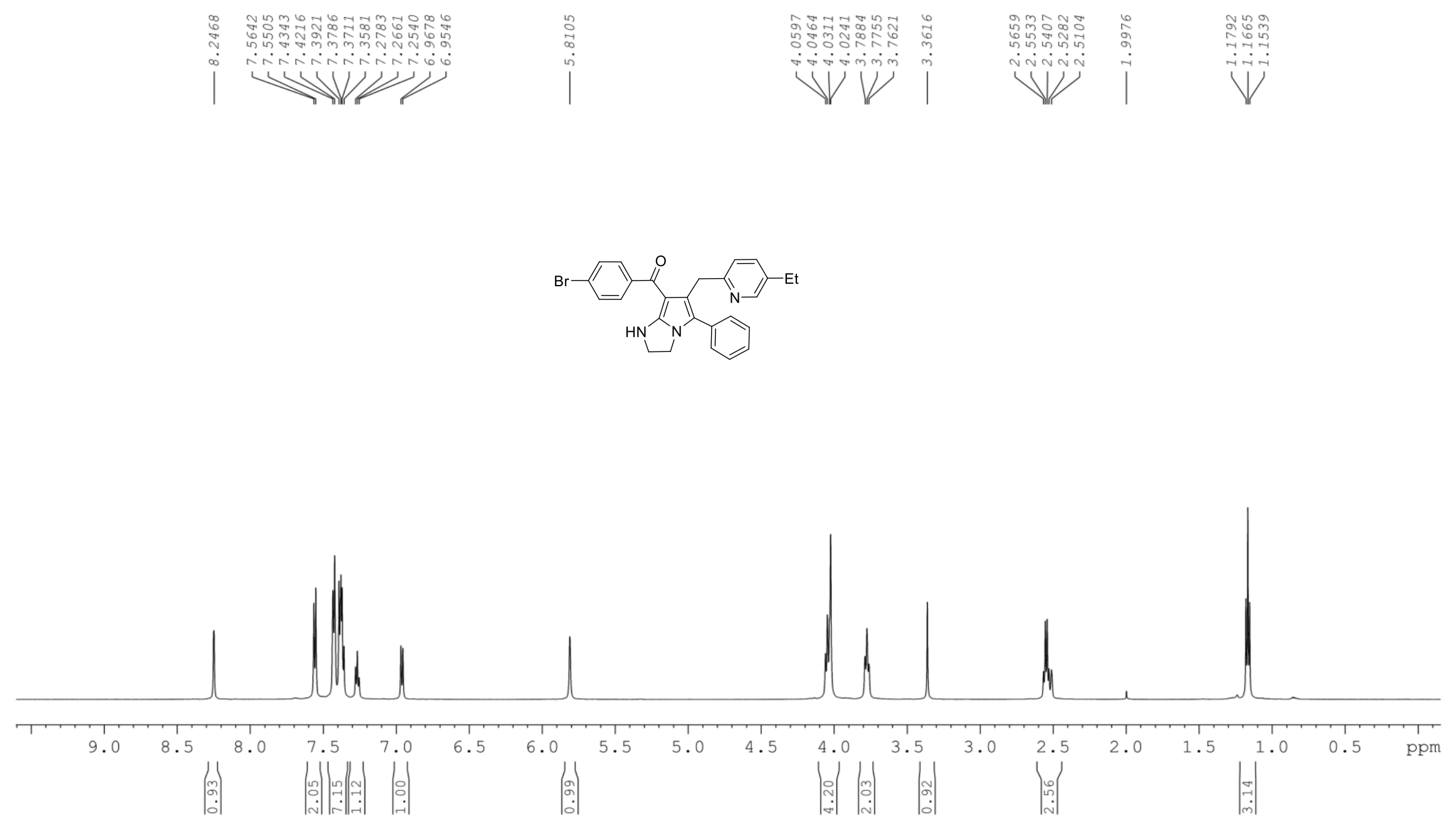

Figure S61. ${ }^{1} \mathrm{H}$ NMR (600 MHz, DMSO- $\left.d_{6}\right)$ spectra of compound $\mathbf{4 x}$ 


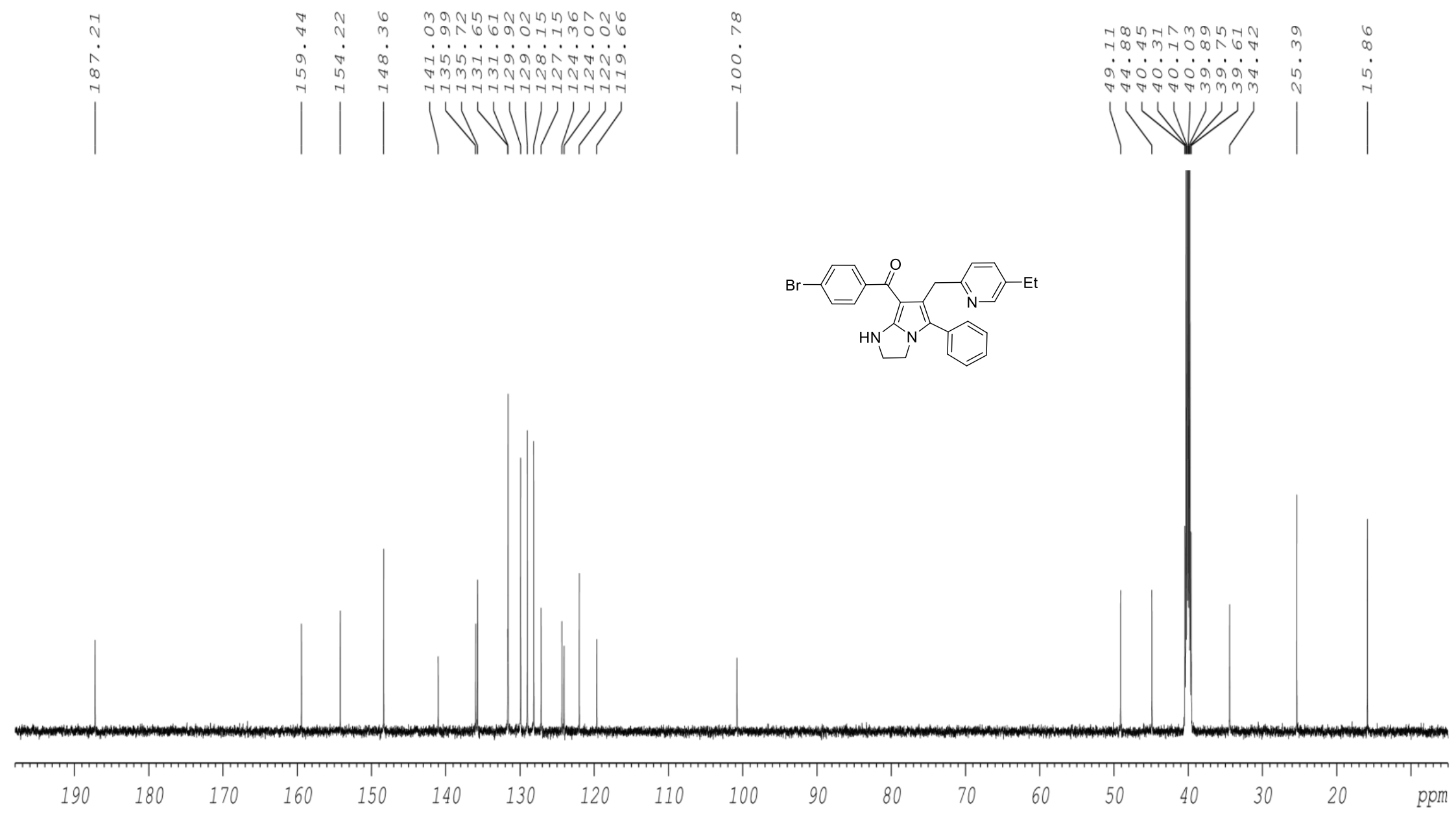

Figure S62. ${ }^{13} \mathrm{C}\left\{{ }^{1} \mathrm{H}\right\}$ NMR (150 MHz, DMSO- $\left.d_{6}\right)$ spectra of compound $\mathbf{4 x}$ 

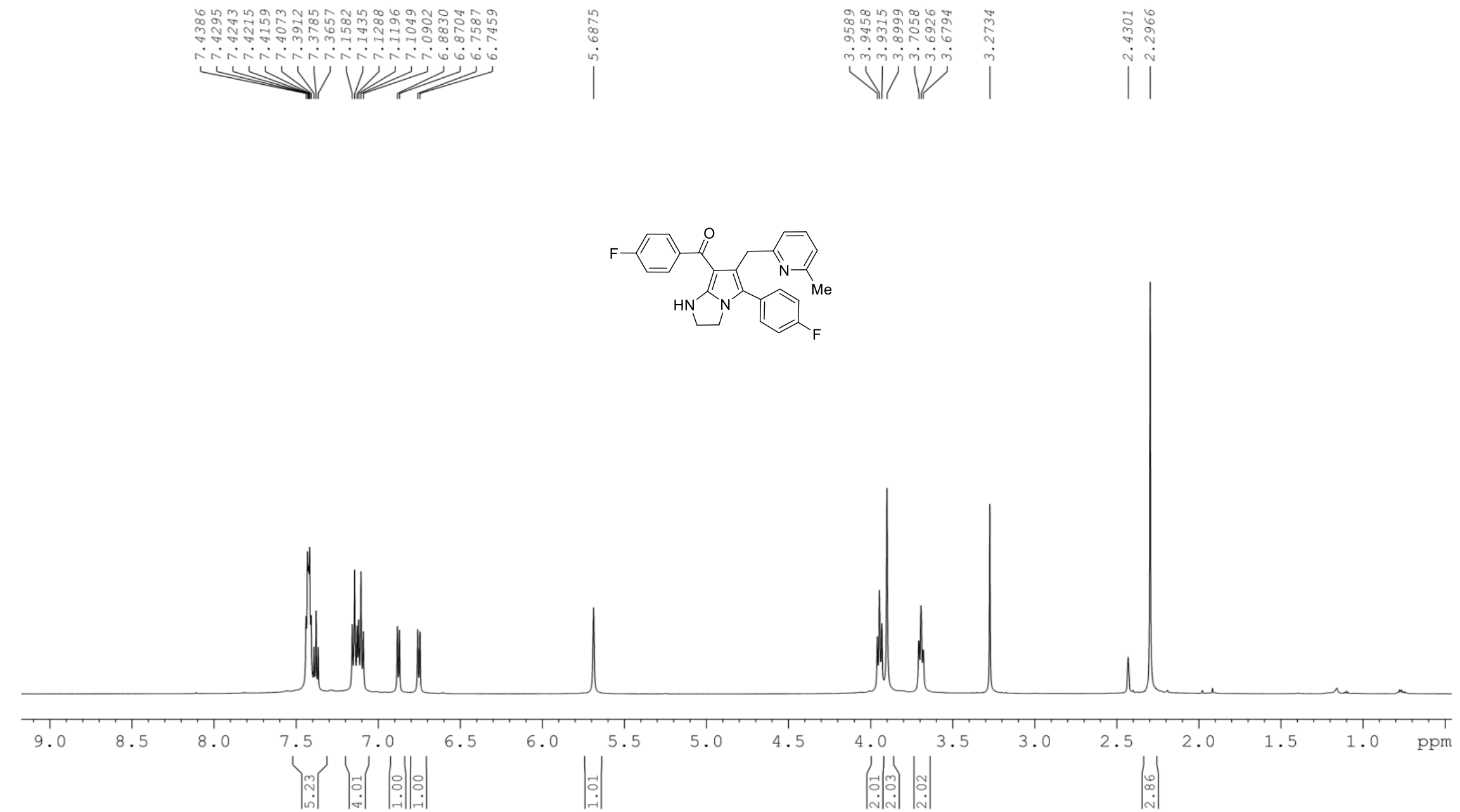

Figure S63. ${ }^{1} \mathrm{H}$ NMR (600 MHz, DMSO- $\left.d_{6}\right)$ spectra of compound $\mathbf{4 y}$ 


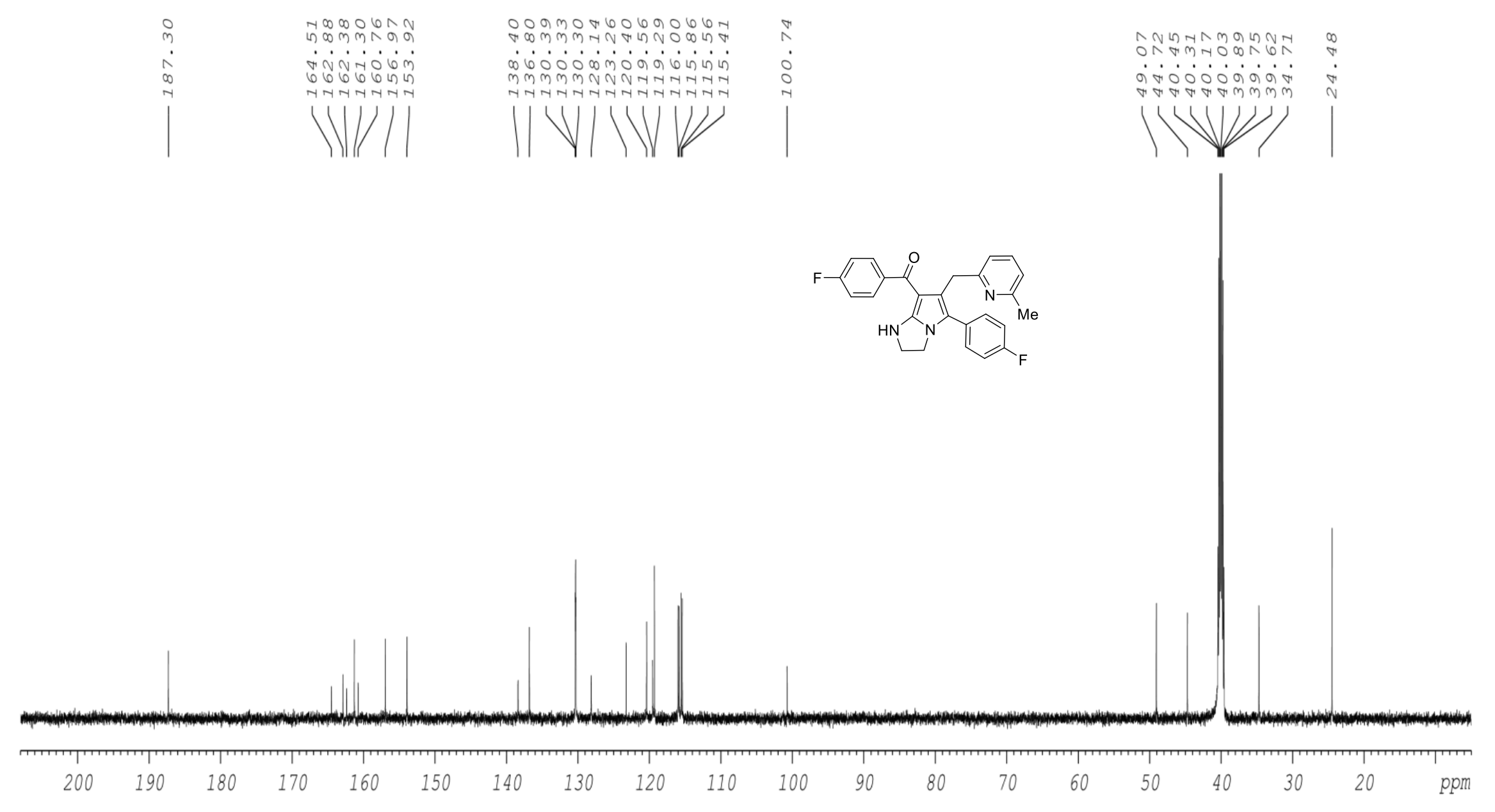

Figure S64. ${ }^{13} \mathrm{C}\left\{{ }^{1} \mathrm{H}\right\}$ NMR $\left(150 \mathrm{MHz}\right.$, DMSO- $\left.d_{6}\right)$ spectra of compound $\mathbf{4 y}$ 

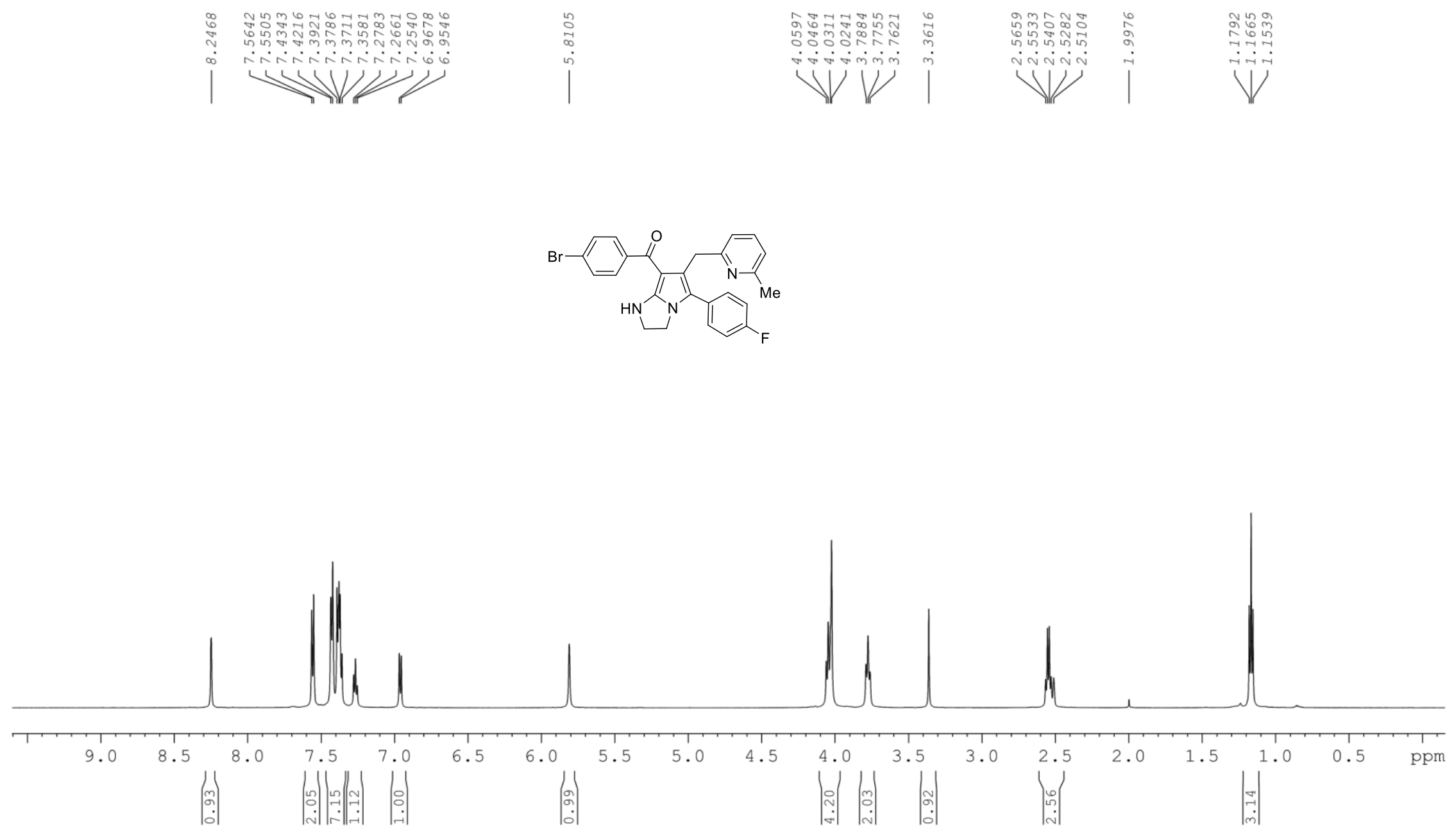

Figure S65. ${ }^{1} \mathrm{H}$ NMR $\left(600 \mathrm{MHz}, \mathrm{DMSO}-d_{6}\right)$ spectra of compound $\mathbf{4 z}$ 


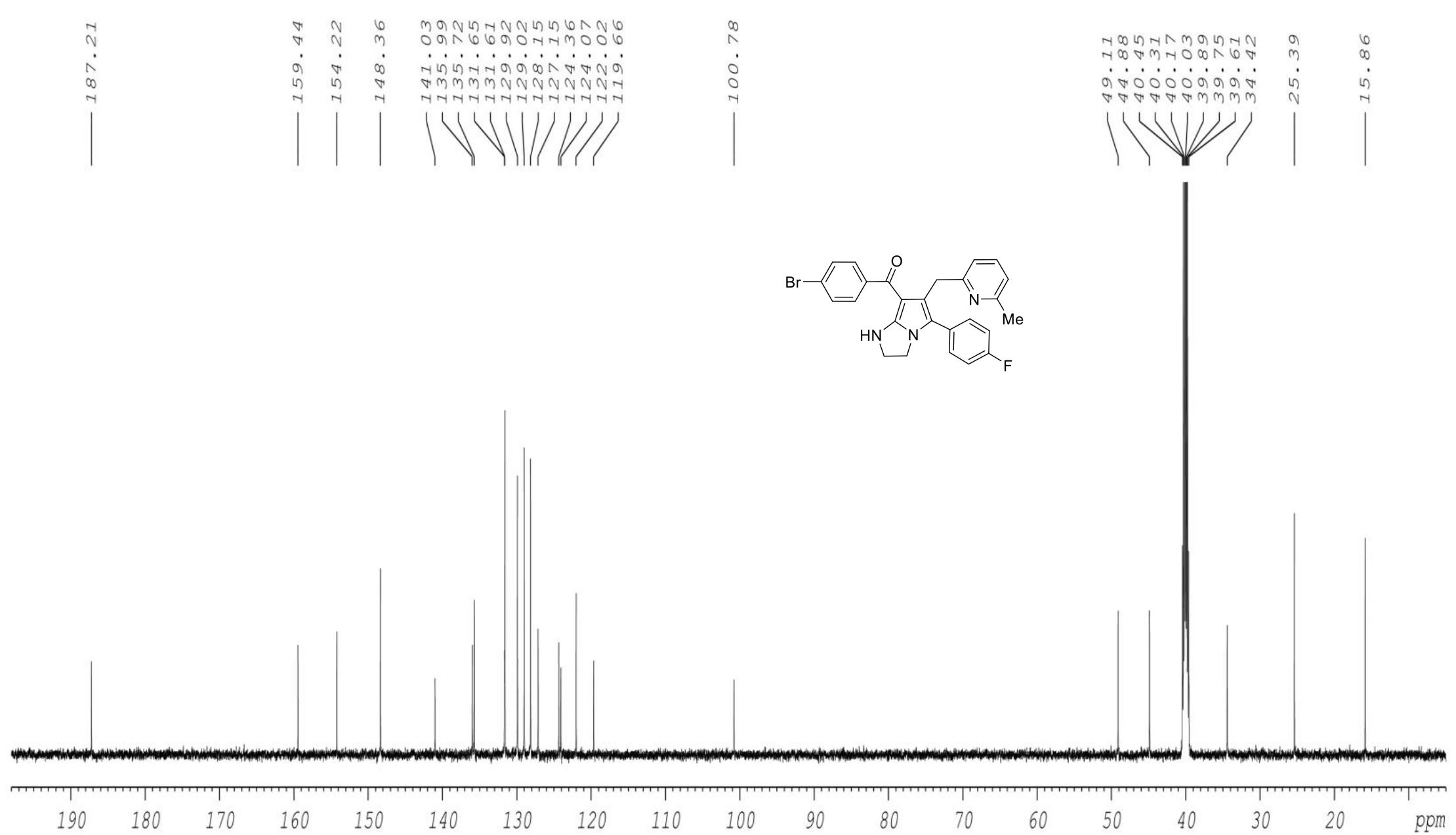

Figure S66. ${ }^{13} \mathrm{C}\left\{{ }^{1} \mathrm{H}\right\}$ NMR $\left(150 \mathrm{MHz}\right.$, DMSO- $\left.d_{6}\right)$ spectra of compound $\mathbf{4 z}$ 


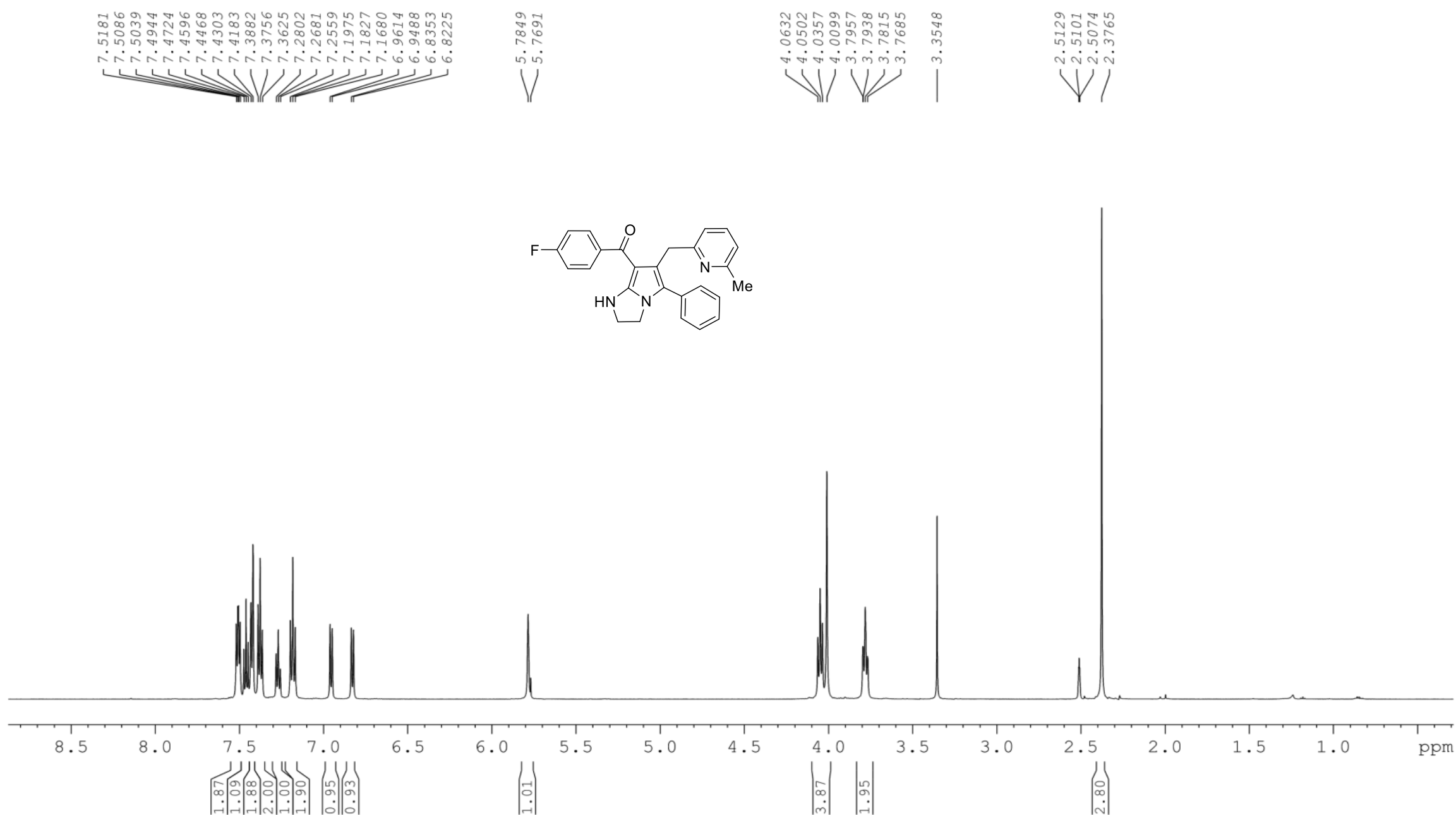

Figure S67. ${ }^{1} \mathrm{H}$ NMR $\left(600 \mathrm{MHz}, \mathrm{DMSO}-d_{6}\right)$ spectra of compound $4 \mathbf{a}^{\prime}$ 


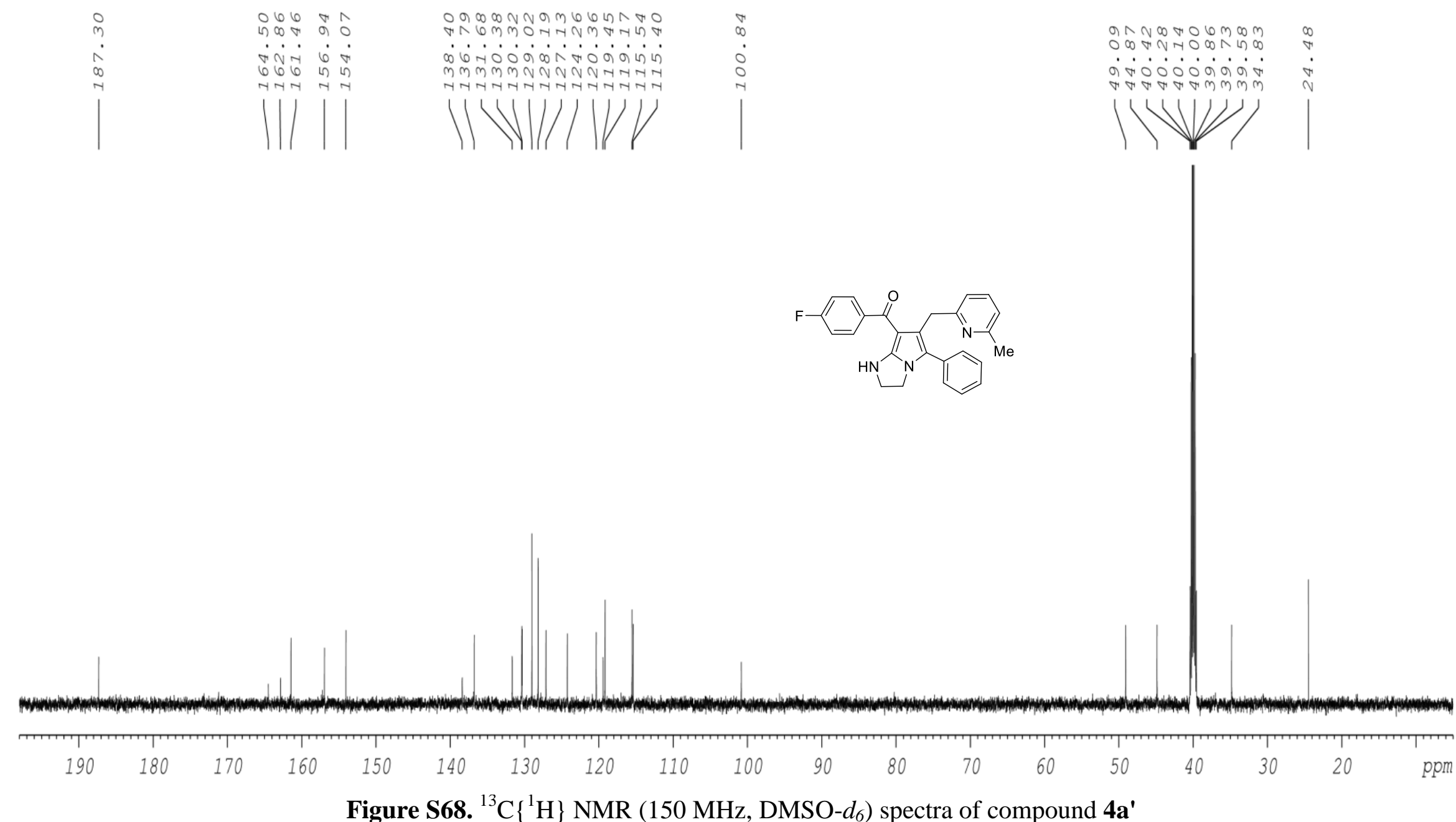




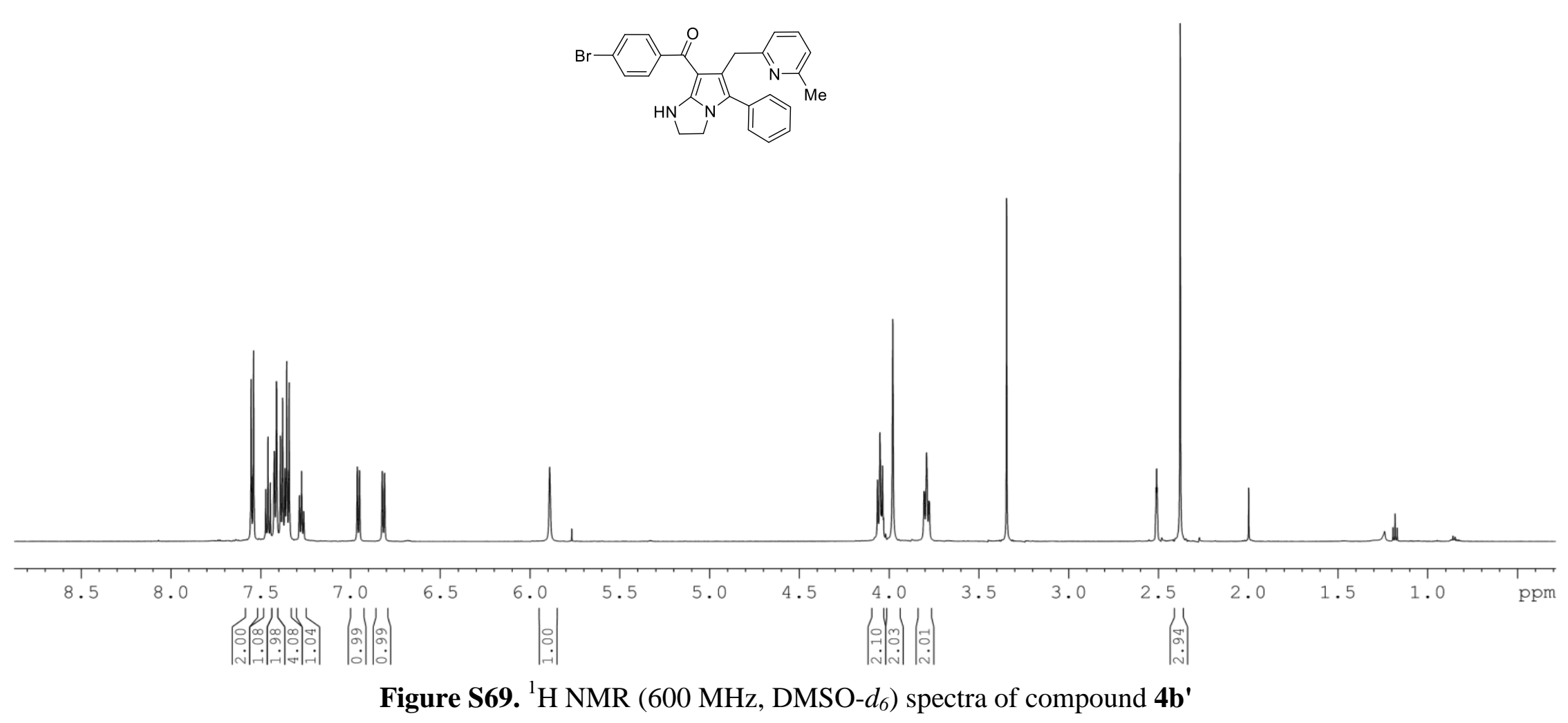

S74 


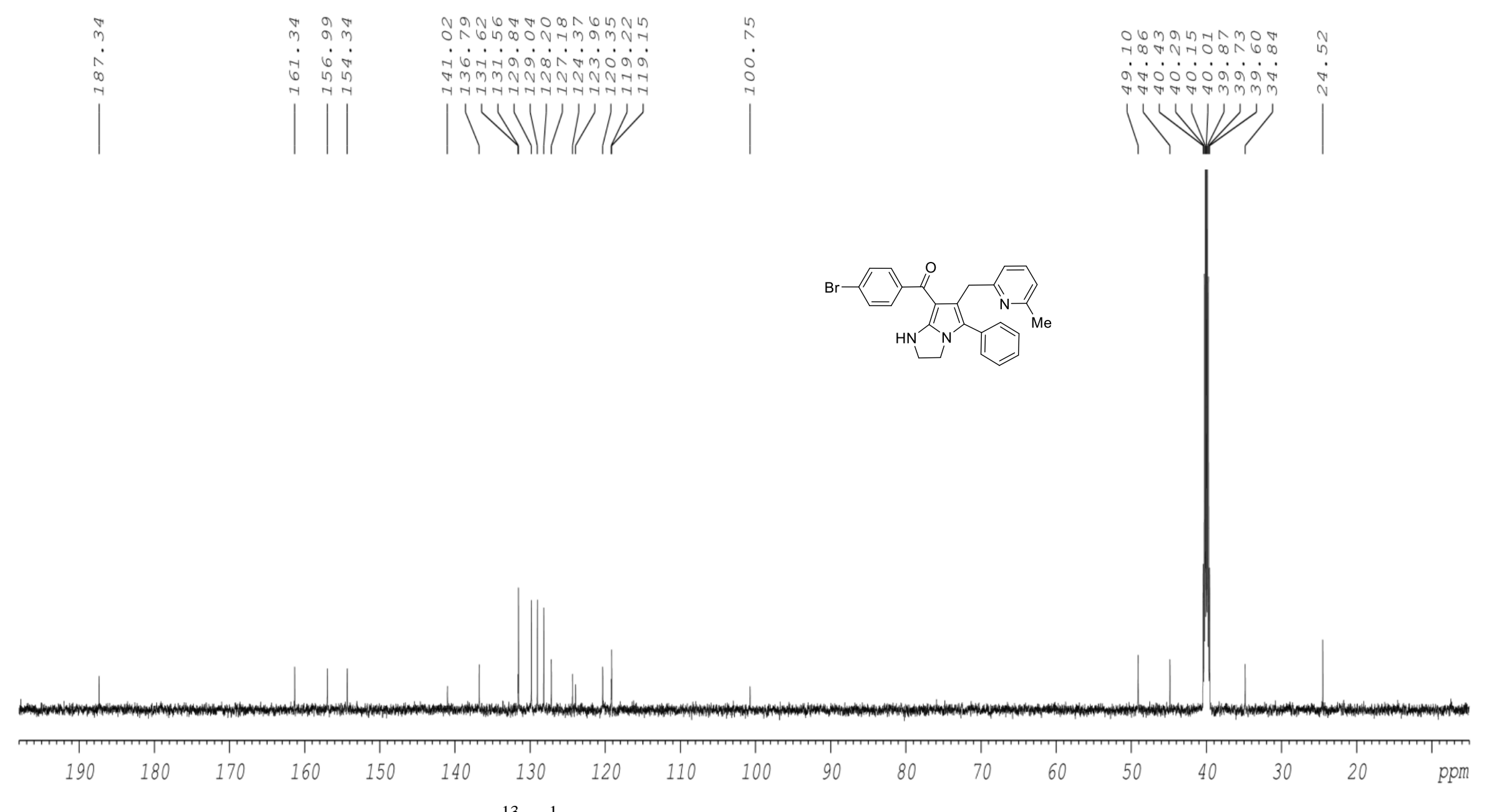

Figure S70. ${ }^{13} \mathrm{C}\left\{{ }^{1} \mathrm{H}\right\}$ NMR (150 MHz, DMSO- $\left.d_{6}\right)$ spectra of compound $4 \mathbf{b}^{\mathbf{}}$ 

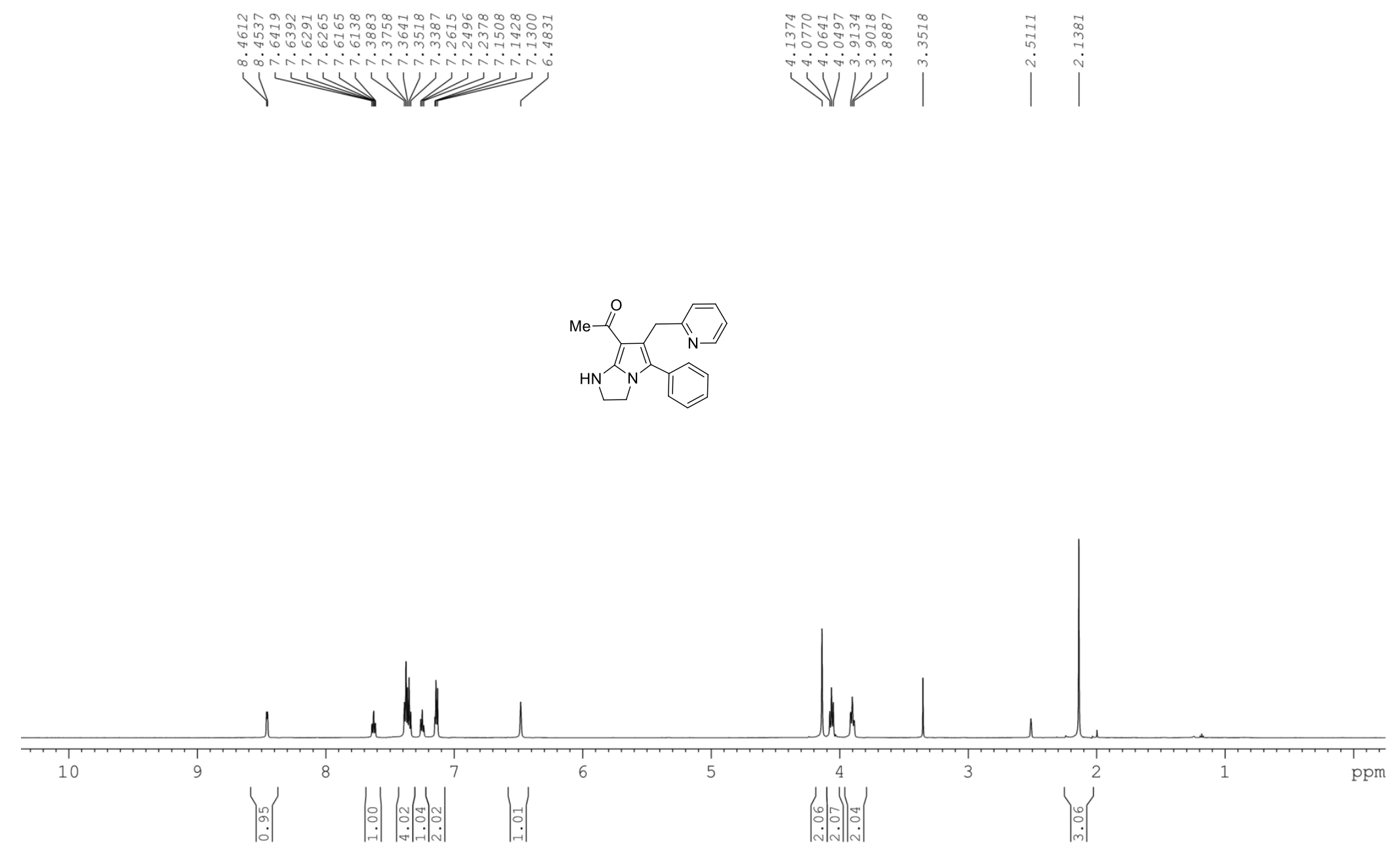

Figure S71. ${ }^{1} \mathrm{H}$ NMR (600 MHz, DMSO- $\left.d_{6}\right)$ spectra of compound 5a 


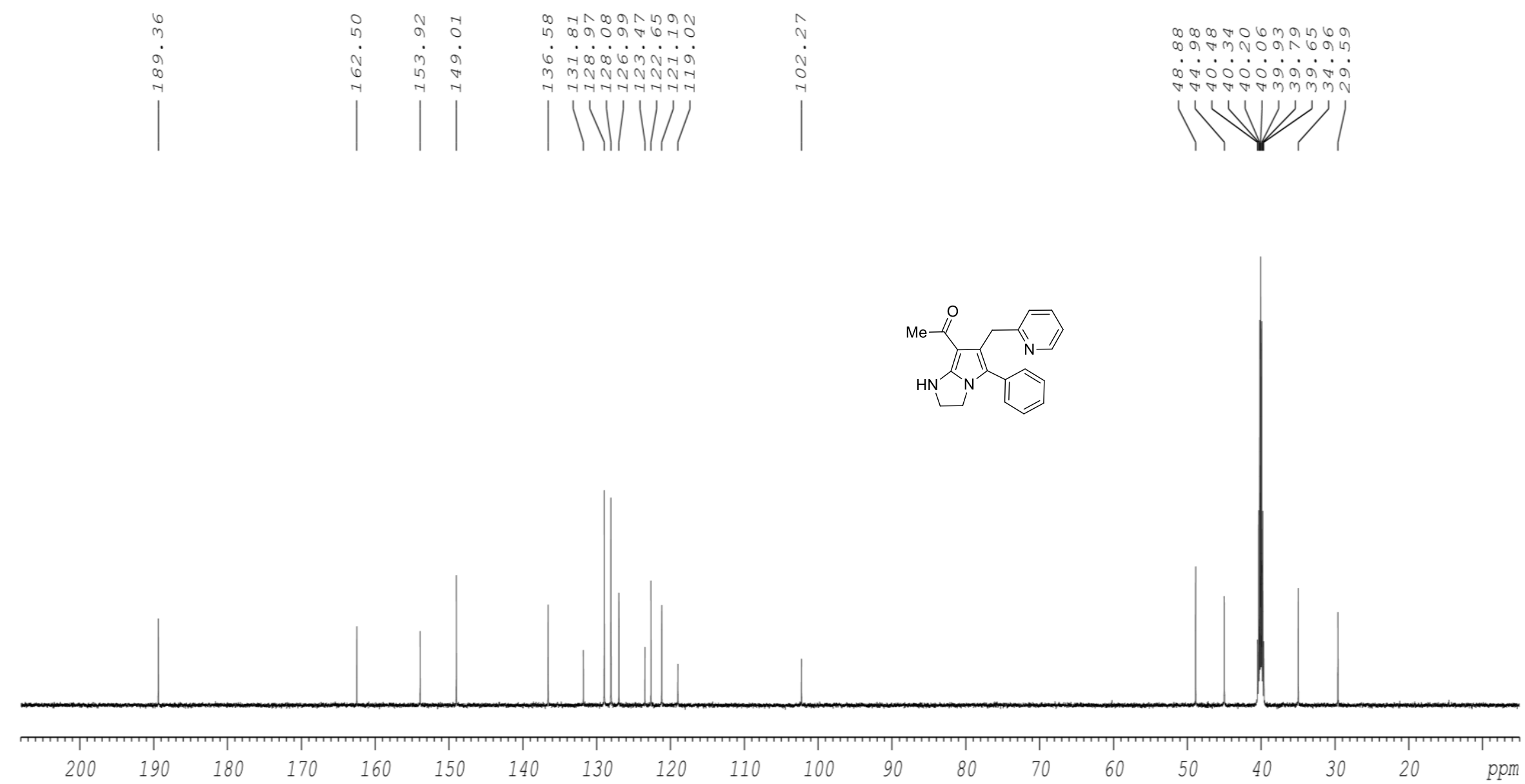

Figure S72. ${ }^{13} \mathrm{C}\left\{{ }^{1} \mathrm{H}\right\}$ NMR $\left(150 \mathrm{MHz}\right.$, DMSO- $\left.d_{6}\right)$ spectra of compound 5a 

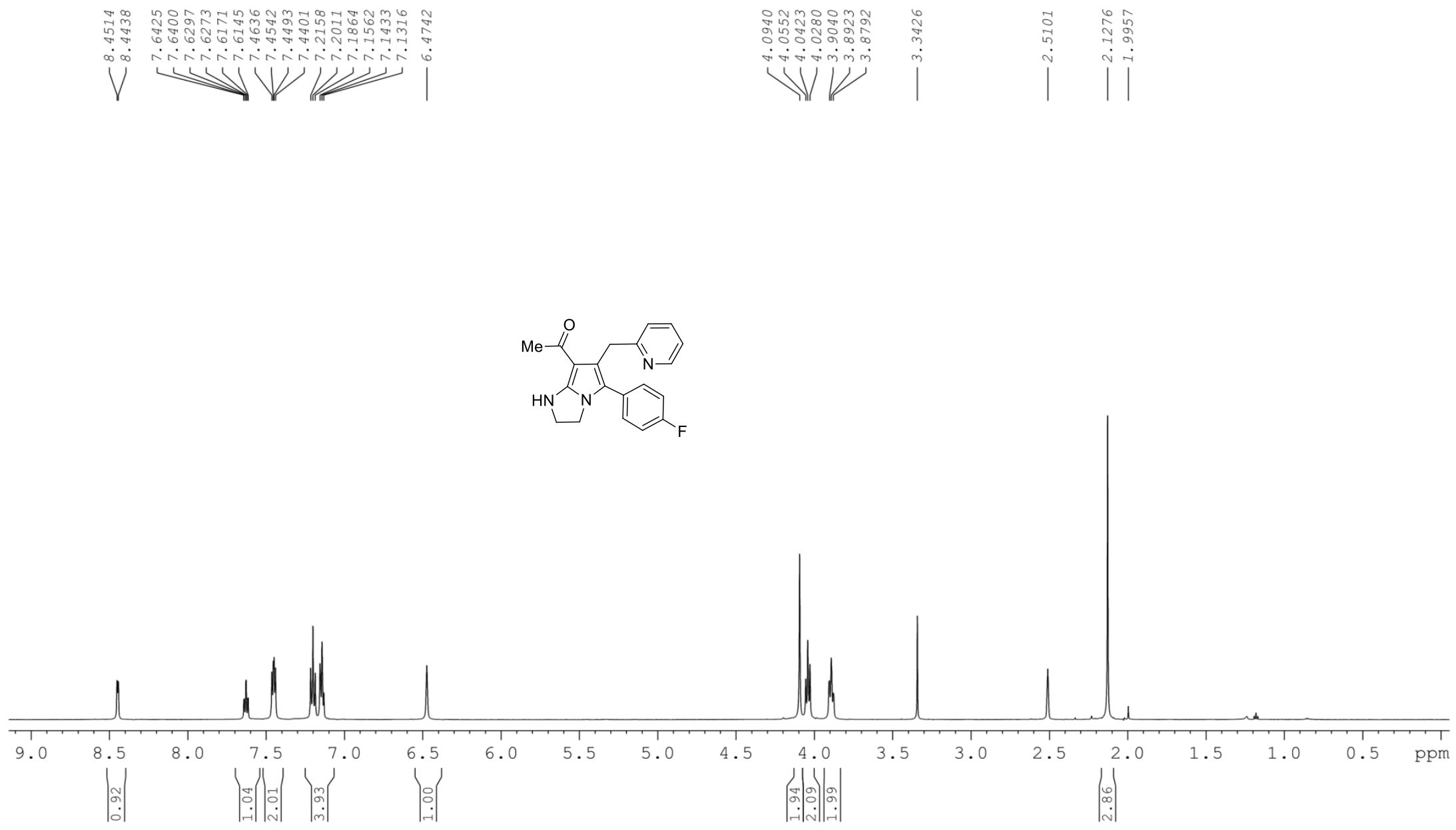

Figure S73. ${ }^{1} \mathrm{H}$ NMR (600 MHz, DMSO- $\left.d_{6}\right)$ spectra of compound $\mathbf{5 b}$ 


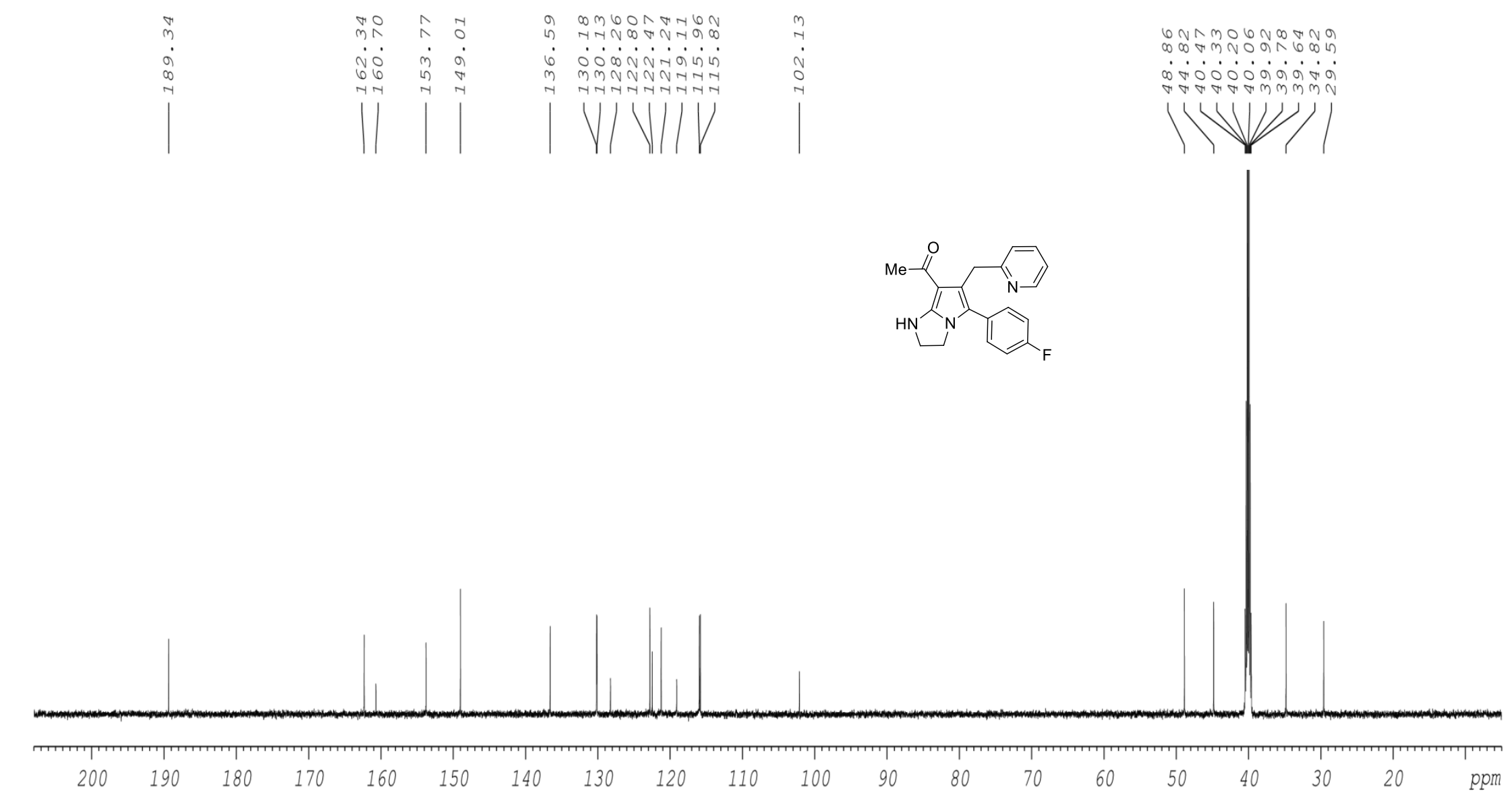

Figure S74. ${ }^{13} \mathrm{C}\left\{{ }^{1} \mathrm{H}\right\}$ NMR (150 MHz, DMSO- $\left.d_{6}\right)$ spectra of compound $\mathbf{5 b}$ 

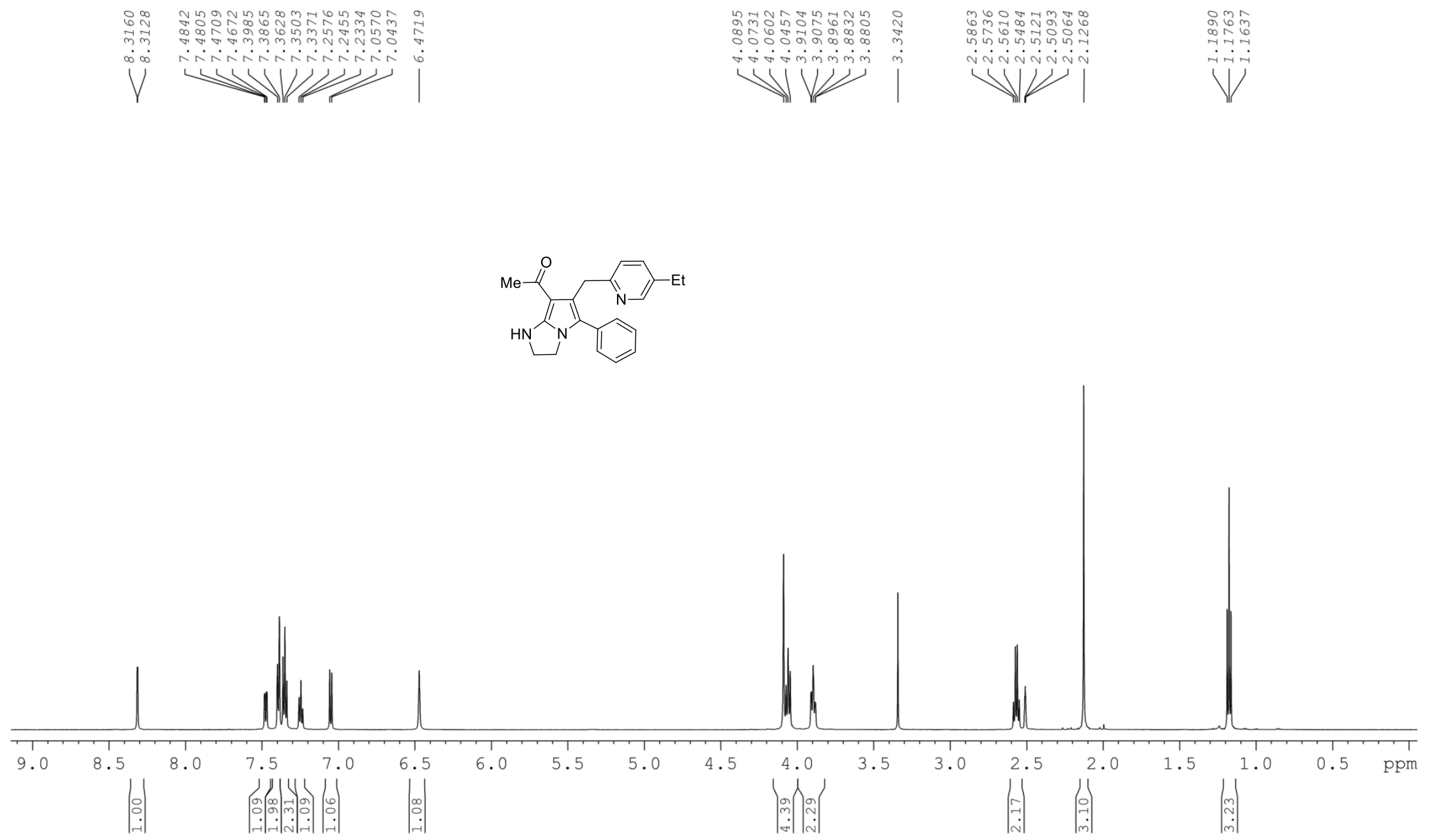

Figure S75. ${ }^{1} \mathrm{H}$ NMR $\left(600 \mathrm{MHz}, \mathrm{DMSO}-d_{6}\right)$ spectra of compound 5c 


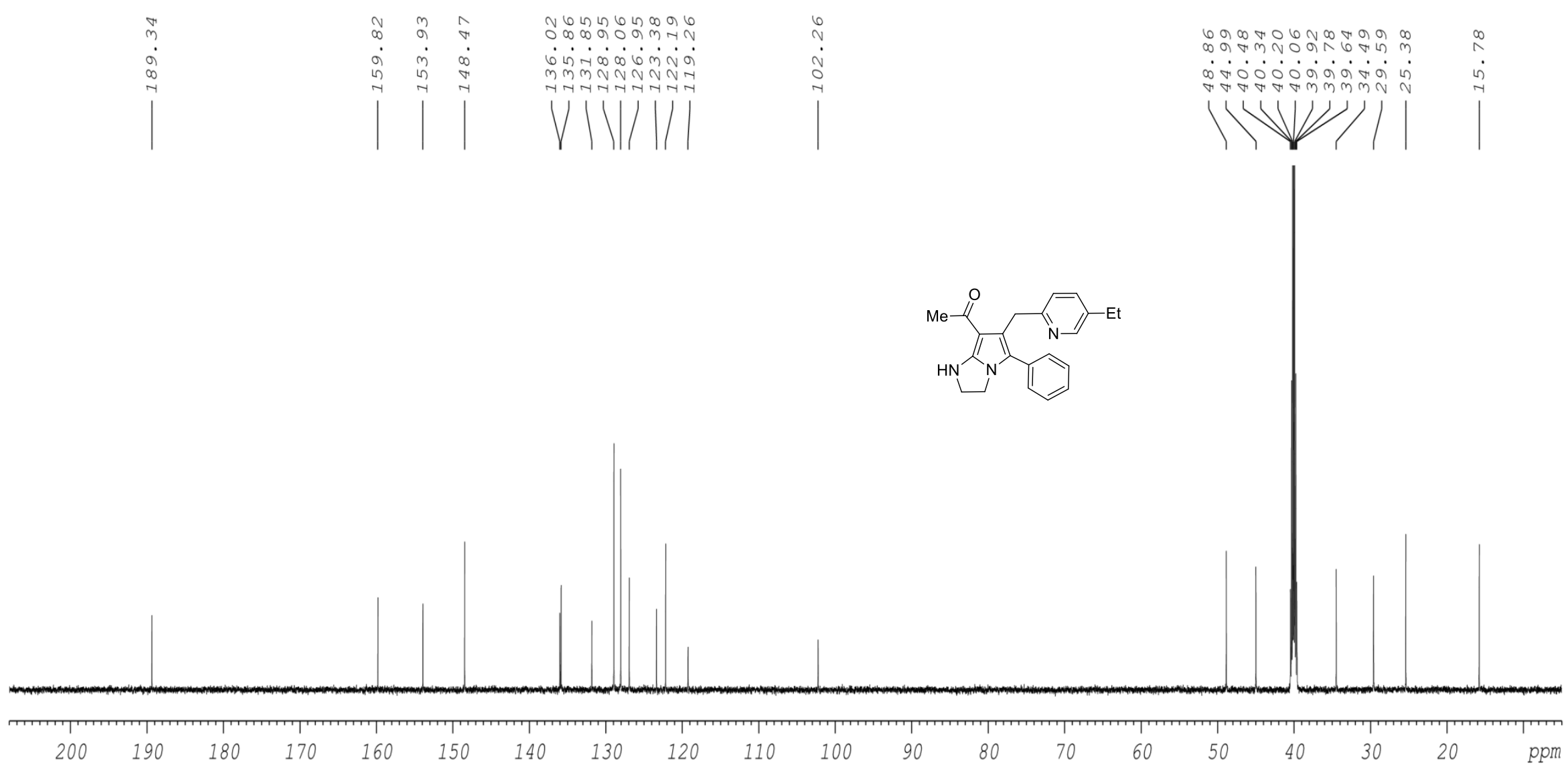

Figure S76. ${ }^{13} \mathrm{C}\left\{{ }^{1} \mathrm{H}\right\}$ NMR $\left(150 \mathrm{MHz}, \mathrm{DMSO}-d_{6}\right)$ spectra of compound 5c 

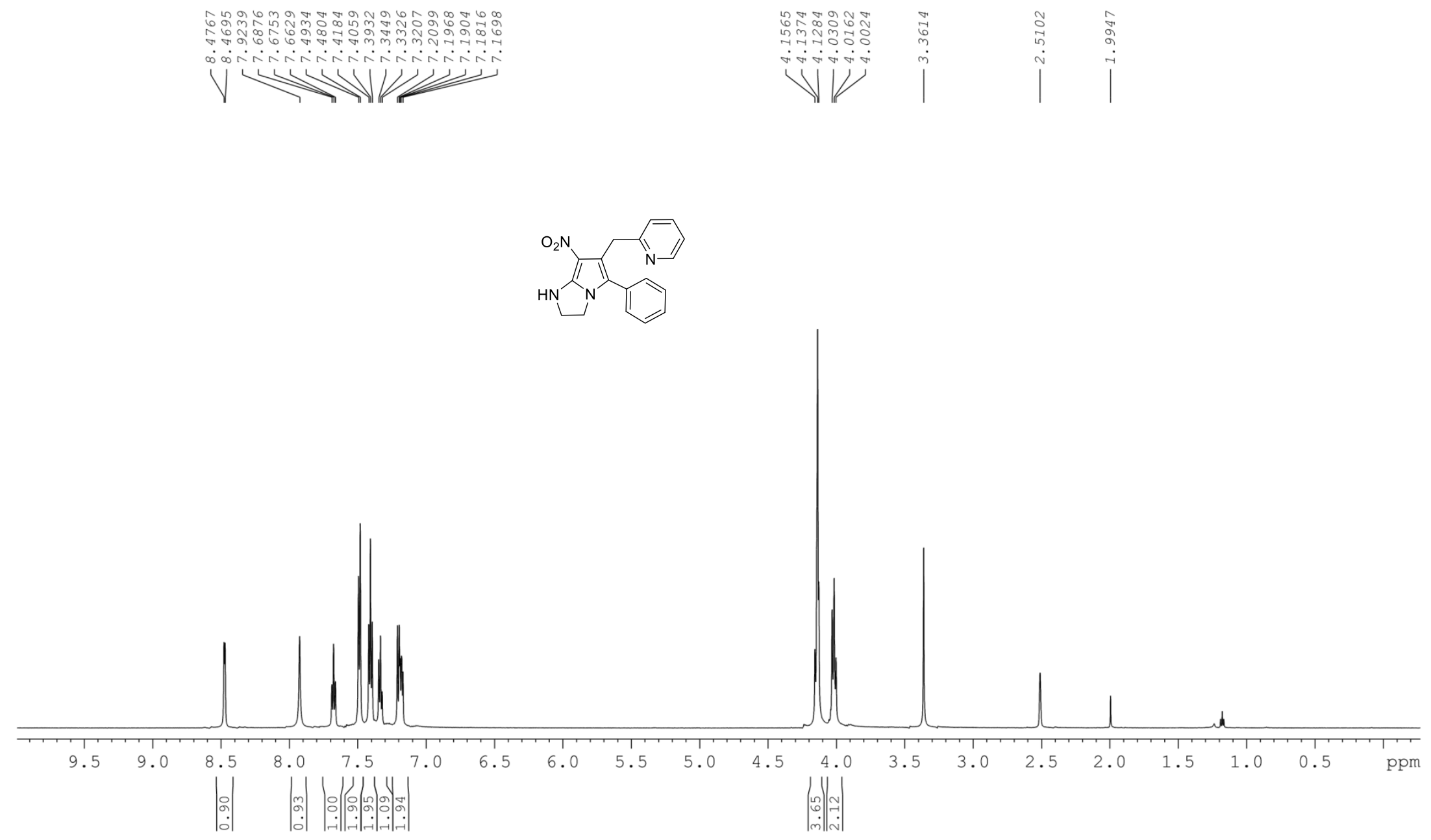

Figure S77. ${ }^{1} \mathrm{H}$ NMR $\left(600 \mathrm{MHz}, \mathrm{DMSO}-d_{6}\right)$ spectra of compound $\mathbf{5 d}$ 


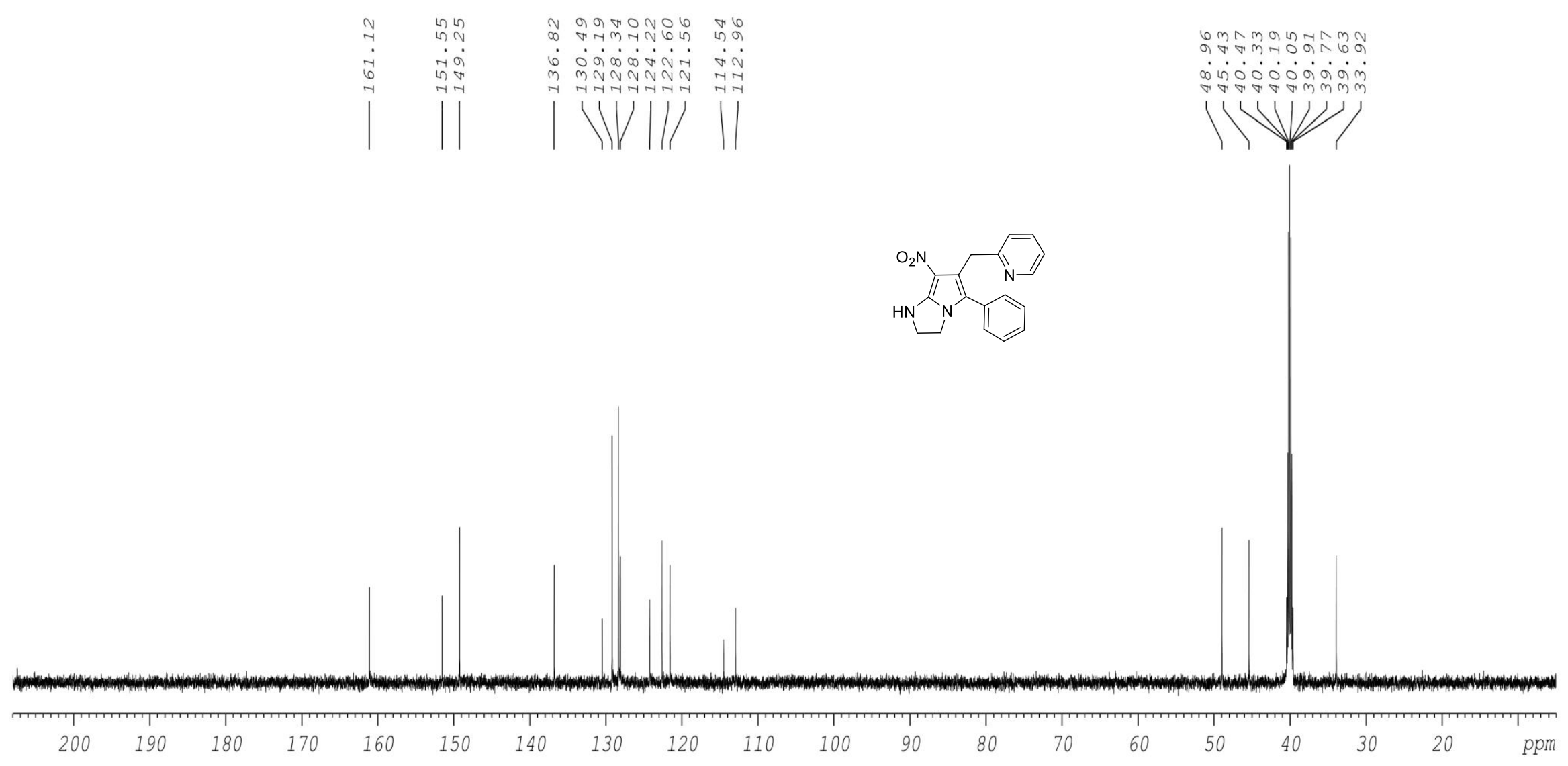

Figure S78. ${ }^{13} \mathrm{C}\left\{{ }^{1} \mathrm{H}\right\}$ NMR $\left(150 \mathrm{MHz}, \mathrm{DMSO}-d_{6}\right)$ spectra of compound 5d 


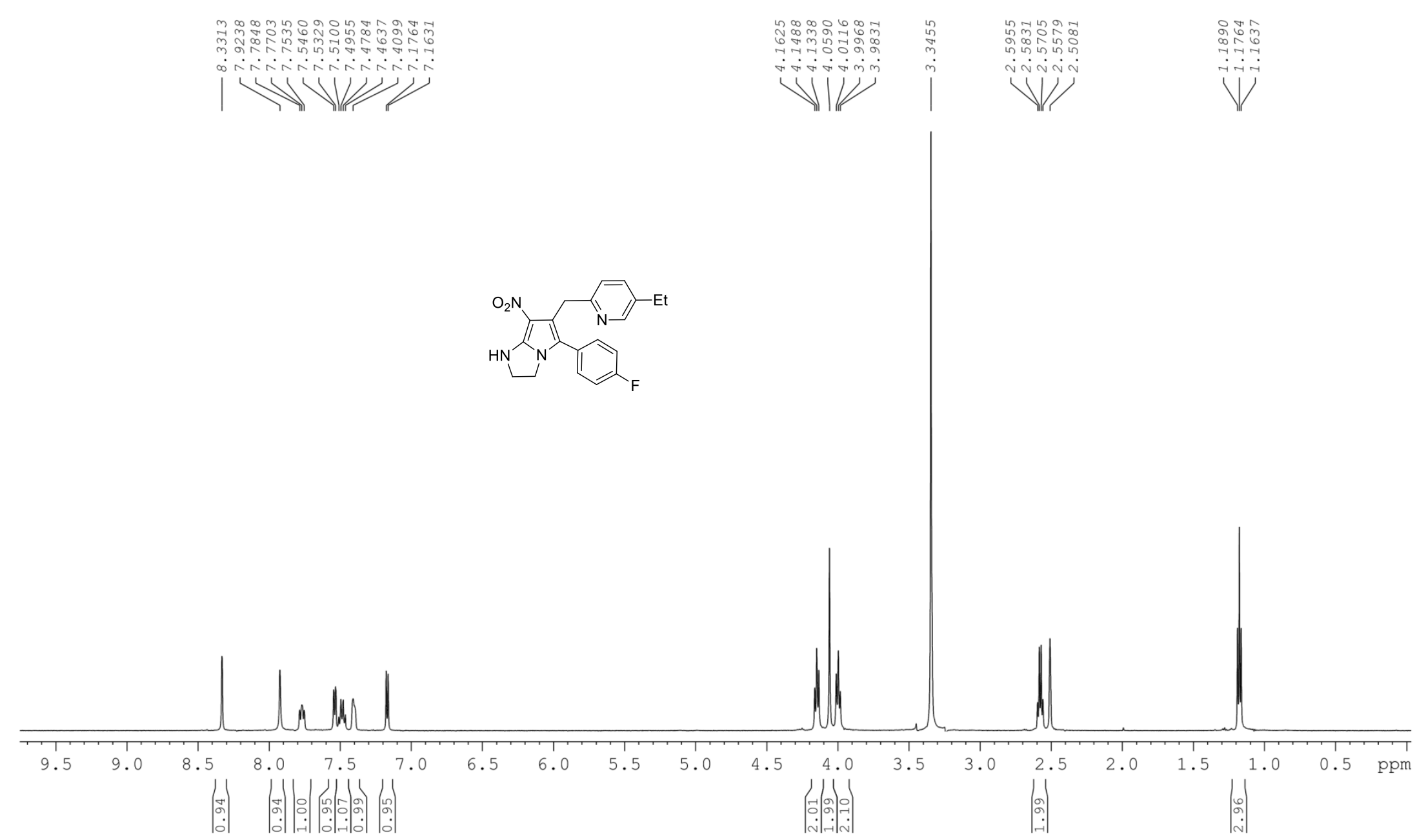

Figure S79. ${ }^{1} \mathrm{H}$ NMR (600 MHz, DMSO- $\left.d_{6}\right)$ spectra of compound 5e 

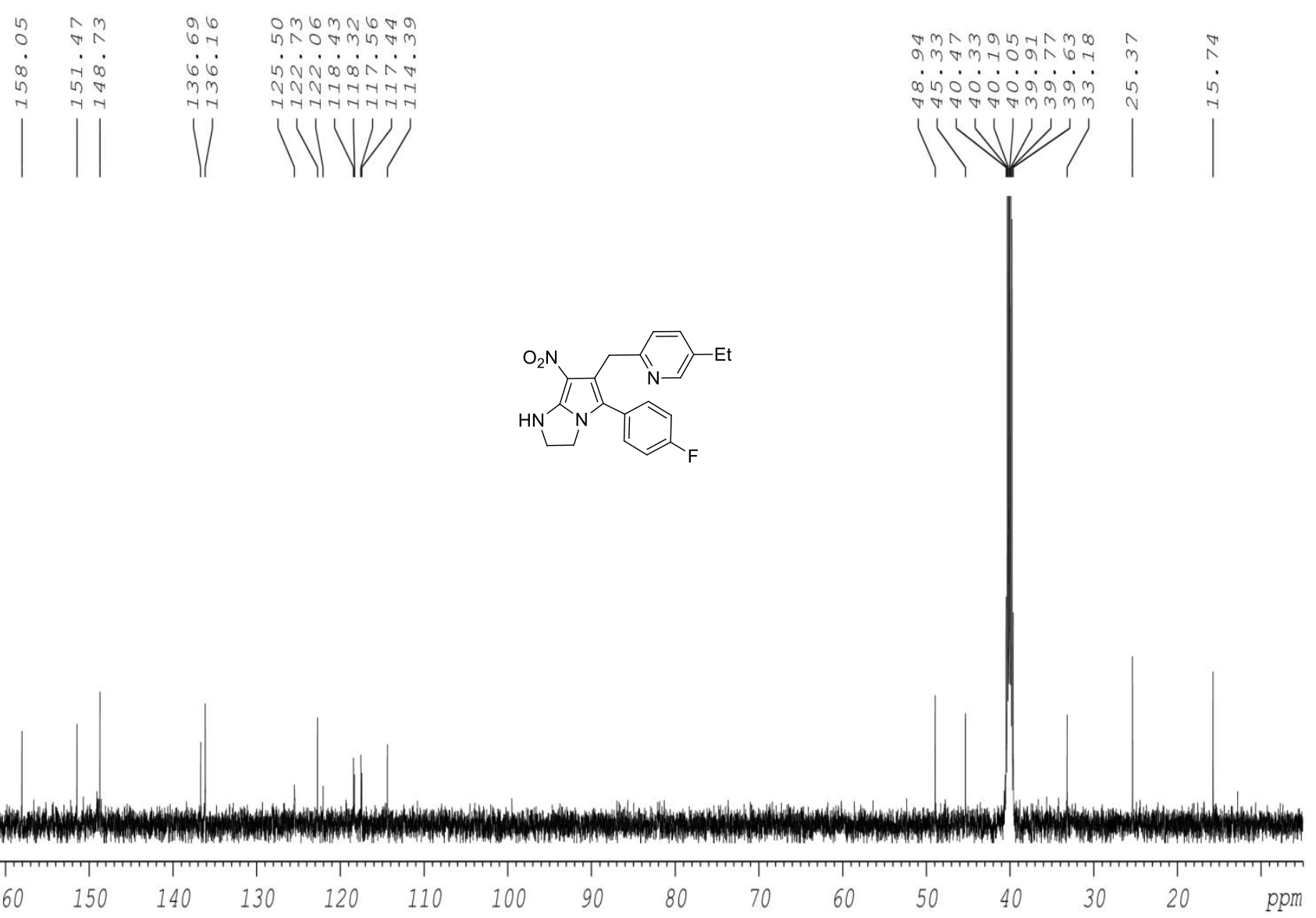

Figure S80. ${ }^{13} \mathrm{C}\left\{{ }^{1} \mathrm{H}\right\}$ NMR $\left(150 \mathrm{MHz}, \mathrm{DMSO}-d_{6}\right)$ spectra of compound $5 \mathbf{e}$ 

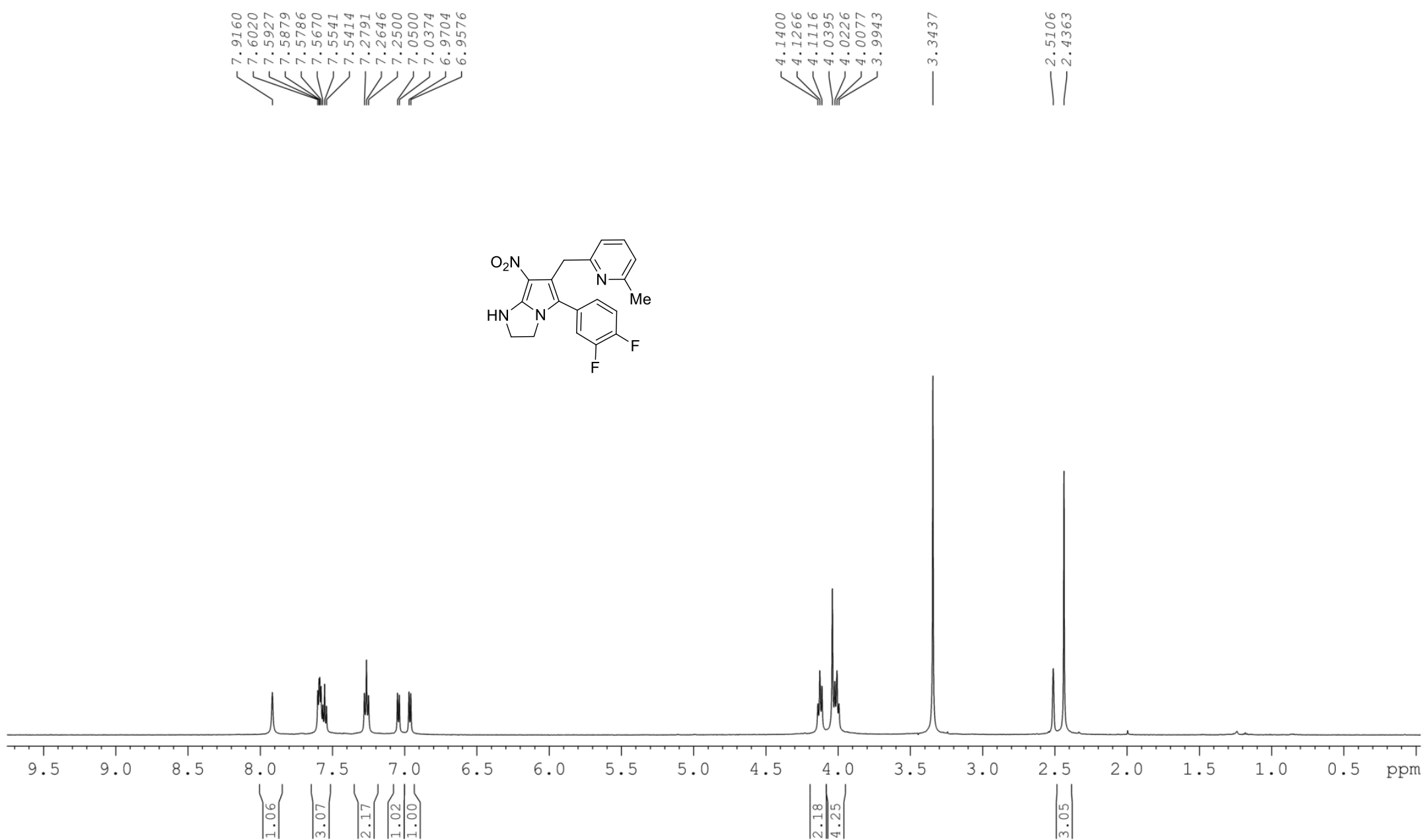

Figure S81. ${ }^{1} \mathrm{H}$ NMR $\left(600 \mathrm{MHz}, \mathrm{DMSO}-d_{6}\right)$ spectra of compound $\mathbf{5 f}$ 

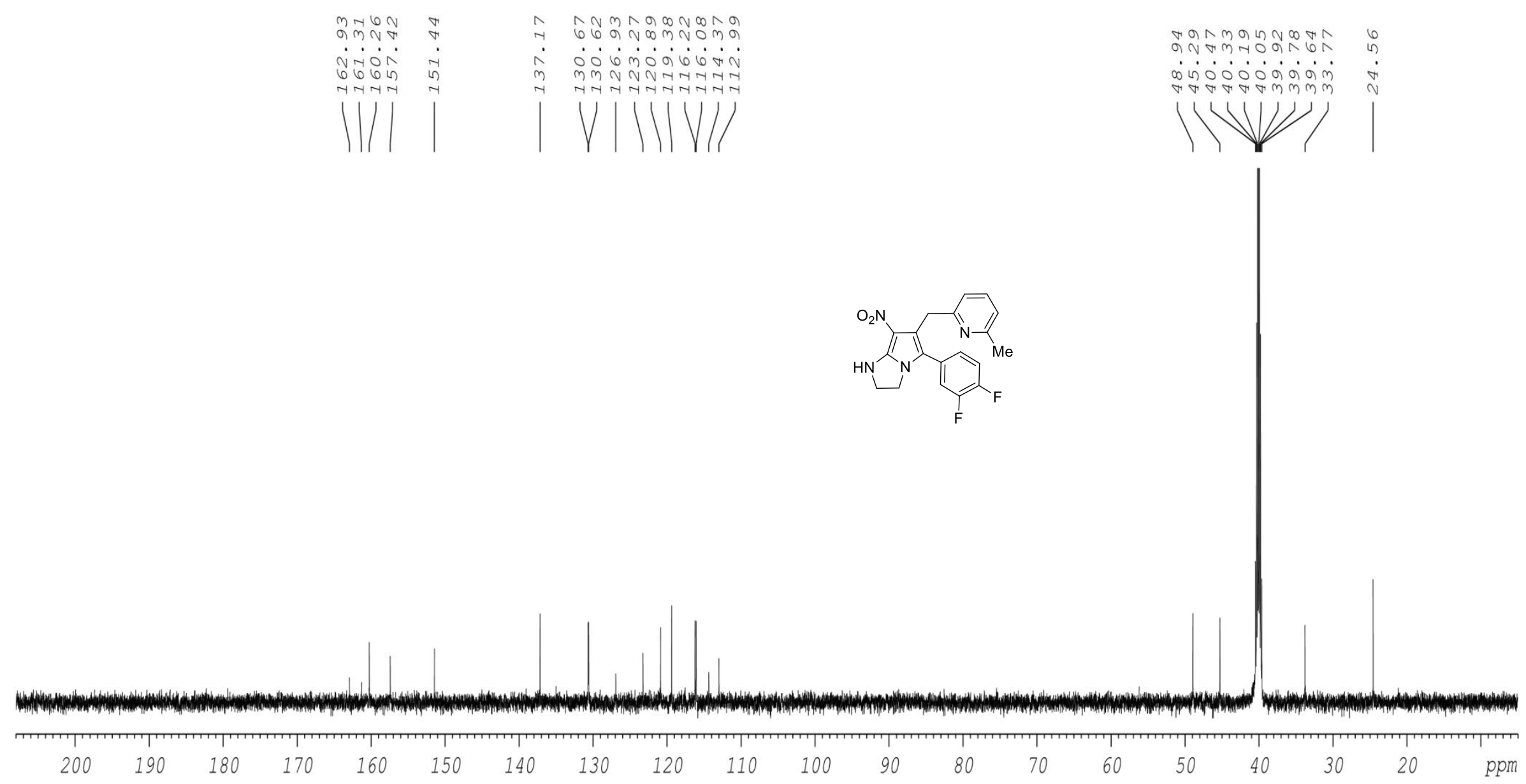

Figure S82. ${ }^{13} \mathrm{C}\left\{{ }^{1} \mathrm{H}\right\}$ NMR (150 MHz, DMSO- $\left.d_{6}\right)$ spectra of compound $\mathbf{5 f}$ 


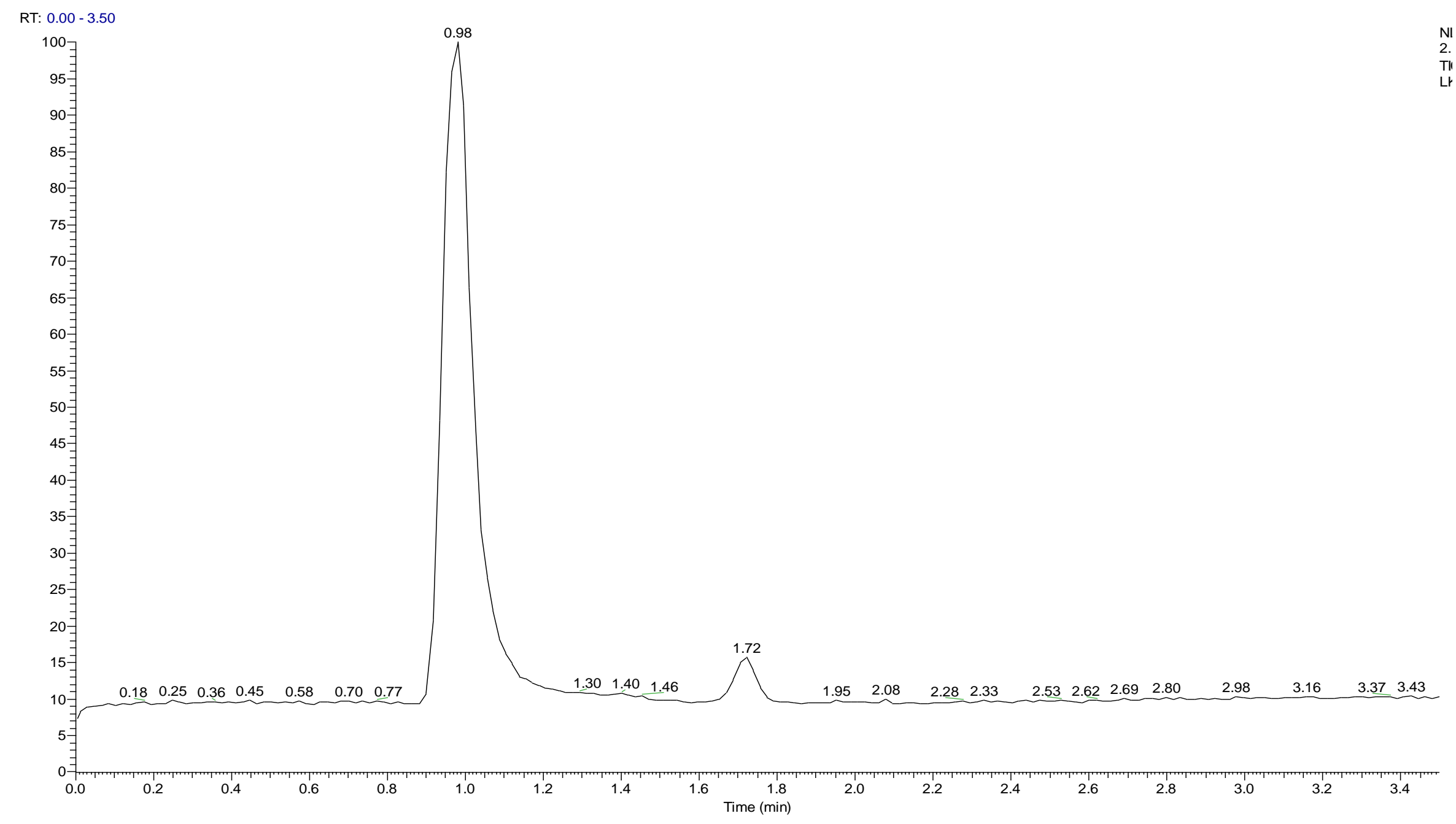

Figure S83. HPLC of the reaction mixture 
LKC \#53 RT: 0.94 AV: 1 NL: $8.41 E 6$

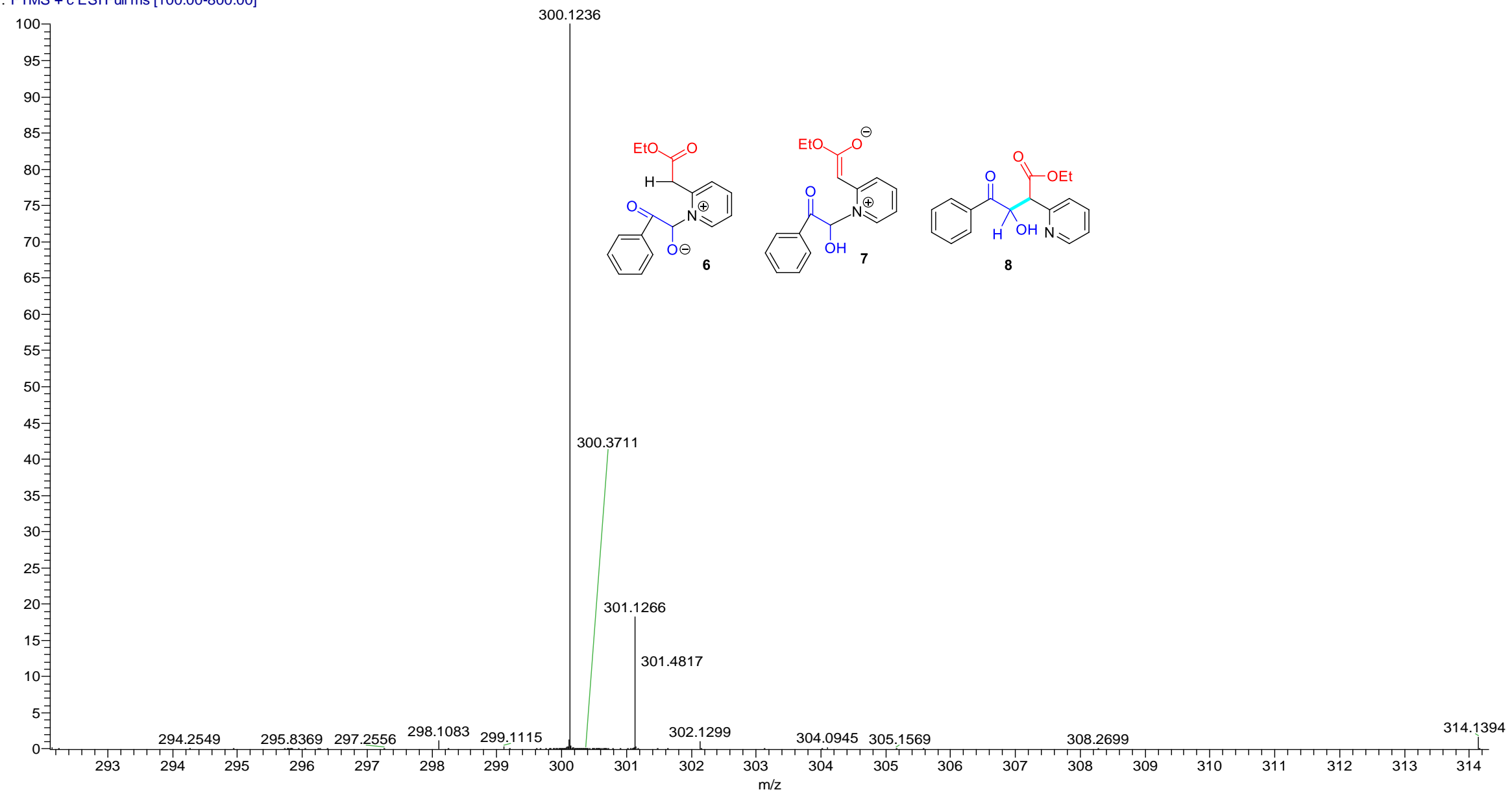

Figure S84. HRMS of intermediate 6/7/8 


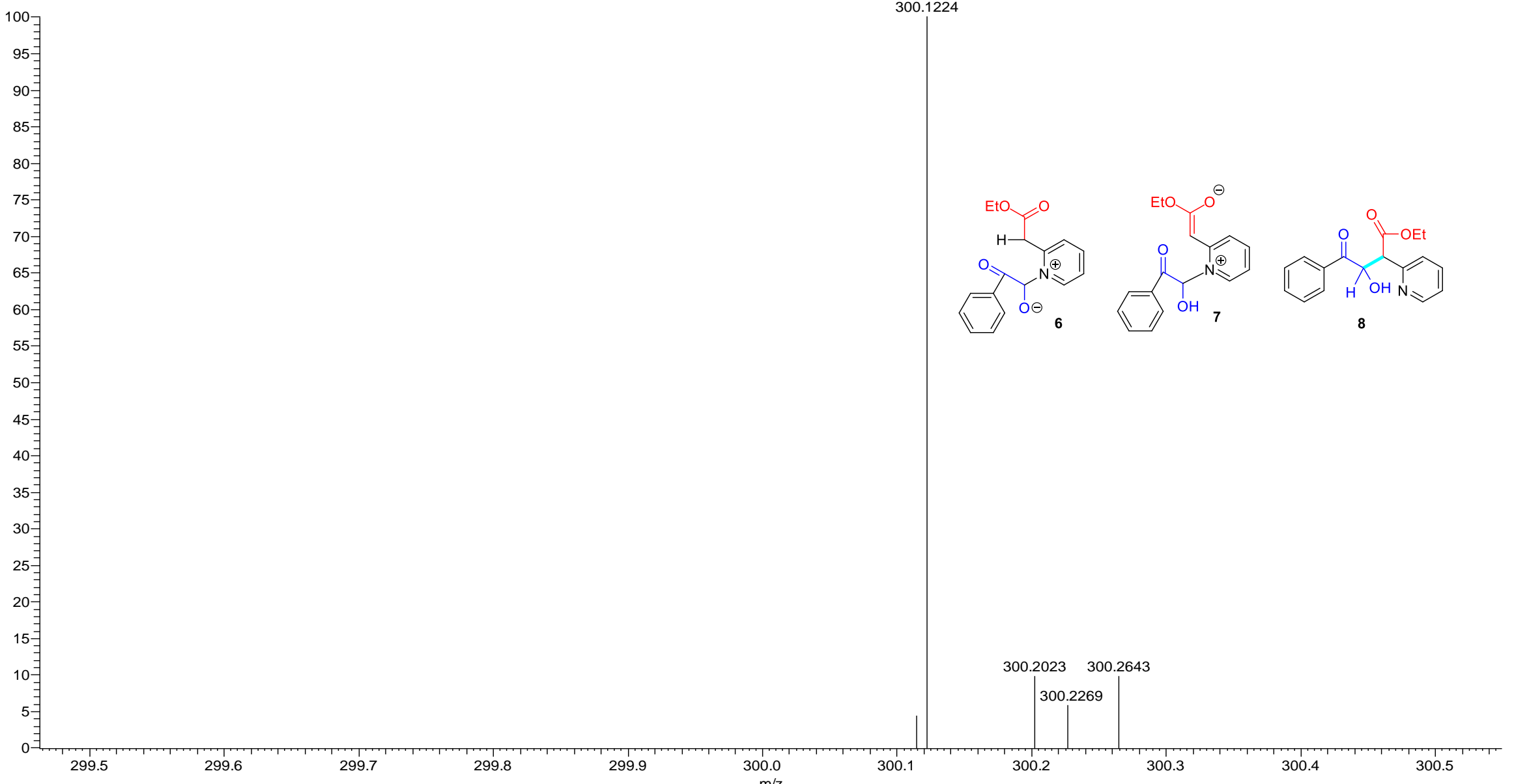

Figure S85. HRMS of intermediate 6/7/8 


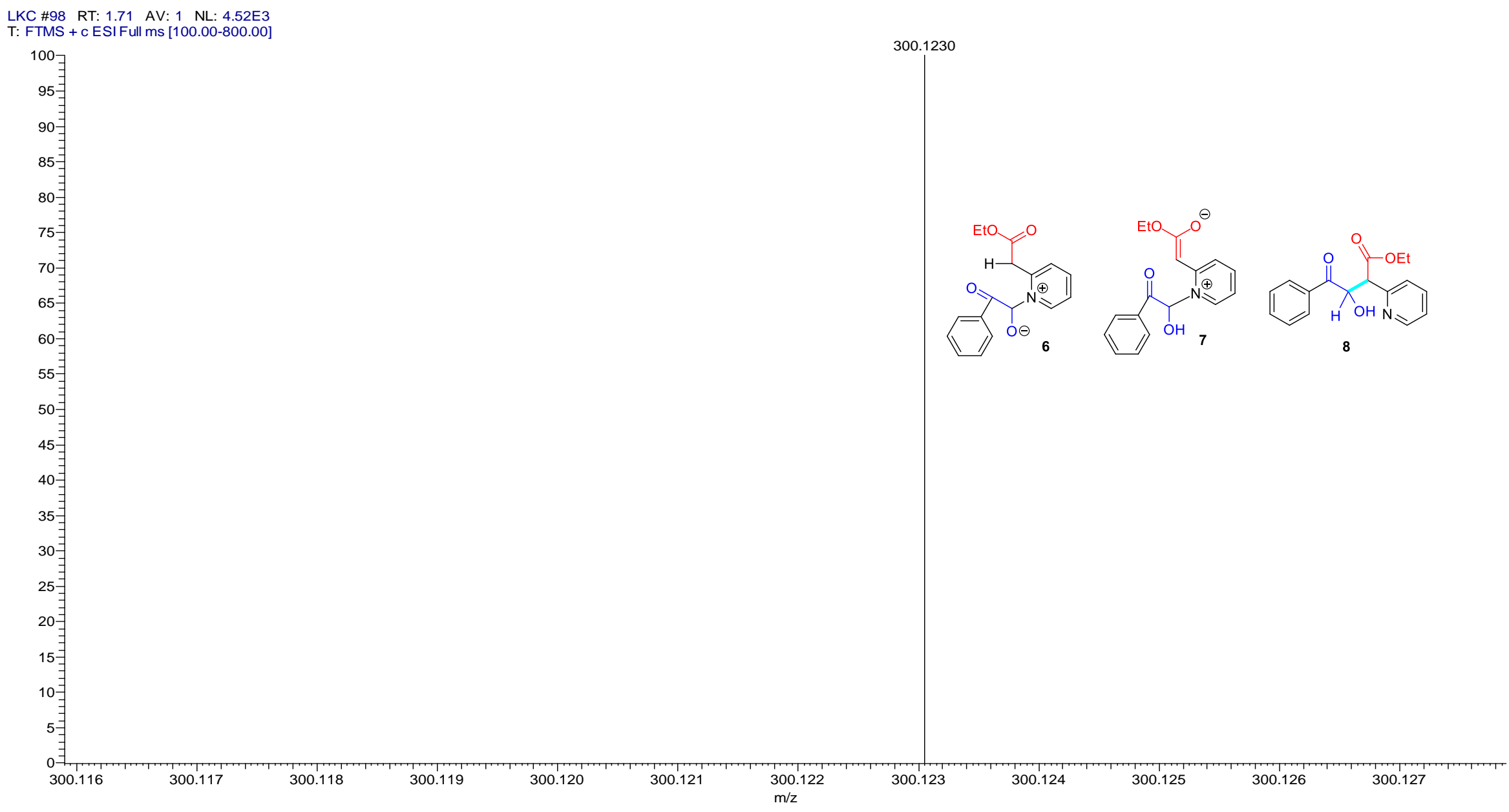

Figure S86. HRMS of intermediate 6/7/8 


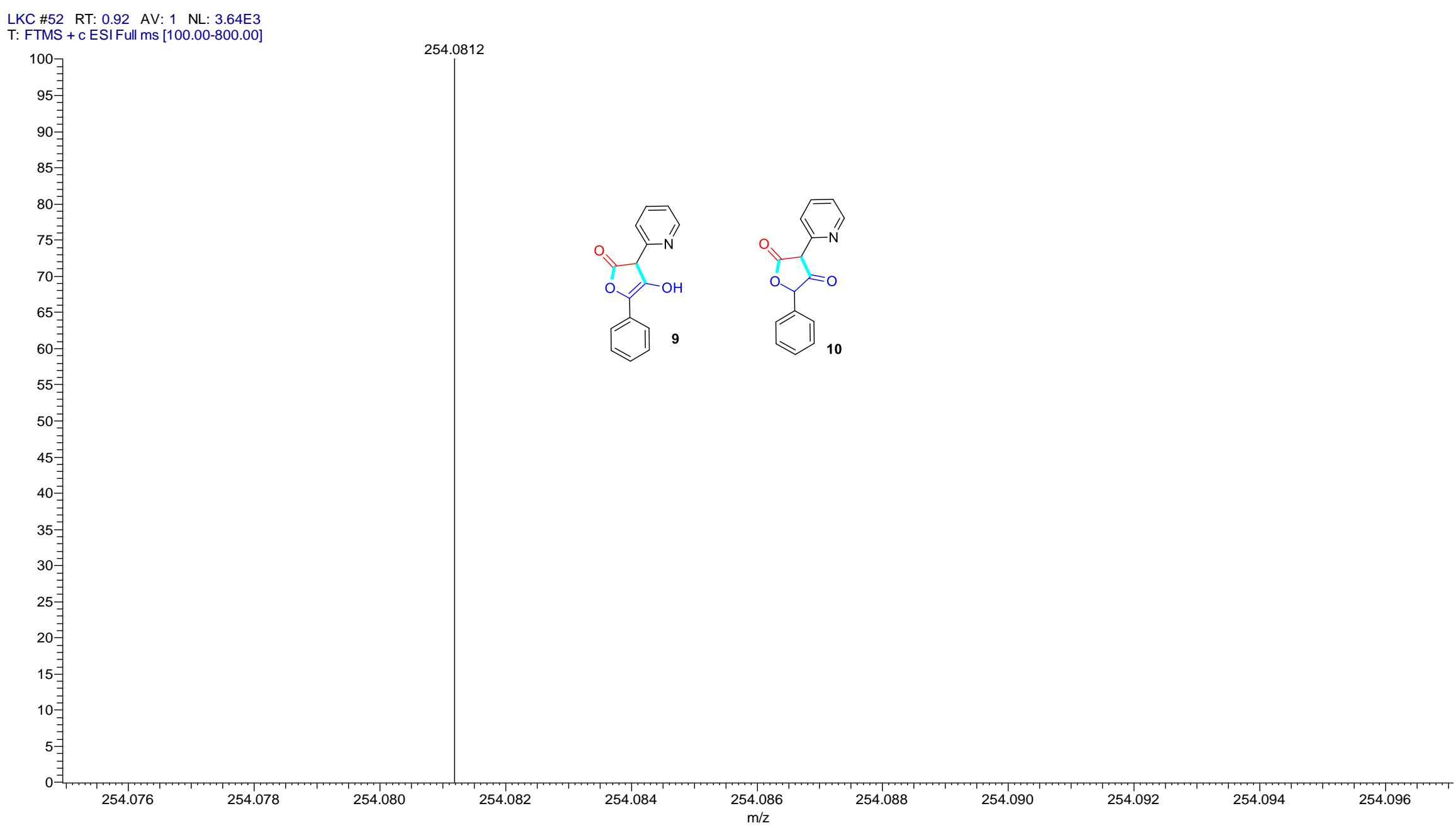

Figure S87. HRMS of intermediate 9/10 
LKC \#54 RT: 0.95 AV: $1 \quad$ NL: 4.78E3

+ c ESI Full ms [100.00-800.00]

100

$95 \exists$

90 글

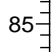

80

70

$65=$

$60=$

55

$50 \exists$

$45=$

40

35

30

25 ㅋ.

20 ق

$15 \mathrm{\exists}$

10

$5 \exists$

254.0819

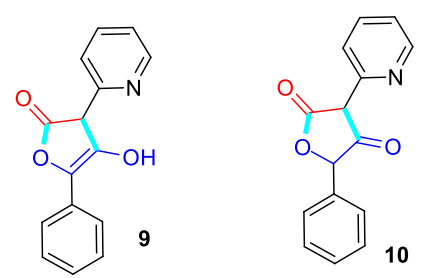

10

Figure S88. HRMS of intermediate 9/10 


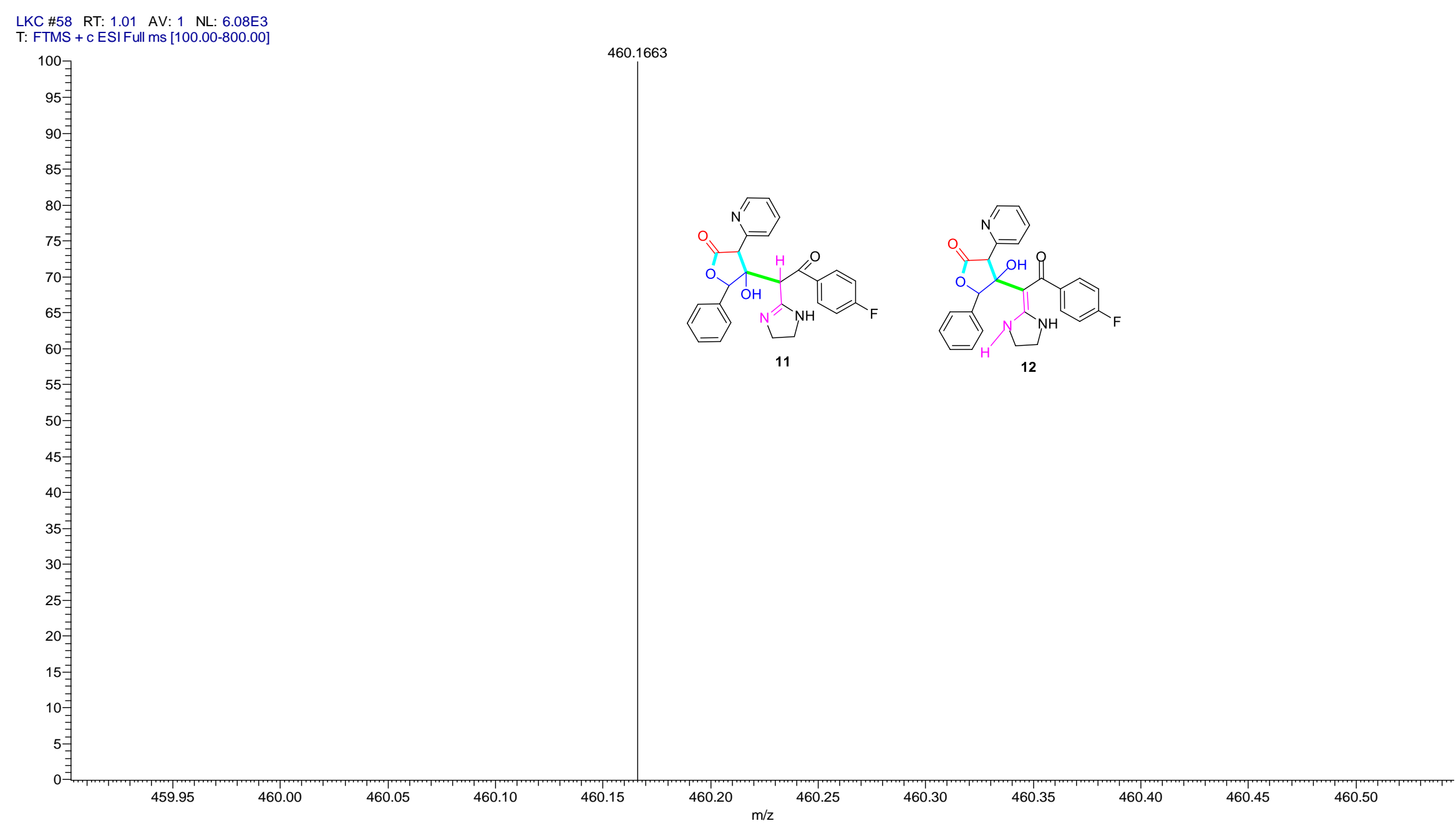

Figure S89. HRMS of intermediate $\mathbf{1 1}$ or $\mathbf{1 2}$ 


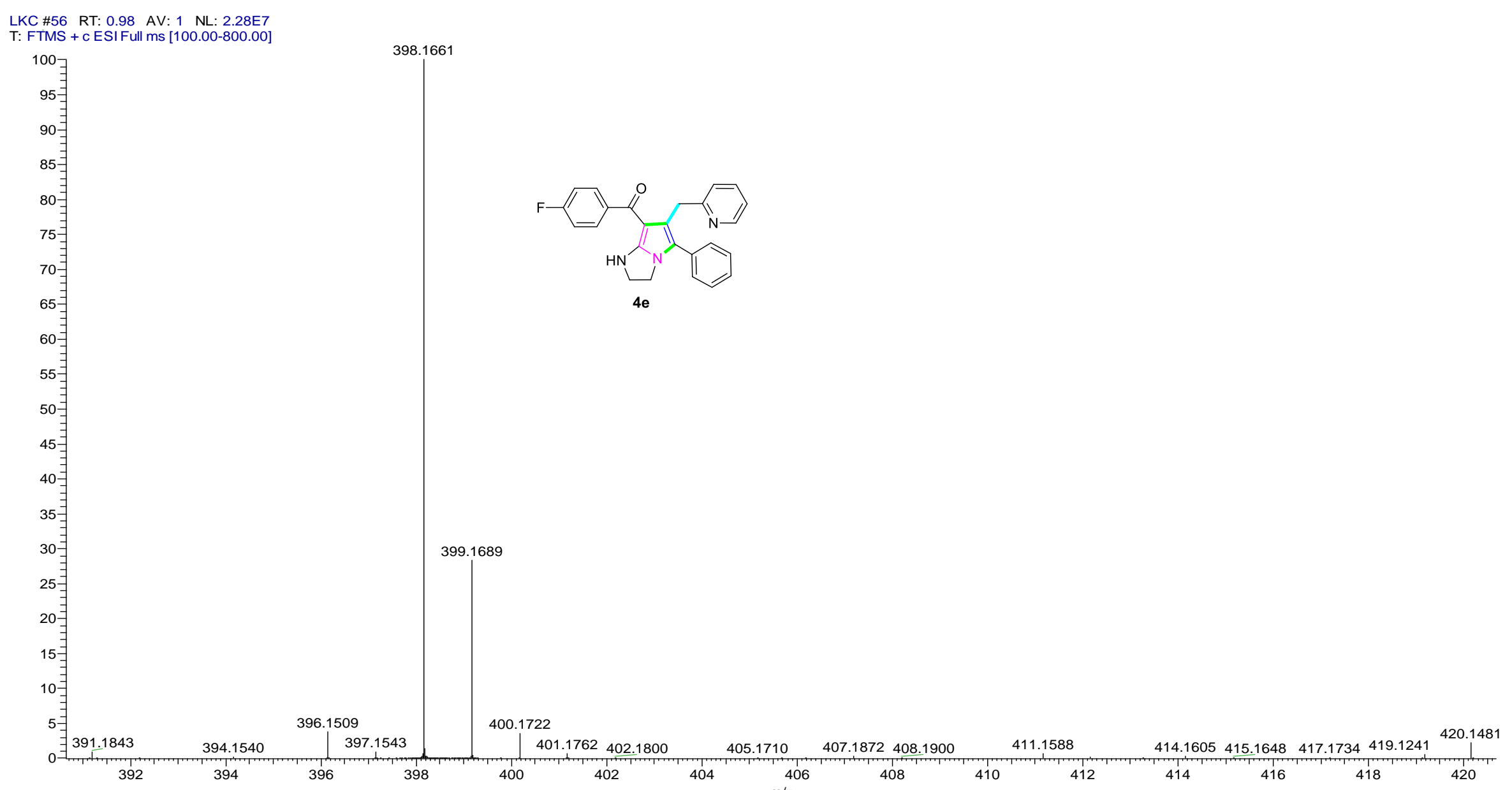

Figure S90. HRMS of intermediate 4e 


\section{References and Notes}

1. CCDC1920258 contain the supplementary crystallographic data for compound 4a. These data can be obtained free of charge from The Cambridge Crystallographic Data Center via www.ccdc.cam.ac.uk/data request/cif 